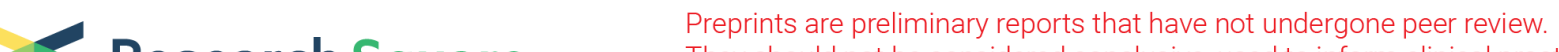 Research Square They should not be considered conclusive, used to inform clinical practice, or referenced by the media as validated information.
}

\section{Multi-source and Multi-fault Condition Monitoring Based on Parallel Factor Analysis and Sequential Probability Ratio Test}

\section{Liu Yang}

No. 206, Guanggu First Road, Jiangxia District

Hanxin Chen ( $\square$ pg01074075@163.com )

Wuhan Institute of Technology

\section{Yao Ke}

No. 206, Guanggu First Road, Jiangxia District

Menglong Li

No. 206, Guanggu First Road, Jiangxia District

\section{Lang Huang}

No. 206, Guanggu First Road, Jiangxia District

\section{Yuzhuo Miao}

No. 206, Guanggu First Road, Jiangxia District

\section{Research}

Keywords: PARAFAC, Tensor decomposition, SPRT, Fault diagnosis, Centrifugal pump

Posted Date: March 15th, 2021

DOI: https://doi.org/10.21203/rs.3.rs-282746/v1

License: () (1) This work is licensed under a Creative Commons Attribution 4.0 International License. Read Full License

Version of Record: A version of this preprint was published at EURASIP Journal on Advances in Signal Processing on July 13th, 2021. See the published version at https://doi.org/10.1186/s13634-021-00730W. 


\title{
Multi-source and multi-fault condition monitoring based on parallel factor analysis and sequential probability ratio test
}

\author{
Liu Yang ${ }^{1, \mathrm{a}}$, Hanxin Chen ${ }^{1,2, b^{* *}}\left(\right.$ Corresponding Author), Yao Ke ${ }^{1, \mathrm{c}}$, Menglong $\mathrm{Li}^{1, \mathrm{~d}}$, Lang Huang ${ }^{1, \mathrm{e}}$, Yuzhuo Miao ${ }^{1, \mathrm{f}}$ \\ ${ }^{1}$ No. 206, Guanggu First Road, Jiangxia District, Wuhan, Hubei, 430205,P.R. China \\ ${ }^{2}$ Hubei Provincial Key Laboratory of Chemical Equipment, Intensification and Intrinsic Safety, Wuhan Institute of \\ Technology, Wuhan; 430073, China \\ a201615004@stu.wit.edu.cn, bpg01074075@163.com(Corresponding Author), '13628631803@163.com,

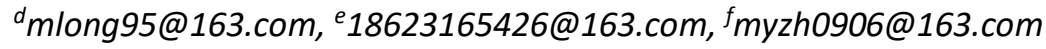

\begin{abstract}
The monitoring of mechanical equipment systems contains an increasing number of complex content, expanding from traditional time and frequency information to three-dimensional data of the time, space and frequency information, and even higher-dimensional data containing subjects , experimental conditions. For high-dimensional data analysis, traditional decomposition methods such as Hilbert Transform, Fast Fourier Transformation and Gabor transformation not only lose the integrity of the data, but also increase the amount of calculation and introduce a lot of redundant information. The phenomenon of feature coupling, aliasing and redundancy between the mechanical multi-source data signals will cause the inaccuracy of the evaluation, diagnosis and prediction of industrial production operation status. The analysis of the three-way tensor composed of channel, frequency and time is called Parallel Factor Analysis (PARAFAC). The properties between the parallel factor analysis results and the input signals are studied through simulation experiments. Parallel factor analysis is used to decompose the third-order tensor composed of channel-time-frequency after continuous wavelet transformation of vibration signal into channel, time and frequency characteristics. Multi-scale parallel factor analysis successfully extracted nonlinear multi-dimensional dynamic fault characteristics by generating the spatial, spectral, time-domain signal loading value and three-dimensional fault characteristic expression. In order to verify the effectiveness of the space, frequency and time domain signal loading values of the fault characteristic factors generated by the centrifugal pump system after parallel factor analysis, the characteristic factors obtained after parallel factor analysis are used as the SPRT test sequence for identification and verification. The results indicate that the method proposed in this article improves the measurement accuracy and intelligence of mechanical fault detection.
\end{abstract}

Keywords: PARAFAC, Tensor decomposition, SPRT, Fault diagnosis, Centrifugal pump

\section{Introduction}

In recent years, equipment fault diagnosis, as a new technology that crosses various disciplines, has been developed rapidly and has produced huge economic benefits ${ }^{[1-6]}$. The centrifugal pump is an important energy conversion and liquid transmission device in the process industry and its working state directly affects the production of the entire operating equipment. The impeller of the centrifugal pump will be damaged during production and operation. A slight damage to the impeller will shorten the running time of the centrifugal pump and affect the operation of the equipment. When the impeller fails, it will cause damage to the centrifugal pump components or personal injury accidents, which will cause significant economic losses ${ }^{[7-8]}$. The normal operation and failure of the centrifugal pump will cause the equipment to vibrate. The vibration signal contains rich information of the pump body running state and is easy to be collected, which can be used to monitor and diagnose the running state of the centrifugal pump. Fault diagnosis is generally divided into three steps: first, we collect the relevant vibration signal of the diagnostic object; then, the signal is analyzed and processed to acquire the characteristics of the vibration signal; finally, pattern recognition and fault diagnosis are performed through the corresponding extracted special diagnosis ${ }^{[9-10]}$. The core content is to obtain the effective characteristics of the vibration signal. Due to the complex structure of the centrifugal pump, many excitation sources and mutual interference, the vibration signal of the centrifugal pump is a non-linear and non-stationary signal. Researchers have proposed various effective diagnostic methods to process the collected raw vibration signals of the 
centrifugal pump, extract effective information and improve the accuracy of diagnosis. Wenjian Huang et al ${ }^{[11]}$. extracted the characteristic parameters of the vibration signal through time-domain signal analysis, then the PCA was used to reduce the amount of data, and finally the main component with the largest contribution rate was used as the input signal of SPRT to evaluate the proposed algorithm. Literature ${ }^{[12]}$ proposed an improved deep convolutional neural network (CNN) to identify defects in centrifugal pumps by using sound and image recognition. A feature extraction method based on empirical mode decomposition (EMD) was developed to detect the gravity of cavitation in the centrifugal pump by Azizi et al ${ }^{[13]}$. Liu Yang et al. ${ }^{[14]}$ proposed the new method for analysis of big data based on particle swarm optimization wavelet neural network for diagnosis in the gearbox. Literature ${ }^{[15]}$ applies variational mode decomposition (VMD) with different input parameters to fault diagnosis of multistage pumps. Signal processing combined with empirical mode decomposition (EMD) and fuzzy c-means clustering is used for mornitoring piston pump defects in literature ${ }^{[16]}$. The traditional decomposition method of processing high-dimensional data will not only lose the integrity of the data but also increase the amount of calculation and introduce redundancy ${ }^{[17-20]}$. These methods of extracting time-frequency characteristics from single-channel signals, such as Fourier transform, cannot reflect the internal relationship of nonlinear changes between multi-source channel characteristic signals, nor can they eliminate information interference.

How to analyze vibration signal data effectively is a long-standing problem in fault diagnosis. At present, a popular method for component modal recognition is based on the time-varying spectrum of the vibration signal, which is the spatial/frequency/time atomic decomposition of the time-frequency spectrum of the vibration signal by PARAFAC. Parallel factor analysis proposed by Carroll, Chang ${ }^{[21]}$ and Harshman ${ }^{[22]}$ in 1970 is a three-dimensional or multidimensional signal processing algorithm that uses iterative least squares to resolve the decomposition and identification of multidimensional matrices. The general time-frequency decomposition ignores the spatial information of the vibration signal and cannot handle multi-channel data ${ }^{[23-25]}$. Data framing in the form of a three-way array indexed by channel, frequency and time allows the application of a unique decomposition called Parallel Factor Analysis (PARAFAC). The decomposition uniqueness of PARAFAC model can obtain its model parameters without ambiguity so that the PARAFAC model has important application value. As a data processing algorithm, PARAFAC model has been successfully applied in fluorescence spectroscopy, psychology, signal processing, food science and other fields. Multi-channel electroencephalogram EEG data can usually be expressed as an $\mathrm{M} \times \mathrm{N} \times \mathrm{P}$ three-way data set and the components of the three-way data array correspond to the channel (electrodes at different positions), time (data samples) and frequency components. Schmitz, S. applied PARAFAC to analyze the temporal and spatial patterns of functional connections between neurons, which was revealed in the sequence of peaks recorded in the cat's main visual cortex (area 18) ${ }^{[26]}$.This Parallel Factor Analysis was applied for decomposing EEG data into space-time -frequency components during the resting state and mental arithmetic by Miwakeichi, Fumikazu et al. ${ }^{[27]}$. Rost'akova compares non-negative Tucker decomposition with parallel factor analysis to identify and measure human brain electrical rhythms ${ }^{[28]}$. In the literature ${ }^{[29]}$, Choi, Ji Yeh proposed a new extension function PARAFAC for processing response to three-dimensional data arranged along a two-dimensional domain and one-dimensional parameters. Technically, this method combines PARAFAC with basis function expansion approximation and is applied to EEG data to prove its empirical usefulness. A parallel factor analysis study showed that the frontal lobe area with higher frequency response is the main feature of laser evoked potential in rats with chronic inflammatory pain ${ }^{[30]}$.

Parallel decomposition has attracted great attention from domestic and foreign experts and scholars, because parallel factorization can process the constructed high-dimensional data as a whole, which not only retains the overall structure information of the data, but also makes the structure more compact and easy to understand. In the literature ${ }^{[31]}$, parallel factor analysis was used as the diagnostic tool through decomposing centrifugal pump diagnostic signal into time-frequency-space modes. Considering the difficulty of extracting fault features from rolling 
bearings under strong background noise, Yang Cheng ${ }^{[32]}$ proposed a new method based on variable mode decomposition (VMD) and phase space parallel factor analysis to detect weak fault signals of rolling bearings. In order to overcome the inability to extract sparse and interpretable latent variables from batch data, literature ${ }^{[33]}$ proposed a batch three-way data array sparse model based on sparse parallel factor (SPARAFAC) decomposition. Sparse factor matrices have the potential advantage of being easy to interpret because they eliminate redundant data information and show significant variable correlation. In chemistry, medicine and food science commonly used fluorescence excitation and emission data typically contain several chemical components at different concentrations. Fluorescence spectroscopy can generate a three-way data set with the mode "sample $\times$ excitation $\times$ emission". The main purpose of the analysis of this data type is to determine which chemicals are present in each sample and their relative concentrations. Reference ${ }^{[34]}$ conducted a comparison between Parallel Factor Analysis (PARAFAC) and Support Vector Machine (SVM) to identify and distinguish the fluorescence spectrum of coconut water brands. The above results indicate that fluorescence spectroscopy combined with PARAFAC and SVM method has been proved to be a simple and rapid detection method for coconut water and other beverages. This study [35] aims to determine whether the composition or distribution of humus in lake sediments can be characterized by chemometric spectral data. This method determines the three-dimensional excitation emission matrix in the extracted humus and performs spectral analysis of the data by using Parallel Factor Analysis (PARAFAC) with classification tree and regression tree (CART).

The theory of Sequential Probability Ratio Test (SPRT) that is a branch of mathematical statistics was proposed by Abraham Wald in 1947 in order to solve the problem of sampling and acceptance of valuable military products during world War II. This method provides an approximate formula for the critical value of accepting the null hypothesis $H_{0}$ or accepting the alternative hypothesis $H_{l}$ based on the sample values obtained from each observation, and also provides the average sampling times and power function of this test method. In 1948, Abraham Wald and American statistician Wolfowitz proved that the above-mentioned sequential probability is the smallest number of sampling times required for the test in all the two types of tests whose error probability does not exceed $\alpha$ and $\beta$ respectively. The Sequential Probability Ratio Test is the most fundamental Sequential Test in Sequential analysis proposed by Abraham Wald and it has subsequently been widely developed in various fields. Almost all the hypothesis testing problems of SPRT in mechanical fault diagnosis, such as signal detection, model variable point detection, life data analysis of centrifugal pump, crack detection of gearbox, etc., can be well applied. This research ${ }^{[36]}$ were performed on actual faults in a laboratory-scale distillation plan based on artificial neural network-multilayer perceptron (ANN-MLP) and the Wald sequential probability ratio test (SPRT). In the literature ${ }^{[37]}$, Guo Peng proposed Gaussian process and SPRT wind turbine power curve modeling and monitoring. The modeling and monitoring method proposed in this paper successfully identified two wind anemometer failures and pitch system failures. Literature ${ }^{\text {[38] }}$ proposed a fault detection algorithm based on the sequential probability ratio test (SPRT) and chi-square test for redundant multi-sensor navigation systems for supersonic cruise ships (HCV).

This paper researched the theory of parallel factor analysis for tensor built from high-dimensional data and applied the diagnosis method based on multi-source feature factors for 3D time-frequency-space model reconstruction and sequential probability ratio test (SPRT). This project established the relationship between the characteristics of the nonlinear flow conditions, the source of the fault, each vibration source and the multi-source nonlinear vibration signal under the working conditions of the mechanical failure. Parallel factorization not only extracts the nonlinear time-frequency fault characteristic value of multi-source characteristic signals, but also completes the fusion optimization while maintaining data integrity. 


\section{The model and simulation}

\subsection{Parallel factorization model}

In a two-dimensional matrix, $x_{i, j}$ is generally used to represent the elements in the two-dimensional matrix (i represents any row, $\mathrm{j}$ represents any column). Similarly, in the three-dimensional matrix, we use $x_{i, j, k}$ to represent any element in the three-dimensional matrix. At present, there is no definite name naming subscript k, let's call it "page" [39]. The subscript of the three-dimensional matrix consists of three index value row, column, page composition. The left picture in Figure 1 shows the three-dimensional matrix and the right picture shows its sub-matrix. When a certain dimension in a three-dimensional matrix is fixed, it constitutes a sub-matrix of the three-dimensional matrix that is called a slice of the three-dimensional matrix along a certain dimension.
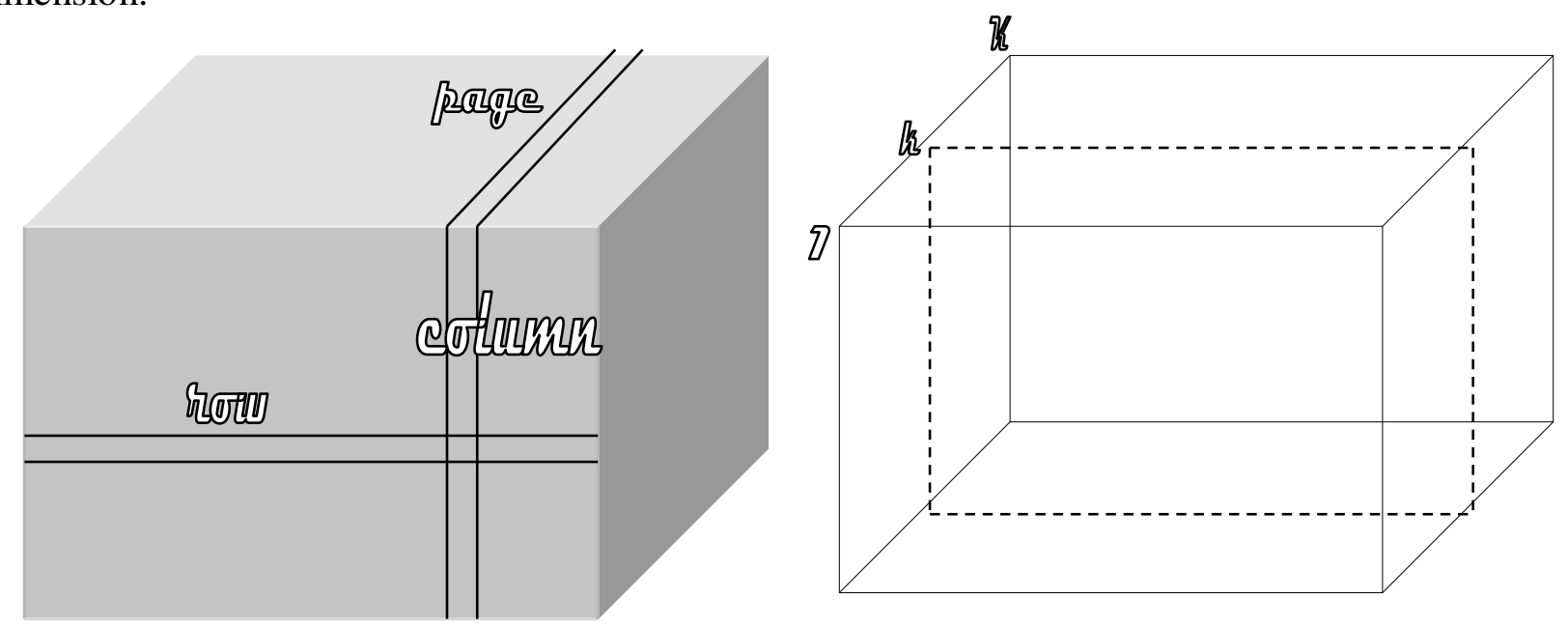

Figure 1. Three-dimensional matrix and its sub-matrix

The expansion of the three-dimensional matrix is actually to rearrange the slices of the three-dimensional matrix to constitute the new two-dimensional matrix. For example, we fixed the rows and columns of a three-dimensional matrix, rearranged its pages to formulate the new two-dimensional matrix. At this time, the number of rows is equivalent to the number of rows I of the original matrix and the number of columns changes from the original $\mathrm{J}$ to $\mathrm{J} \times \mathrm{K}$, denoted as $X^{I \times J K}$. It is expressed as shown in Equation (1).

$$
X^{I \times J K}=\left[X_{k=1}, X_{k=2} \mathrm{~L} \quad X_{k=K}\right]
$$

Of course, it can also be expanded by column, such as $X^{I K \times J}$, which is defined as formula (2).

$$
X^{I K \times J}=\left[\begin{array}{c}
X_{k=1} \\
X_{k=2} \\
\mathbf{M} \\
X_{k=K}
\end{array}\right]
$$

After expanding by columns, we acknowledge that $\mathrm{I} \times \mathrm{K}$ is displayed as the number of rows of the new matrix and parameter " $\mathrm{J}$ " is the number of columns.

The symbol $x_{i, j, k}=\sum_{f=1}^{F} a_{i, f} b_{j, f} c_{k, f} \quad$ can be used to express any element in a three-dimensional or larger than three-dimensional matrix, the variables $\mathrm{i}, \mathrm{j}$ and $\mathrm{k}$ in the formula can be any natural numbers. The elements in the i-th row, $\mathrm{j}$-th column and $\mathrm{k}$-th page of the matrix $\mathrm{X}$ can be represented by $x_{i, j, k}$. According to the definition, the low-rank decomposition of a two-dimensional matrix can be popularized to the low-rank decomposition of a three-dimensional matrix. Let the element $X \in C^{I \times J \times K}$ of the three-dimensional matrix be defined as $x_{i j k}$, the variables $\mathrm{i}, \mathrm{j}$ and $\mathrm{k}$ in the formula 
can be any natural numbers. Similarly, it can be seen that the three-dimensional matrix can be indicated as the modality of the vector outer product of the following formula (3).

$$
X=a_{1} \mathrm{o} b_{1} \mathrm{o} c_{1}+, \ldots,+a_{R} \mathrm{o} b_{R} \mathrm{o} c_{R}=\sum_{r=1}^{R} a_{r} \mathrm{o} b_{r} \mathrm{o} c_{r}
$$

It gives the process of a three dimensional matrix low rank decomposition in formula (3) and the symbol $\mathrm{R}$ of the formula is indicated as the rank of the three-dimensional matrix $\mathrm{X}$, where $c_{r} \in C^{K}$, $b_{r} \in C^{J}, a_{r} \in C^{I}, \mathrm{r}=1, \ldots, \mathrm{R}$. Harshman names the low-rank decomposition model of three-dimensional matrix given by formula (3) as the general model of parallel factor. The general forms of the parallel factorization model are interpreted in Figure 2, in which $\underline{X}$ can be displayed by the popular geometric cube.
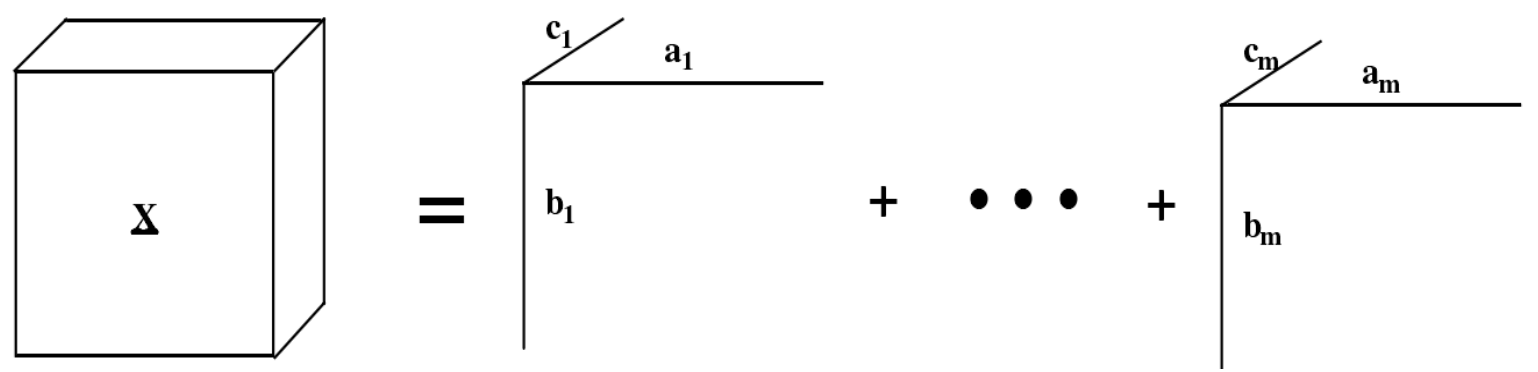

Figure 2. The general form of the parallel factorization model

These $C=\left[c_{1}, \ldots, c_{R}\right] ; B=\left[b_{1}, \ldots, b_{R}\right] ; A=\left[a_{1}, \ldots, a_{R}\right]$ are any given three two-dimensional matrix definitions. We define $\mathrm{A}, \mathrm{B}$, and $\mathrm{C}$ as the three loading matrices of the parallel factorization model of the general form. Equation (4) is shown as the scalar form of the general parallel factorization model. They are labeled as $c_{k r}=[C]_{k, r}, b_{j r}=[B]_{j, r}, a_{i r}=[A]_{i, r}$ and $\underline{x}_{i j k}=[\underline{X}]_{i, j, k}$.

$$
\underline{x}_{i j k}=\sum_{r=1}^{R} a_{i r} b_{j r} c_{k r}
$$

The general form of the parallel factorization model can be viewed as the low-rank decomposition of a two-dimensional matrix extending to a three-dimensional matrix. The formula (4) indicates that these subitems $\underline{x}_{i j k}$ of the three-dimensional matrix $\underline{X}$ can also be denoted as the sum of the products of R elements a, b and c. Compared with the matrix elements $x_{i j}$ in PCA, $\underline{x}_{i j k}$ has three independently changing dimensions called "mode A" , "mode B" and "Mode C".

\subsection{Matrix essential equalization}

There is a matrix $A \in C^{I \times J}$. If the matrix satisfies $\bar{A}=A \Pi \Delta$, it is said that $\mathrm{A}$ and $\bar{A}$ are Matrix Essential Equalization, denoted as $A \cong \bar{A}$. Among them, $\Pi$ is the column exchange matrix and $\triangle$ is the diagonal scale matrix. There is one and only one non-zero element "1" in each row and each column of the column exchange matrix $\Pi$. The function of the column exchange matrix is to rearrange the column vector of $A$ in the order of $\Pi$ without changing the value of the elements in the vector. The diagonal scale matrix $\triangle$ is a $\mathrm{J} \times \mathrm{J}$ diagonal matrix with non-zero diagonal elements. The function of $\triangle$ is to multiply each column of matrix A by a non-zero amplitude.

According to the concept of Matrix Essential Equalization, we take a two-dimensional matrix $X=A B^{T}$ as an example, where $A \in C^{M \times F}, B \in C^{N \times F}$. For any matrix $\bar{A} \in C^{M \times F}, \bar{B} \in C^{N \times F}$, if $X=A B^{T}=\bar{A} \bar{B}^{T}$ is satisfied, then we can get to formula (5).

$$
\bar{A}=A \prod_{1} \Delta_{1}, \bar{B}=B \prod_{2} \Delta_{2}
$$

$\Pi 2$ and $\Pi 2$ in the formula are column exchange matrices, which means to rearrange the columns of the A and B matrices. $\triangle_{1}$ and $\triangle_{2}$ are diagonal scale matrices, which means that each 
column of matrix A and B is multiplied by the non-zero coefficient. At this time, the matrix decomposition is said to be unique.

When the matrix factorization is unique, the matrix $\bar{A}, \bar{B}$ obtained by the matrix factorization is not completely equal to the original matrices $\mathrm{A}$ and $\mathrm{B}$, they are only the essential equality relationship of the matrix. The essential equal relationship of matrix $\mathrm{A}$ and $\mathrm{B}$ is shown in the following formula (6) (7).

$$
\begin{aligned}
& \bar{A}=A \prod_{A} \Delta_{A} \cong A \\
& \bar{B}=B \prod_{B} \Delta_{B} \cong B
\end{aligned}
$$

Due to the existence of column exchange matrices $\Pi_{\mathrm{A}}, \Pi_{\mathrm{B}}$ and diagonal scale matrices $\triangle_{\mathrm{A}}$, $\triangle_{\mathrm{B}}$, the order and magnitude of the column vectors in matrix $\bar{A}, \bar{B}$ can be different from those of $\mathrm{A}$ and $\mathrm{B}$. In matrix theory, they are used to be called column blur and scale blur, which are represented by column exchange matrix $\Pi$ and diagonal scale matrix $\triangle$, respectively. In the process of matrix decomposition, if there is no structural constraint on the matrices $\mathrm{A}$ and $\mathrm{B}$, column blur and scale blur are unavoidable. The above problem can be explained in the vector form of matrix decomposition, which is denoted $A=\left[a_{1}, \mathrm{~L}, a_{F}\right], B=\left[b_{1}, \mathrm{~L}, b_{F}\right]$, where $a_{f} \in C^{I \times 1} 、 b_{f} \in C^{J \times 1}$ $(\mathrm{f}=1, \ldots, \mathrm{F})$ is the column vector of $\mathrm{A}$ and $\mathrm{B}$ respectively. The above formula can be expressed as the following vector form.

$$
X=A B^{T}=\mathrm{a}_{1} \mathrm{~b}_{1}^{T}+\mathrm{a}_{2} \mathrm{~b}_{2}^{T}+\mathrm{L}+\mathrm{a}_{F} \mathrm{~b}_{F}^{T}
$$

In formula (8), $\mathrm{a}_{1} \mathrm{~b}_{1}^{T}, \mathrm{~L}, \mathrm{a}_{F} \mathrm{~b}_{F}^{T}$ are $\mathrm{F}$ matrices with rank 1. At this time, if the order of $\mathrm{a}_{1} \mathrm{~b}_{1}^{T}, \ldots, \mathrm{a}_{F} \mathrm{~b}_{F}^{T}$ is changed arbitrarily, the value of matrix $X$ is unchanged. Similarly, if the vector $a_{f}$ is multiplied by the non-zero coefficient $\lambda_{\mathrm{f}}$ and the corresponding $b_{\mathrm{f}}$ is multiplied by a non-zero coefficient $1 / \lambda_{\mathrm{f}}$, the value of $X$ will not change either. Assuming that the order of $a_{1} b_{1}^{T}$ and $a_{2} b_{2}^{T}$ in formula (8) is exchanged, it can be rewritten into the following form:

$$
\begin{aligned}
X & =A B^{T}=\mathrm{a}_{1} \mathrm{~b}_{1}^{T}+\mathrm{a}_{2} \mathrm{~b}_{2}^{T}+\mathrm{L}+\mathrm{a}_{F} \mathrm{~b}_{F}^{T} \\
& =\lambda_{2} a_{2} \frac{1}{\lambda_{2}} b_{2}^{T}+\lambda_{1} a_{1} \frac{1}{\lambda_{1}} b_{1}^{T}+\mathrm{L}+\lambda_{F} a_{F} \frac{1}{\lambda_{F}} b_{F}^{T} \\
& =A \prod_{A} \Delta_{A}\left(B \prod_{B} \Delta_{B}\right)^{T} \\
& =\bar{A} \bar{B}
\end{aligned}
$$

The result $X=A B^{T}=\bar{A} \bar{B}^{T}$ can be obtained, the code in the equation is specifically expressed as follows $\bar{A}=A \prod_{A} \Delta_{A}, \bar{B}=B \prod_{B} \Delta_{B}$.

It can be seen that there are always $\bar{A}$ and $\bar{B}$ to achieve matrix decomposition, but they are only essentially equal to A and B. Therefore, column ambiguity and scale ambiguity are inherent ambiguities in the matrix decomposition process. Without additional constraints, the order and magnitude of loading matrix columns cannot be determined through matrix decomposition. Therefore, the uniqueness of matrix factorization given by the definition can also be called "essential uniqueness". In the actual application process, the column blur and scale blur caused by matrix decomposition can be eliminated by some methods.

\subsection{Recognizability and uniqueness of parallel factorization}

The essential feature of the parallel factorization model is the uniqueness of the model. When there is no array blur, the matrices $\mathrm{A}, \mathrm{B}$ and $\mathrm{C}$ can be identified. The following conclusions can be obtained. When $X_{i}=B D_{i}(A) C^{T}, \mathrm{i}=1,2, \ldots, \mathrm{I}, \quad A \in C^{I \times F}, B \in C^{J \times F}, C \in C^{K \times F}$ is given, if $k_{A}+k_{B}+k_{C} \geq 2 F+2$, then these matrices $\mathrm{A}, \mathrm{B}$ and $\mathrm{C}$ are uniqueness for column exchange and plurality transformation or scale transformation.

The matrix composed of relatively independent columns taken from the absolute continuous distribution has full k-rank. If all three matrices meet this condition, the sufficient condition for 
recognizability is shown in formula (10).

$$
\min (I, F)+\min (J, F)+\min (K, F) \geq 2 F+2
$$

If the matrices $\mathrm{A}, \mathrm{B}$, and $\mathrm{C}$ have other structural constraints, better identifiable results may be obtained. PARAFAC uniqueness theorem can be used to obtain the ith submatrix of the X-axis of PARAFAC model:

$$
X_{i}^{J \times K}=B D_{i}(A) C^{T}, i=1, \ldots, I
$$

The matrix in formula (11) satisfies the following $A \in R^{I \times R}, B \in R^{J \times R}, C \in R^{K \times R}$.If the following conditions are met in Equation (12).

$$
k_{A}+k_{B}+k_{C} \geq 2(R+1)
$$

Even if there is column blur and scale blur, the matrix $\mathrm{A}, \mathrm{B}$, and $\mathrm{C}$ are unique. In mathematical language, when formula (13) is satisfied,

$$
X_{i}^{J \times K}=\hat{B} D_{i}(\hat{A}) \hat{C}^{T}, i=1, \ldots, I
$$

The relation shown in formula (14) can be obtained.

$$
\hat{A}=A \prod \Delta_{1}, \hat{B}=B \prod \Delta_{2}, \hat{C}=C \prod \Delta_{3}
$$

Equation (14) shows that $\Pi$ is a column fuzzy matrix, $\Delta_{1} \Delta_{2}$ and $\Delta_{3}$ are the scale fuzzy matrix and the equation of $\Delta_{1} \Delta_{2} \Delta_{3}=I$ needs to be satisfied.

\subsection{Trilinear Alternating Least Square for Parallel Factor Analysis}

There are many methods to achieve the decomposition of PARAFAC, and the trilinear alternate least squares (TALS) algorithm is the most widely adopted methodology for data detection of parallel factor trilinear models. The fundamental principle of the TALS is to update the matrix in each step. First of all ,TALS is updated by employing least squares (LS) to renovate the residual matrix based on the results of the previous estimate; then, it continues to update other matrices; finally, stop running until the result converges or reaches the set number of iterations after repeating the above steps. The trilinear model of three-dimensional data set $\mathrm{X}$ has the configuration shown in formula (15) below.

$$
x_{i, j, k}=\sum_{f=1}^{F} a_{i, f} b_{j, f} c_{k, f}+e_{i j k}, i=1 \mathrm{~K} I ; j=1 \mathrm{~K} J ; k=1 \mathrm{~K} K
$$

Where $\mathrm{F}$ is the number of factors, $a_{i, f}$ is the $\mathrm{i}$-th element in vector $a_{f}, b_{j, f}$ is the $\mathrm{j}$-th element in vector $b_{f}$, and $c_{k, f}$ is the $\mathrm{k}$-th element in vector $c_{f}$. The data set $\mathrm{X}$ of third-order tensor $\mathrm{I} \times \mathrm{J} \times \mathrm{K}$ is indicated as" $x_{i, j, k}$ ". The" $e_{i j k}$ " represents the error set $\mathrm{E}$ of the third-order tensor $\mathrm{I} \times \mathrm{J} \times \mathrm{K}$. Equation $\mathrm{A}=\left[a_{1}, a_{2}, \mathrm{~L}, a_{I}\right]$ is defined as the $I \times F$ matrix; The $\mathrm{B}=\left[b_{1}, b_{2}, \mathrm{~L}, b_{J}\right]$ and $\mathrm{C}=\left[c_{1}, c_{2}, \mathrm{~L}, c_{K}\right]$ are defined as the $J \times F$ matrix and the $K \times F$ matrix.

(1) First, matrix A is calculated by formula (16).

$$
\left[\begin{array}{l}
X_{\mathrm{K} 1} \\
X_{\mathrm{K} 2} \\
\mathrm{M} \\
X_{\mathrm{K} K}
\end{array}\right]=\left[\begin{array}{l}
B \operatorname{diag} C(1,:) \\
B \operatorname{diag} C(2,:) \\
\mathrm{M} \\
B \operatorname{diag} C(K,:)
\end{array}\right] A^{T}+E_{K}
$$

Formula (16) satisfies $X_{\mathrm{K} k}=B \operatorname{diag}(C(k,:)) A^{T}+E_{\mathrm{K} k}$, The error is expressed in terms of $E_{K}$. The least squares(LS) estimate of $A^{T}$ is calculated by Equation (17). 


$$
A^{T}=\left[\begin{array}{l}
\operatorname{Bdiag} C(1,:) \\
\operatorname{Bdiag} C(2,:) \\
\mathrm{M} \\
\operatorname{Bdiag} C(K,:)
\end{array}\right]^{\div}\left[\begin{array}{l}
X_{\mathrm{K} 1} \\
X_{\mathrm{K} 2} \\
\mathrm{M} \\
X_{\mathrm{K} K}
\end{array}\right]
$$

The generalized inverse in formula (17) is represented by [ ]

(2) Secondly, matrix B is calculated by formula (18).

$$
\left[\begin{array}{l}
Y_{\mathrm{K} 1} \\
Y_{\mathrm{K} 2} \\
\mathrm{M} \\
Y_{\mathrm{K} I}
\end{array}\right]=\left[\begin{array}{l}
\operatorname{Cdiag} A(1,:) \\
\operatorname{Cdiag} A(2,:) \\
\mathrm{M} \\
\operatorname{Cdiag} A(I,:)
\end{array}\right] B^{T}+E_{I}
$$

Formula (18) satisfies the following $Y_{\mathrm{K} i}=\operatorname{Cdiag}(A(i,:)) B^{T}+E_{\mathrm{K} i}$, The error is expressed in terms of $E_{I}$. The least squares(LS) estimate of $B^{T}$ is calculated by Equation (19).

$$
B^{T}=\left[\begin{array}{l}
\operatorname{Cdiag} A(1,:) \\
\operatorname{Cdiag} A(2,:) \\
\mathrm{M} \\
\operatorname{Cdiag} A(I,:)
\end{array}\right]^{\div}\left[\begin{array}{l}
Y_{\mathrm{K} 1} \\
Y_{\mathrm{K} 2} \\
\mathrm{M} \\
Y_{\mathrm{K} I}
\end{array}\right]
$$

(3) Thirdly, matrix $\mathrm{C}$ is calculated by formula (20).

$$
\left[\begin{array}{l}
Z_{\mathrm{K} 1} \\
Z_{\mathrm{K} 2} \\
\mathrm{M} \\
Z_{\mathrm{K} J}
\end{array}\right]=\left[\begin{array}{l}
\operatorname{Adiag} B(1,:) \\
\operatorname{Adiag} B(2,:) \\
\mathrm{M} \\
\operatorname{Adiag} B(J,:)
\end{array}\right] C^{T}+E_{J}
$$

Where $Z_{\mathrm{K} j}=\operatorname{Adiag}(B(j,:)) C^{T}+E_{\mathrm{K} j}, \mathrm{j}=1,2, \mathrm{~L}, J$, . The error is expressed in terms of $E_{J}$. The least squares estimate of $C^{T}$ is calculated by Equation (21).

$$
C^{T}=\left[\begin{array}{c}
\operatorname{Adiag} B(1,:) \\
\operatorname{Adiag} B(2,:) \\
\mathrm{M} \\
\operatorname{Adiag} B(J,:)
\end{array}\right]^{\div}\left[\begin{array}{c}
Z_{\mathrm{K} 1} \\
Z_{\mathrm{K} 2} \\
\mathrm{M} \\
Z_{\mathrm{K} J}
\end{array}\right]
$$

(4) Finally, stop running until the result converges or reaches the set number of iterations after repeating the above steps(1)-(3).

This article mainly studies the vibration signal of the equipment, which obtains the data matrix through continuous wavelet transform. Figure 3 shows the fundamental structure of the decomposition model of Parallel Factor Analysis for fault diagnosis. The $N_{t}, N_{d}$ and $N_{f}$ of the data matrix $S_{\left(N_{d} \times N_{f} \times N_{t}\right)}$ are the number of data points ,the number of channels and the frequency step size, respectively.

$$
\hat{S}_{d f t}=\sum_{k=1}^{N_{k}} a_{d k} b_{f k} c_{i k}+e_{d f t}
$$

The key issue of this parallel factorization model is to obtain the matrices A, B and C. The $a_{d k}$, $b_{f k}$ and $c_{t k}$ are their elements, in which component $\mathrm{k}$ represents an atom. These spatial signals, spectral signals and temporal signals for each atom are indicated as the $a_{k}=\left\{a_{d k}\right\}, b_{k}=\left\{b_{f k}\right\}$ and 
$c_{k}=\left\{c_{t k}\right\}$. The " $e_{i j k} "$ is the error, which forms error set $\mathrm{E}$ of the third order tensor $\mathrm{I} \times \mathrm{J} \times \mathrm{K}$. The uniqueness of the solution of parallel factor decomposition for fault diagnosis is guaranteed through $\operatorname{rank}(A)+\operatorname{rank}(B)+\operatorname{rank}(C) \geq 2 N_{k}+2$. The decomposition of formula (22) is achieved by solving $\min _{a_{d k} b_{j k} c_{i k}}\left\|\hat{S}_{d f t}-\sum_{k=1}^{N_{k}} a_{d k} b_{f k} c_{t k}\right\|$. The main advantage of this method is that the spectrum decomposition of time-varying vibration signal is unique and the best model can be obtained under the principle of minimum square deviation.

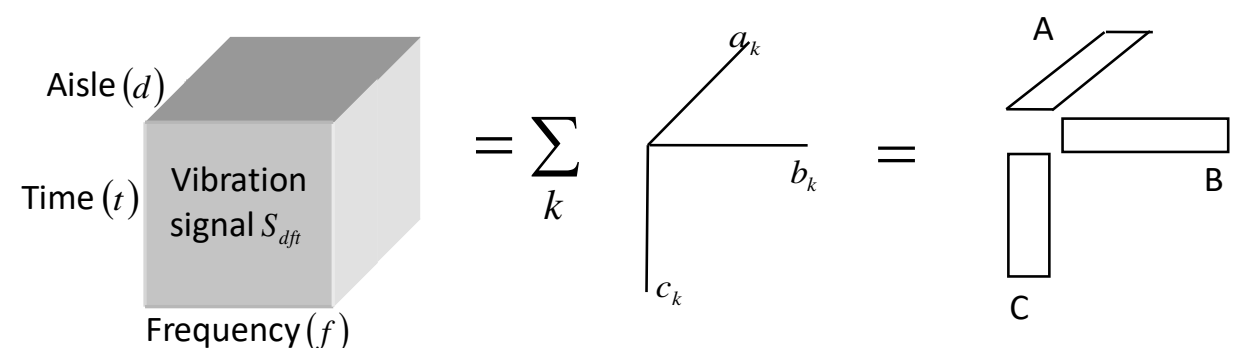

Figure 3. The model of multi-scale parallel factor decomposition for fault diagnosis

The basic steps for implementing the multi-scale parallel factorization model of fault diagnosis are as follows .

(1) After the vibration signal is collected, the third order tensor is constructed by continuous wavelet transform.

(2) The number of factor $F$ is determined by the principle of consistency in Matlab.

(3) Initialization for load matrix B and C.

(4) After initializing and running the matrices B and C, the matrix A is estimated by the least square regression algorithm. $A=X Z^{\prime}\left(Z Z^{\prime}\right)^{-1}, Z=(b \otimes c)$.

(5) Similarly, the matrices B and C are estimated.

(6) Continue from step (3) until the result converges or reaches the set number of iterations.

(7) Corresponding results obtained.

\subsection{Numerical simulation based on parallel factor analysis}

Simulation experiments can investigate the characteristic of the results of input signals with different parameters after parallel factor analysis for fault diagnosis. Therefore, the simulation signals are used to simulate the running state of the centrifugal pump to test the method proposed in this article. The simulation signal is shown in the following formula (23).

$$
\left.\mathrm{y}(\mathrm{t})=0.01 * \cos \left(2 * \mathrm{pi}^{*} 400 * \mathrm{t}-5\right) * \exp \left(-0.5 *((\mathrm{t}-0.03) / 0.003)^{\wedge} 2\right)\right)
$$

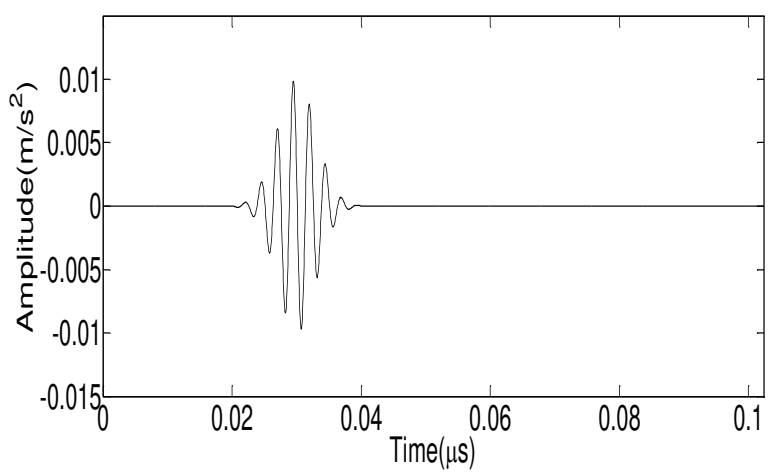

Figure4. Time-domain diagram of the original simulated signal

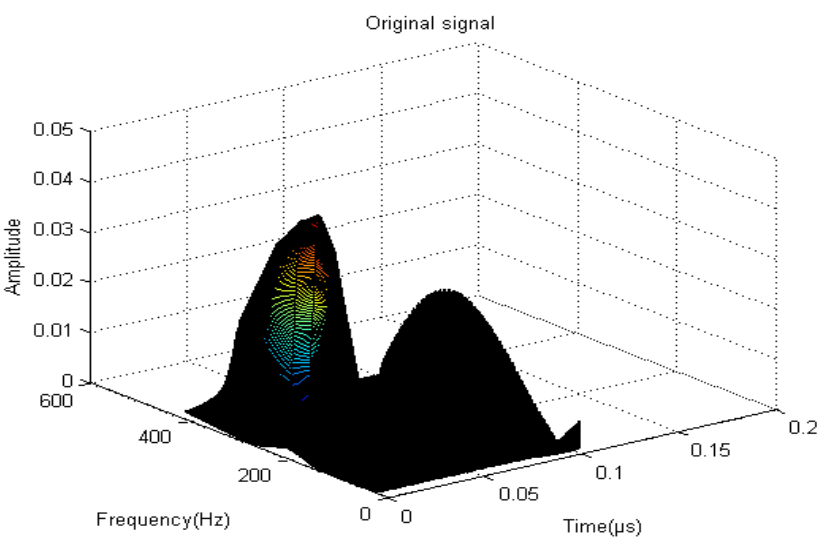

Figure 5. Time-frequency diagram of original simulation signal

Figure 4 shows the time-domain diagram of the simulated signal. An impact signal appears in the graph at $0.03 \mu \mathrm{s}$, which simulates the signal generated when the system fails. It is shown in 
Figure 5 that the time-frequency diagram of the simulation signal is obtained by the continuous wavelet transform. It can be seen from the time-frequency diagram of the simulation signal that the dominant frequency of the signal is $400 \mathrm{HZ}$ and the impact signal in the simulation signal appears in the frequency range of $180-400 \mathrm{HZ}$. For the time-frequency diagram of the simulated signal in Figure 5, it can also be seen that the impact signal appears at $0.03 \mu \mathrm{s}$.
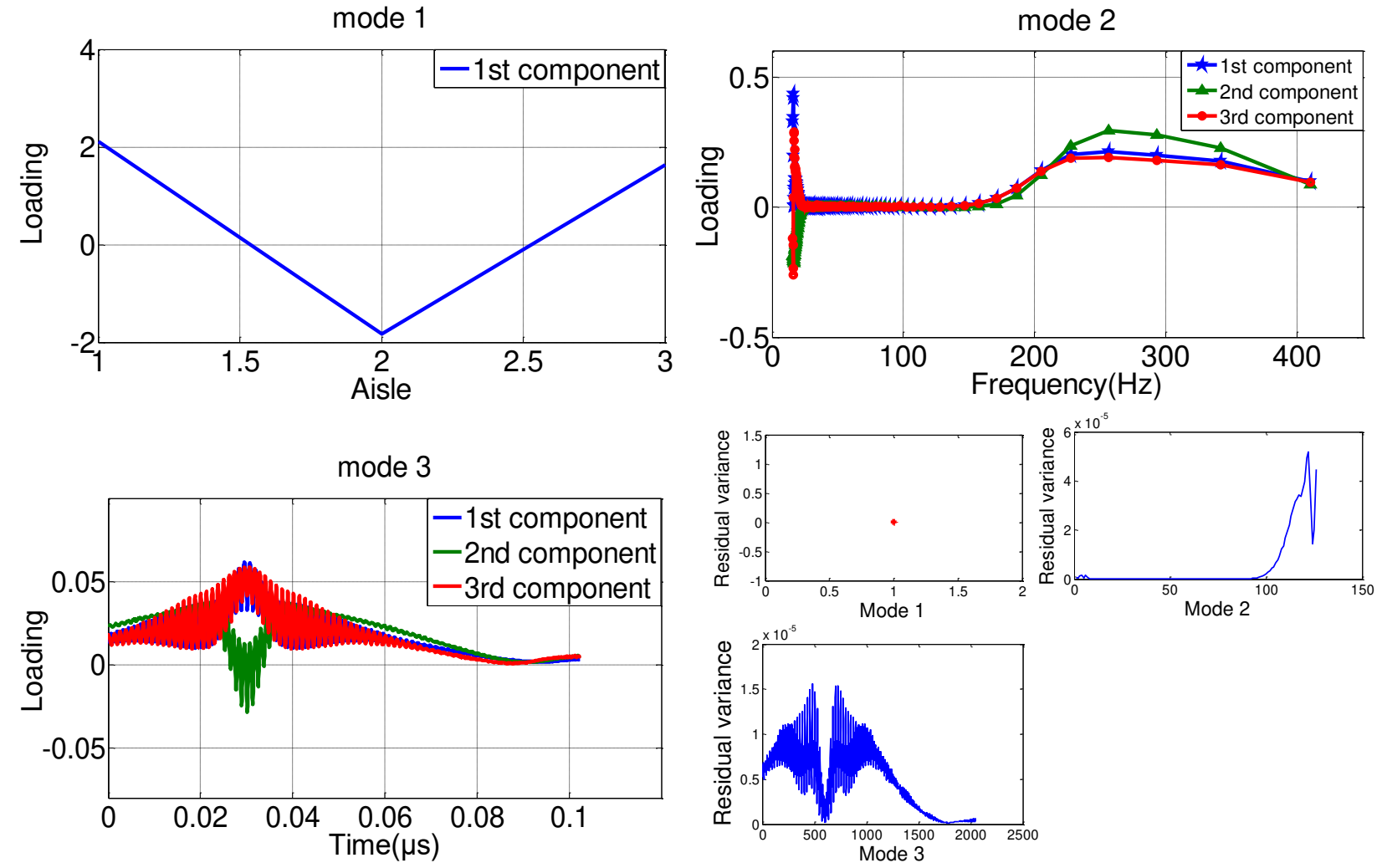

Figure 6. Parallel factor analysis of the original simulation signal

The time-frequency result of the above simulation signal is constructed into a third-order tensor after continuous wavelet transformation, and then the third-order tensor is analyzed by parallel factors to obtain the result shown in Figure 6. After parallel factor analysis, we can get the loading value and residual error corresponding to frequency, time and channel. Comparing the loading value of frequency and time after parallel factor analysis with the time-frequency diagram, we can find their corresponding relationship. The frequency curve in the graph fluctuates in the range of $180-400 \mathrm{HZ}$, which is a contrast relationship between the fluctuations of the simultaneous frequency graph. The time curve fluctuates at $0.03 \mu \mathrm{s}$ and has the maximum value of the third component and the minimum value of the second component. It can be seen from the simulation signal corresponding to the ground that the simulation signal also has an impact signal at $0.03 \mu \mathrm{s}$. This indicates that the parallel factor analysis proposed in this article for high-dimensional data of multi-source feature factors can well detect the characteristics of the shock signal generated by the simulated fault.
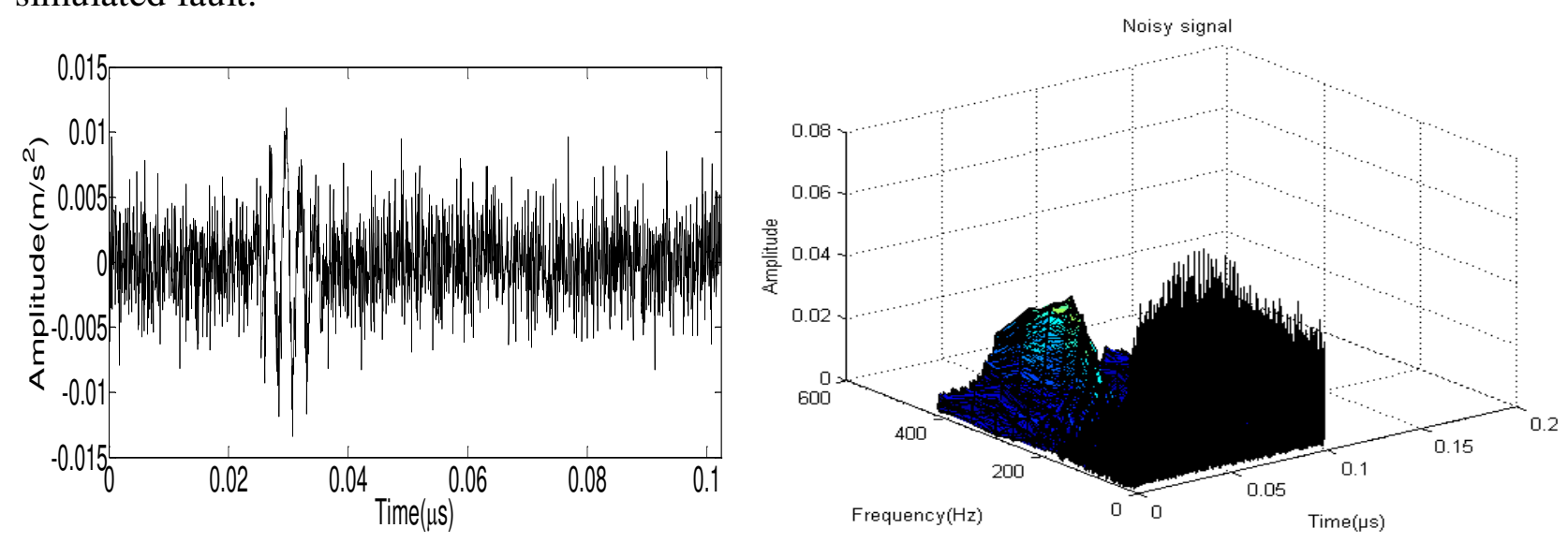
Figure 7. Time-domain diagram of noise-added simulation signal
Figure 8. Time-frequency diagram of noise-added simulation signal

The vibration signals collected in engineering inspection are generally mixed with various noise signals. In order to check on the effectiveness in complex conditions, we add the noise signal to the original simulation signal and perform parallel factor analysis on it. Figure 7 and Figure 8 respectively show the time-domain diagram of the original simulation signal after adding noise to the signal and the time-frequency diagram obtained through continuous wavelet transform. After adding the noise signal to the original simulation signal, it can be seen that the waveform of the noisy simulation signal is similar to the original simulation signal in Figure 4 and the impact signal is almost covered by the noise signal. The waveform of the noisy simulation signal in Figure 8 is steeper and more rapid and there is a larger blurred signal at 10-20HZ.

mode 1

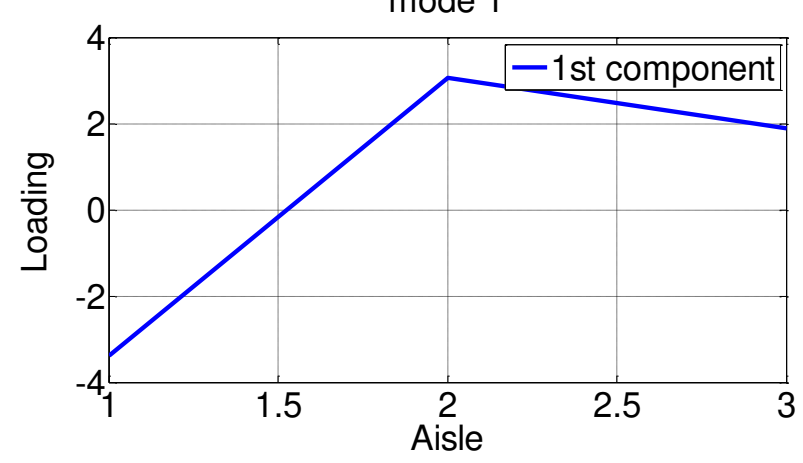

mode 3

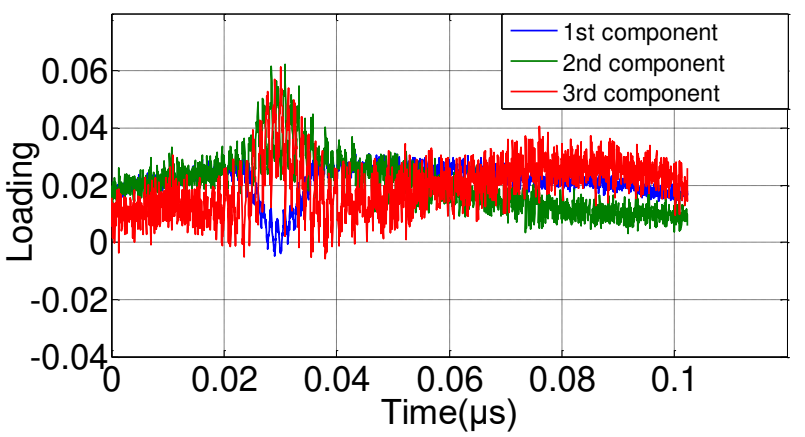

mode 2
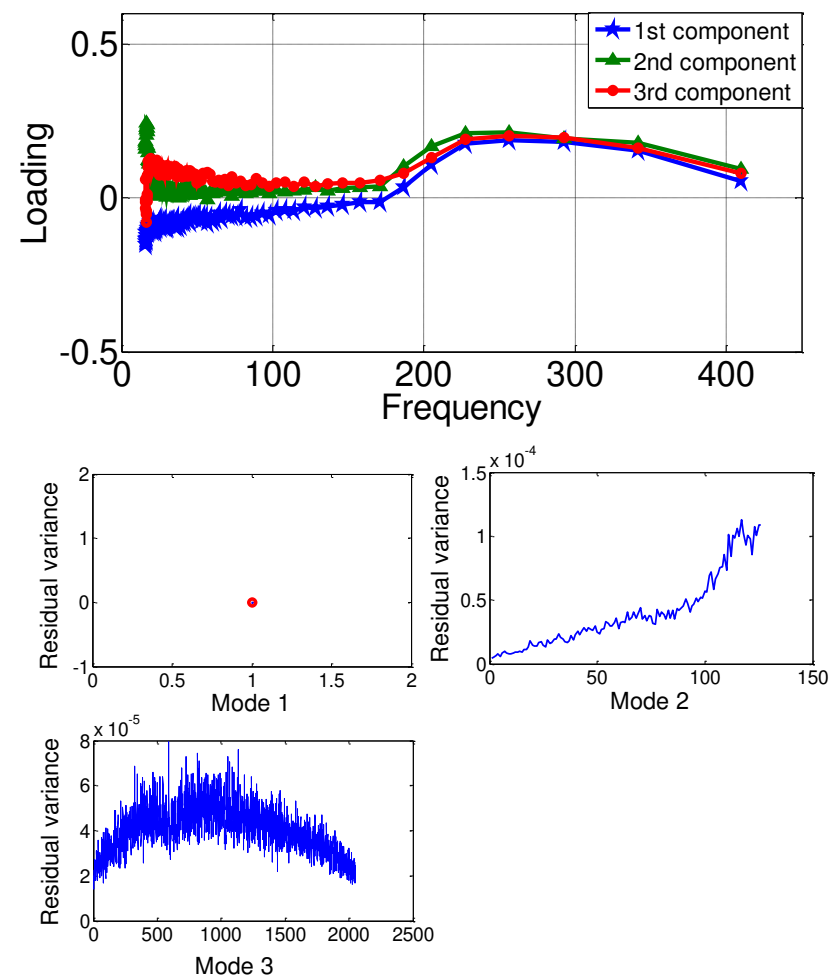

Figure 9. Parallel factor analysis of noise added simulation signal

The simulation signal with noise is transformed into a third-order tensor after continuous wavelet transformation. The result of the parallel factor analysis of the third-order tensor is shown in Figure 9. After parallel factor analysis, we can get the loading value and residual parameters corresponding to frequency, time and channel. Comparing the loading value of frequency and time after parallel factor analysis with the time-frequency diagram, we can find the correspondence between them relationship. The frequency curve in the graph fluctuates in the range of 180-400HZ, which is a contrast relationship between the fluctuations of the simultaneous frequency graph . The time curve fluctuates at $0.03 \mu \mathrm{s}$ and has a maximum value. We get the normal probability plot and the residual variance corresponding to the data in Mode1, Mode2 and Mode3. This shows that the parallel factor analysis proposed can well detect the characteristics of the impact signal in this paper even when the collected signal contains noise.

\section{Proposed method}

Likelihood function is a function of statistical model parameters, which plays a great role in statistical inference. The general method of using likelihood ratio test statistics was proposed by Neyman-Pearson in $1982^{[40]}$. Its basic idea is similar to the maximum likelihood method of parameter estimation theory, which is called likelihood ratio test. For hypothesis $H_{0}: \theta=\theta_{0}$, 
alternative hypothesis $H_{1}: \theta=\theta_{1}$, $\mathrm{x}$ is a set of random variables. When $H_{0}$ is true, the probability density function of the random variable $\mathrm{x}$ is expressed as $f\left(x, \theta_{0}\right)$. When $H_{1}$ is true, the probability density function of the random variable $\mathrm{x}$ is expressed as $f\left(x, \theta_{1}\right)$. The likelihood function of the sample is the following formula(24).

$$
L(\theta)=\prod_{i=1}^{n} f\left(x_{i}, \theta\right)
$$

Therefore, the likelihood ratio test is performed to obtain the statistic $\mathrm{L}$ in the following formula (25).

$$
L=\frac{L\left(\theta_{1}\right)}{L\left(\theta_{0}\right)}=\frac{\prod_{i=1}^{n} f\left(x_{i}, \theta_{1}\right)}{\prod_{i=1}^{n} f\left(x_{i}, \theta_{0}\right)}
$$

If the likelihood ratio $\mathrm{L}$ is larger, the parameter $\theta$ is more likely to be $\theta_{1}$, it shows that the result may tend to negate $H_{0}$. On the contrary, if the ratio is smaller, the parameter $\theta$ is more likely to be $\theta_{0}$, which indicates that the result may be inclined to accept $H_{0}$.For a certain limit k, $L$ is defined as shown in the following formula (26).

$$
\varphi(x)= \begin{cases}1 & l>k \\ 0 & l \leq k\end{cases}
$$

Test $\varphi(x)$ is called the likelihood ratio test of the above test problem.

Neyman-Pearson proposes a principle to determine the optimal test method: parameter $\alpha$ satisfies formula (27).

$$
\beta(\theta) \leq \alpha \quad \forall \theta \in \Theta_{0}
$$

In formula (27), $\beta(\theta)$ is the power function of the test, $\Theta_{0}$ is the parameter space of the null hypothesis $H_{0}$, and $\theta$ is the test parameter. Look for a test that satisfies the above formula so that $\beta(\theta)$ is as large as possible when $\theta \in \Theta_{0}$. To ensure that the probability of making two types of errors is very small, the sample size must be increased. For field testing, the smaller the sample size, the better when ensuring the reliability of the conclusion. The sequential method proposed by A. Wald solves the problem of optimal selection of sample size and play an important milestone in the history of statistical development.

The probability function $f(x, \theta)$ represents the distribution of the random variable $\mathrm{x}$, $H_{0}\left(\theta=\theta_{0}\right)$ and $H_{1}\left(\theta=\theta_{1}\right)$ are the null hypothesis and alternative hypothesis of the random variable $\mathrm{x}$, respectively. When accepting $H_{1}$, the probability of the sample $x_{1}, \ldots, x_{m}$ for any positive integer $m$ is given by $P_{1 m}=f\left(x_{1}, \theta_{1}\right), \ldots, f\left(x_{m}, \theta_{1}\right)$, and the probability is given by $P_{0 m}=f\left(x_{1}, \theta_{0}\right), \ldots, f\left(x_{m}, \theta_{0}\right)$ when accepting $H_{0}$. The definition of the sequential probability ratio test is as follows: select two normal numbers $\mathrm{A}$ and $\mathrm{B}(\mathrm{B}<\mathrm{A})$ and calculate the probability ratio $P_{1 m} / P_{0 m}$ at each stage of the test.

(a)If $p_{1 m} / p_{0 m} \geq A$, the sequential probability ratio test ends, $H_{1}$ is accepted and $H_{0}$ is discarded.

(b)If $p_{1 m} / p_{0 m} \leq B$, the sequential probability ratio test ends, $H_{0}$ is accepted and $H_{1}$ is discarded.

(c)If $B<p_{1 m} / p_{0 m}<A$, we continue to observe the sequential probability ratio test until the requirement is met.

When SPRT is applied to target recognition, it is first assumed that one of the M alternative hypotheses is the initial hypothesis. The signal propagation waveform is denoted as $s(t)$. When a signal is transmitted, one of the possible waveforms is received and recorded as follows: 


$$
y(t)=s(t) * h_{i}(t)+n(t) \quad i \in\{1,2, \mathrm{~K}, M\}
$$

Where $n(t)$ is additive white Gaussian noise; the impulse response of the target hypothetical channel is expressed as $h_{i}(t)$ and "*" is the convolution factor.

The signal channel receiving data is defined in formula (29), where $Q_{i}$ represents the target convolution matrix defined in the literature.

$$
y=Q_{i} s+n
$$

The $\mathrm{M}$ target hypotheses are denoted as $H_{1}, H_{2}, \ldots, H_{M}$ respectively. The parameter $\alpha_{i, j}$ is the probability $(i \neq j)$ when the true hypothesis $H_{i}$ is wrongly selected as $H_{j}$. After obtaining $k^{\text {th }}$ observations, suppose the likelihood ratio of $\mathrm{i}$ and $\mathrm{j}$ can be defined as shown in formula (30)

$$
\Lambda_{i, j}^{k}=\frac{p_{i 1}\left(y_{1}\right) p_{i 2}\left(y_{2}\right) \Lambda p_{i k}\left(y_{k}\right)}{p_{j 1}\left(y_{1}\right) p_{j 2}\left(y_{2}\right) \Lambda p_{j 2}\left(y_{k}\right)} \frac{P_{i}}{P_{j}}
$$

Where $p_{i k}\left(y_{k}\right)$ is the probability density function (PDF) with $k^{\text {th }}$ data under the $i^{\text {th }}$ hypothesis and $y_{k}$ is the $k^{\text {th }}$ observation data. When the likelihood ratio satisfies formula (31), accept the assumption $H_{m}$.

$$
\Lambda_{i, j}^{k}>\frac{1-\theta_{i, j}}{\theta_{i, j}} \quad j \neq i
$$

When the likelihood ratio satisfies the formula (31), stop the loop. If the likelihood ratio does not meet the stopping condition, continue to the next iteration. In fact, the probability density function of the observed data is constant and satisfies $p_{i 1}(y)=p_{i 2}(y)=\ldots=p_{i k}(y)$. The intensity waveform is updated with the number of iterations, so the probability density function of the observation data under the condition of additive white Gaussian noise can be defined as formula (32).

$$
p_{i k}\left(y_{k}\right)=\frac{1}{\left(\sqrt{2 \pi \sigma_{n}^{2}}\right)^{L_{y}}} \times \exp \left[-\frac{1}{2 \sigma_{n}^{2}}\left(y_{k}-Q_{i} s_{k}\right)^{T}\left(y_{k}-Q_{i} s_{k}\right)\right]
$$

\section{Experiments}

\subsection{Slurry pump fault test system and experimental design}

The experimental system to be established in this project is required to operate the slurry pump under controlled conditions of speed, flow rate, slurry density and inlet pressure, and to use and replace the impeller of the slurry pump of different grades and wear. Common failure parts of centrifugal pumps include rotor impeller, rolling bearing, seal, coupling, etc., of which impeller and rolling bearing failure account for a large proportion. The schematic diagram of the slurry pump fault diagnosis test system is shown in Figure 10. The figure shows the three-dimensional schematic diagram of the test circuit and identifies the key components. It mainly includes motors, slurry pumps, density meters, glycol cooling tanks, pressure gauges, flow meters, conveyor belts, sand tanks, data acquisition systems, pipelines, pressure control tanks, sampling ports and control instruments. First, the normal impeller is used in the centrifugal slurry pump to run the slurry pump fault test system for collecting and testing the signal data of the slurry pump vibration, flow, slurry density, motor speed and pump inlet and outlet pressure. Then impeller perforation, impeller edge damage and blade damage and its impellers with different degrees of damage were selected to replace the original centrifugal pump impeller. After running the slurry pump fault diagnosis and test system, the data of the vibration, speed and pump speed of the mud pump experiment system were collected. 


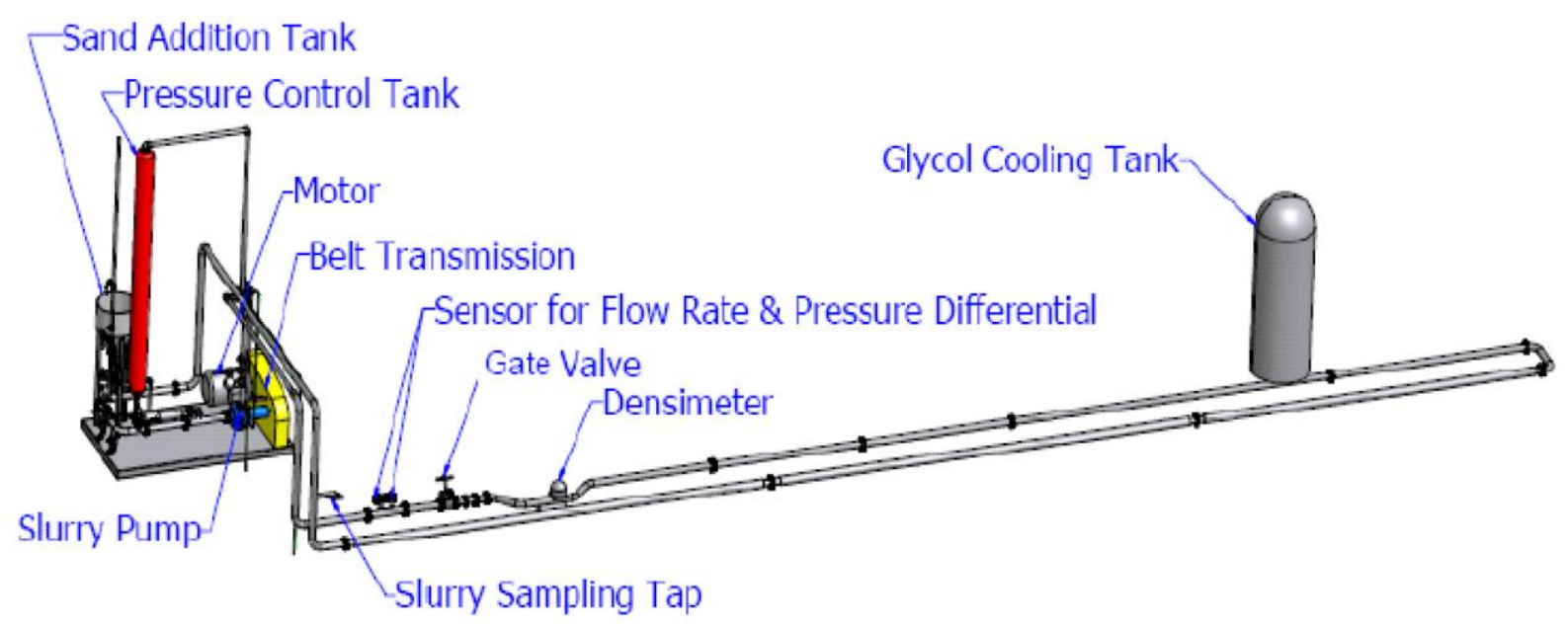

Figure 10. Schematic diagram of slurry pump fault diagnosis test system

Figure 11 shows the process flow chart of the slurry pump fault diagnosis test system. The arrow direction in the figure is the flow direction of the mud when the mud pump fault diagnosis experiment system is running. It is the basis for establishing and running the centrifugal pump fault diagnosis experiment system in this article. The serial number and related schematic diagram in Figure 11 indicate the following meanings: 1- centrifugal pump, 2-motor, 3-inverter, 4-power meter sensor, 5-accelerometer, 6-pressure sensor, 7-flow meter, 8-hole plate, 9-heat exchanger, 10-cooler, 11-temperature sensor, 12-sand, 13-suction pressure control tank, 14-suction pressure sensor. The fault diagnosis test system for slurry pump contains a Weir/Warman 3/2 CAH slurry pump (40 HP) with impeller C2147(8.4"). The Process flow chart of fault diagnosis test system for slurry pump covers the key issues mentioned in this article, but does not cover all aspects of the design of the experimental system. The key issues include that the medium of the cooler in the pipeline is ethylene glycol, the process water is municipal water, and the heat exchanger medium is steam. Microphone means for sound collector.

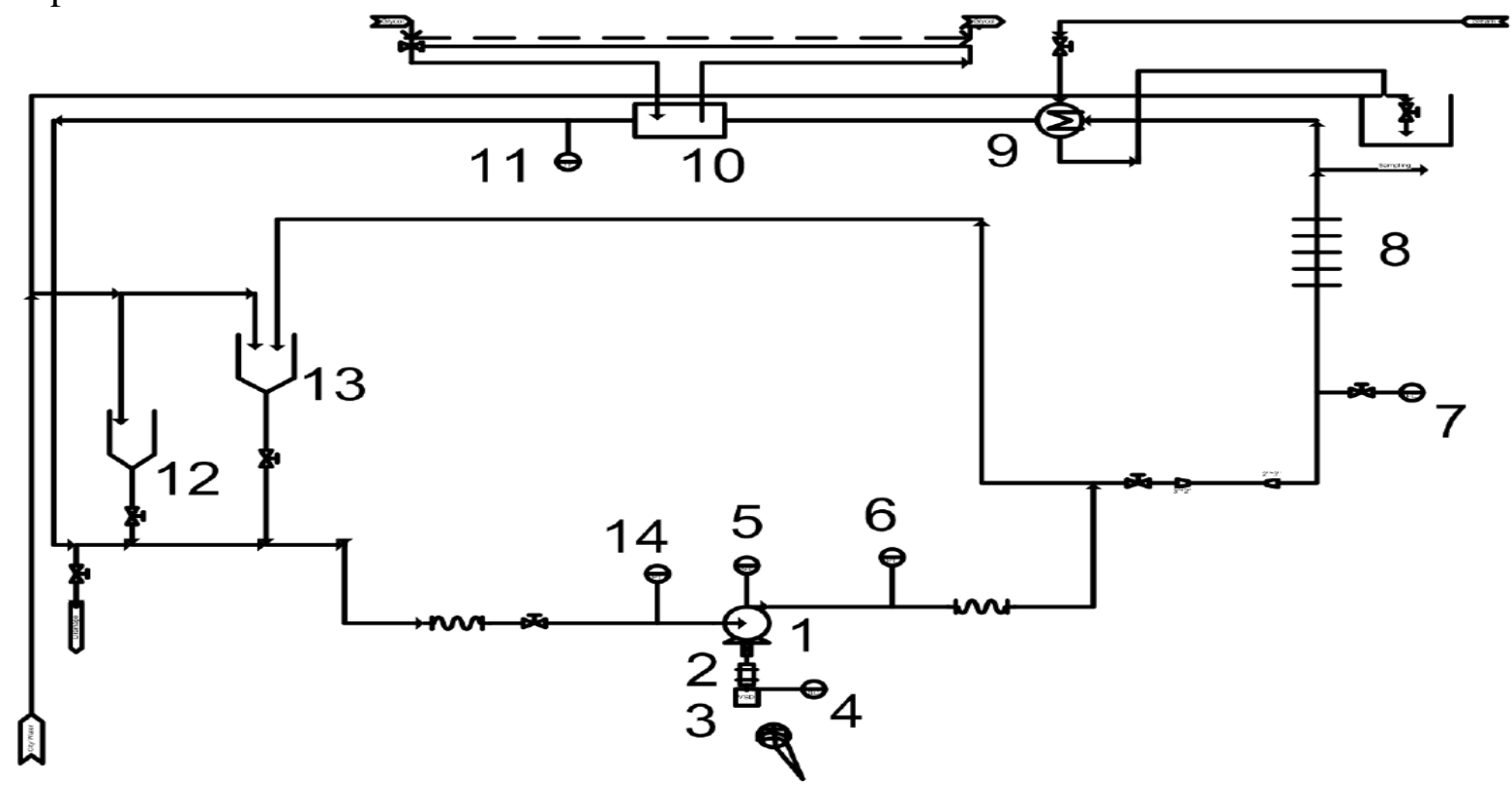

Figure 11. Process flow chart of fault diagnosis test system for slurry pump

In order for the experiment to run successfully, the designer first needs to design the system after engineering calculation and determine the components. The main equipment required for the experiment includes the centrifugal pump, data acquisition system for vibration data acquisition, sensors and a laptop computer. Auxiliary equipment including storage tanks, valves, instruments and drive motors are used to control various functions. The data collected by vibration 
accelerometers is used to analyze the centrifugal pump system in this experiment. The detailed explanation of the vibration sensor for the system signal acquisition is shown below. In the experiment, three three-axis vibration accelerometers are used. Two of the PCB three-axis ICP (Integrated Circuit Piezoelectric) accelerometers have the sensitivity of $100 \mathrm{mV} / \mathrm{g}$ and the frequency range of $2-5 \mathrm{kHz}$. Another PCB three-axis ICP accelerometer has the range of $0.5-3 \mathrm{kHz}$ and the sensitivity of $1000 \mathrm{mV} / \mathrm{g}$.

\subsection{Slurry pump experimental equipment and signal acquisition system}

To research the validity of the multi-scale parallel factor analysis and sequential probability ratio test proposed in this paper in the actual industrial production, the centrifugal pump fault diagnosis experimental system was designed. The general figure 12(a) shows the centrifugal pump fault diagnosis experimental system. The data acquisition system is shown in Figure 12(b) based on a combination of PC measurement hardware and software, which can input electrical signals from sensors and other instruments into a computer for processing. NI LabView 7.0 was chosen as the measurement standard application software because it is easy to build a graphical measurement interface with the help of a large number of tools and objects. The selected hardware is provided by NI DAQ and is highly compatible with our software applications. In order to collect the vibration signals of the centrifugal pump in three directions for each state, it is necessary to install a short-range but high-sensitivity sensor at the key position. Figure 12 (d) is a schematic diagram of the position of the accelerometer. The standard accelerometer and the high-sensitivity accelerometer are installed on the pump casing near the pump suction port, where they will be close to the parts that are prone to failure. Another standard accelerometer is mounted on the shaft bearing because this location is sensitive to vibrations transmitted from the stuffing box. Real-time signals such as flow rate, pressure, speed, vibration, etc. can be collected synchronously by the experimental system. By commanding the pressure and the flow of the equipment's loop, we simulate the non-linear operating state of the industrial process of the mechanical system to established the non-linear multi-fault mode, synchronously collect multi-channel signals and obtain multi-source signals. The internal interaction mechanism between fluid excitation and vibration response under nonlinear operation mechanism can be analyzed.

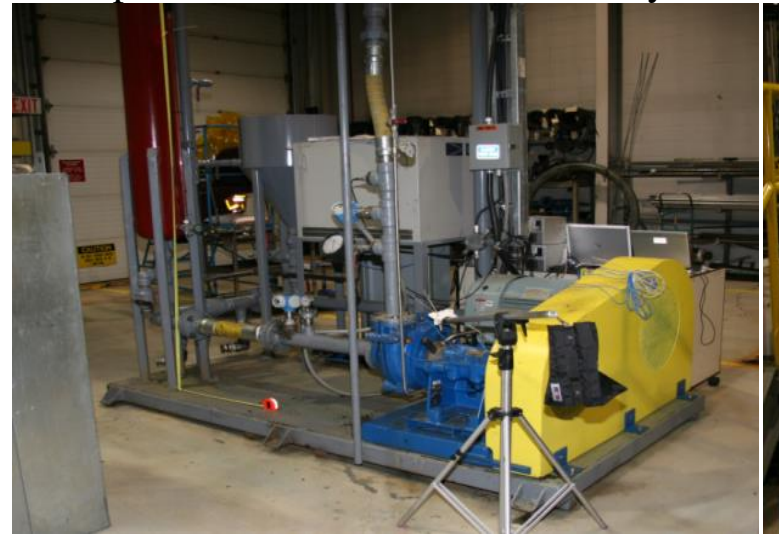

a. Centrifugal pump experimental equipment

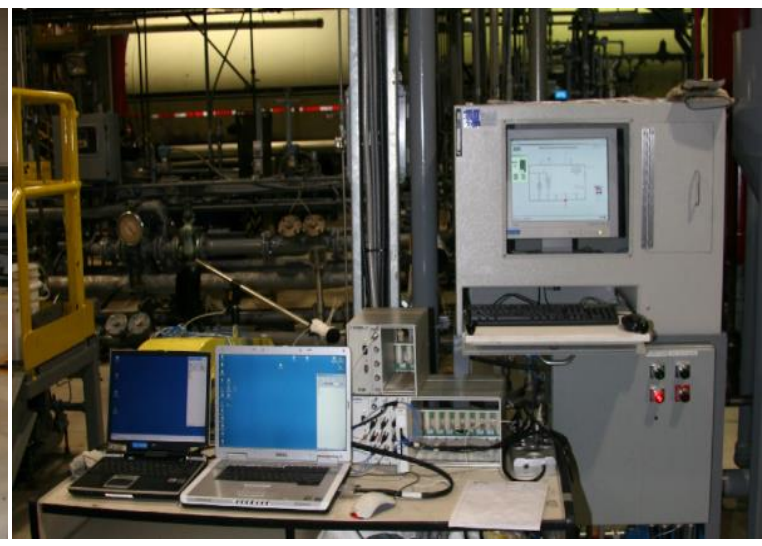

b. Signal acquisition system 


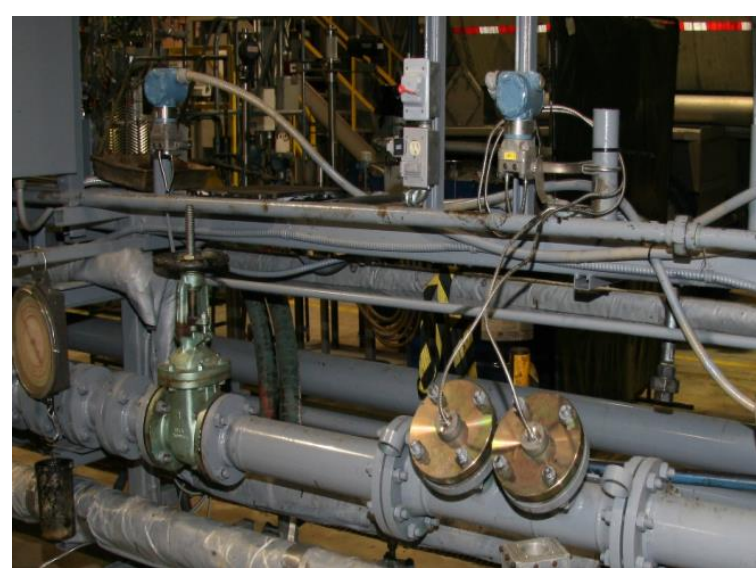

c. Sensor for flow rate

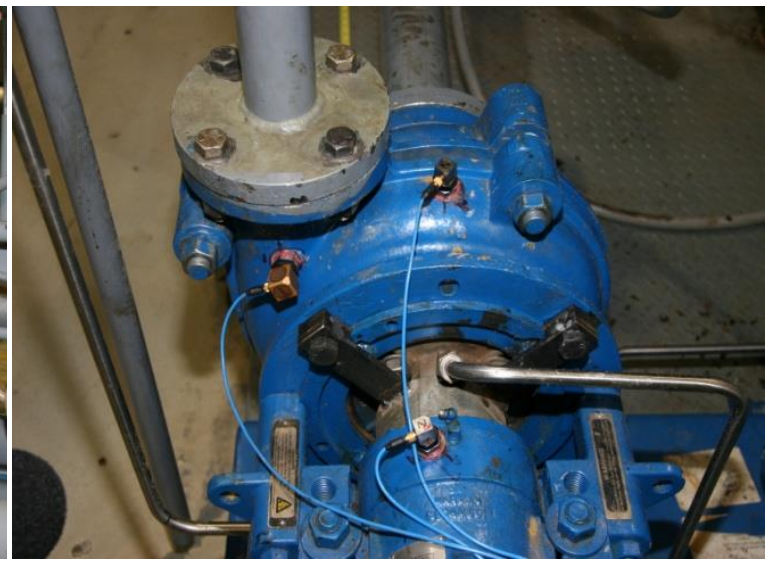

d. Installation diagram of acceleration sensor

Figure 12. Experiment system of slurry pump

The liquid transported in this experiment is set as mud to better collect vibration signals. In this experiment, normal impeller and three types of faulty impellers, including impeller perforation , impeller edge damage and blade damage, were set to simulate failures in industrial production. Among them, these three failure modes have clear differences and the typical failures of centrifugal pump impellers can be represented well. The impeller in the normal state is denoted as $S 1$, and the three types of impellers with impeller perforation, impeller edge damage and blade damage are denoted as S2, S3 and S4. In order to avoid aliasing, the sampling frequency in this experiment is $9009 \mathrm{~Hz}$ according to the Nyquist sampling theorem and the data acquisition time is 20s for each group.

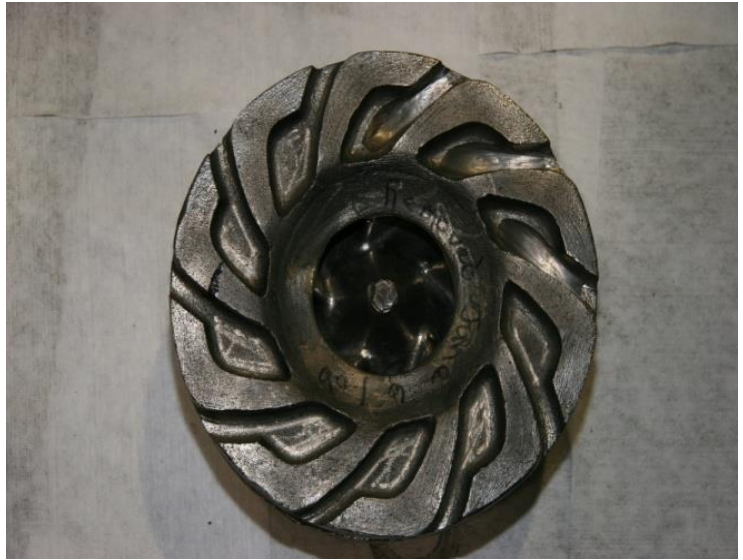

(a) S1 normal impeller

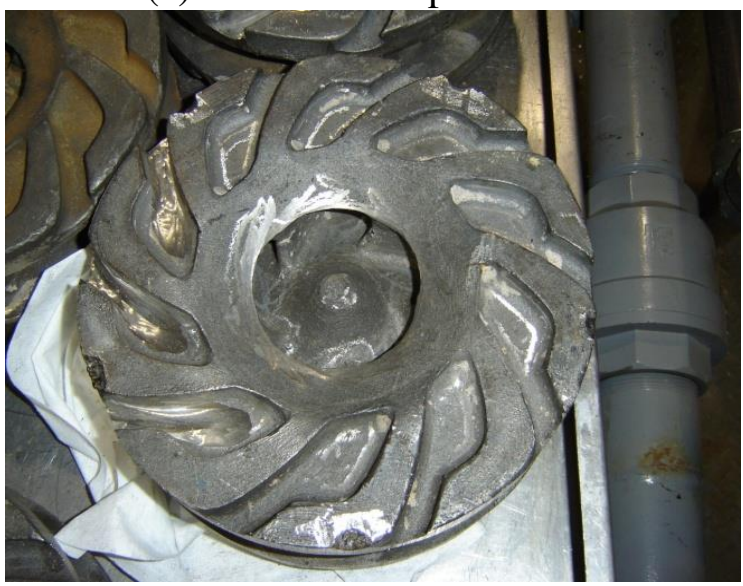

(c) S3 impeller edge damage

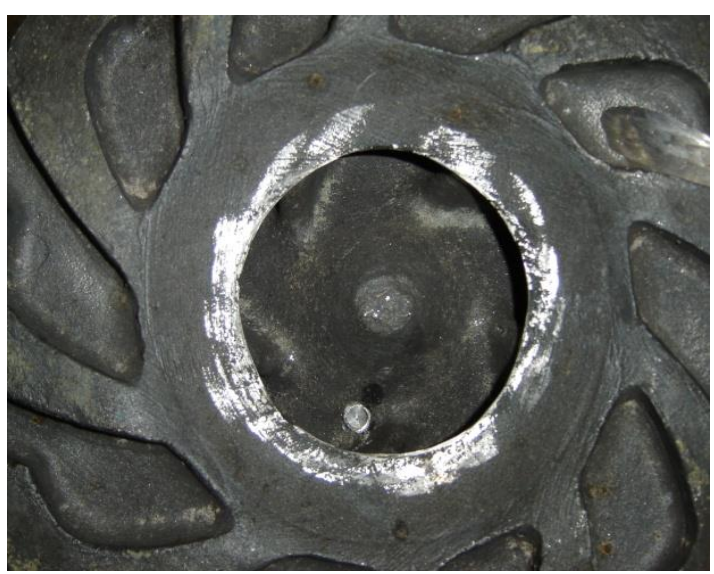

(b) S2 impeller perforation

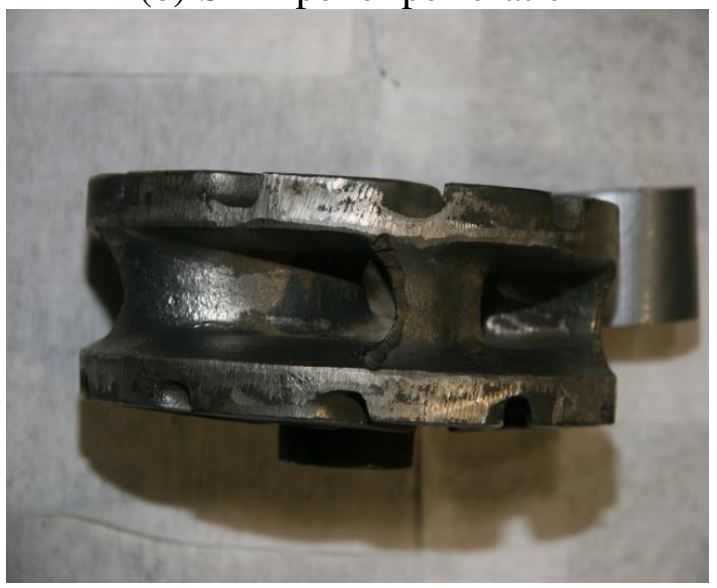

(d) S4 blade damage

Figure 13. Four failure modes of the impeller in the experiment

In the experiment, different impellers were replaced to collect the vibration signals of the centrifugal pump under different operation conditions. The steps of the whole experiment are 
summarized as follows:

(1) Establish the experimental device according to the schematic diagram of slurry pump test system shown in Figure 11. The normal impeller shown in Figure 13 is used as the impeller of the pump and sediment is added as the pumping medium. After starting the motor, we adjust the motor speed to $1200 \mathrm{rpm}, 1600 \mathrm{rpm}, 1800 \mathrm{rpm}, 2200 \mathrm{rpm}, 2600 \mathrm{rpm}$ through the known voltage, motor power, motor efficiency and other coefficients and instructions. According to the sampling time of 20 s and sampling frequency of $9009 \mathrm{HZ}$ shown in the previous article, NI LabView 7.0 application software and NI DAQ signal acquisition system were run to collect the three sets of three-dimensional vibration signals of the corresponding pump.

(2) The normal impeller in the original centrifugal pump is replaced by the impeller perforation of the fault S2 in Figure 13, and the other parts remain unchanged. Follow the previous steps to start the centrifugal pump and collect data. When a set of data is collected, the speed is set to 1400rpm, $1600 \mathrm{rpm}, \ldots, 2600 \mathrm{rpm}$ and the above steps are repeated to collect data.

(3) The S3 of impeller edge damage in Figure 13 (c) is selected to replace the impeller of S2 in the original centrifugal pump and other parts remain unchanged. Similarly, follow the previous steps to start the centrifugal pump and collect data.

(4) The S4 of blade damage in Figure 13 (d) is selected to replace the impeller of S3 in the original centrifugal pump and other parts remain unchanged. Similarly, follow the previous steps to start the centrifugal pump and collect data.

(5) After the experiment, the outlet valve of the pump was closed. Close the inlet valve after turning off the motor. Store experimental data to prepare for subsequent vibration signal analysis.

\section{Results and discussion}

\subsection{Multi-source dynamic feature extraction based on parallel factorization}

This multi-scale parallel factorization method for the extraction of characteristic signals in nonlinear multi-source and multi-fault modes is proposed in the article. Parallel factorization can not only perform high-dimensional data processing, but also has the uniqueness of the decomposition. This property makes the results of parallel factorization more realistic and has specific physical meanings. The third-order tensor constructed by multi-channel vibration signals through continuous wavelet transform is decomposed into the series of different modes of channel/frequency/time by the multi-scale parallel factor analysis algorithm. The spatial information is introduced into the time-frequency analysis of signals to form the three-dimensional spatial/time/frequency characteristic analysis of each factor. The simulation results show that the parallel factor decomposition for the high-dimensional data has the compatibility of decomposition path and overall consistency. As a result that the topographic map, spectrum and time contour of the multi-source fault signal in the centrifugal pump experiment are acquired. The multi-scale parallel decomposition method for extracting multi-source feature signals of nonlinear failure modes is applied in the fault diagnosis of centrifugal pumps. It analyzes the internal connection between the optimal decomposition paths of multi-source signal feature factors. The optimal non-linear correspondence relationship between failure modes and characteristic signals in time, frequency and space are constructed. Based on the correspondence and overall consistency of multi-source feature factor decomposition paths, we remodeled three-dimensional fault feature models such as the frequency spectrum and time profile of the fault feature factors, successfully extracted nonlinear multi-dimensional dynamic fault feature signals. Finally, the corresponding fluctuation regularities of the homologous non-linear failure mode in the multi-source signals were displayed. 


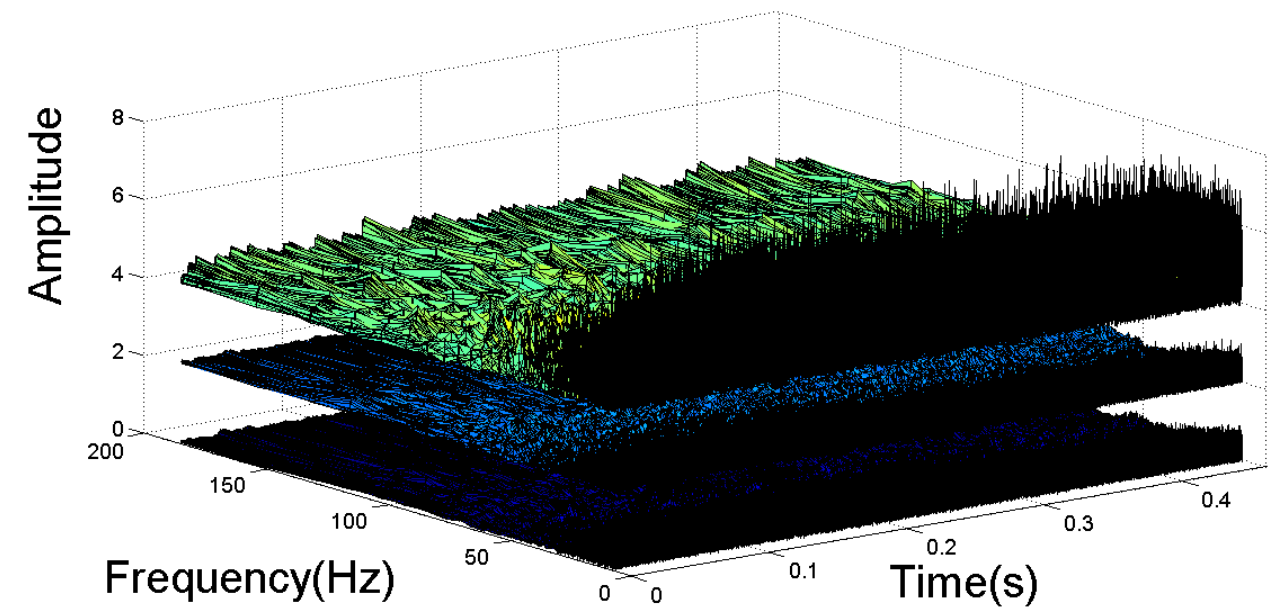

Figure 14. Time-frequency diagram of 3 vibration signals in normal state S1 mode 1

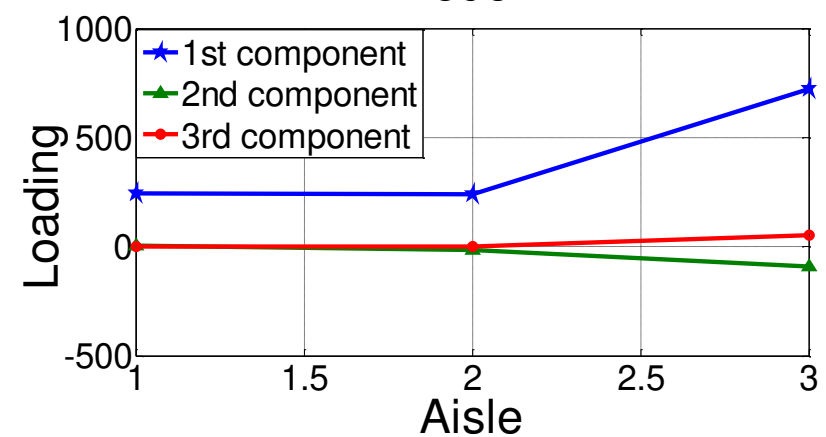

mode 3

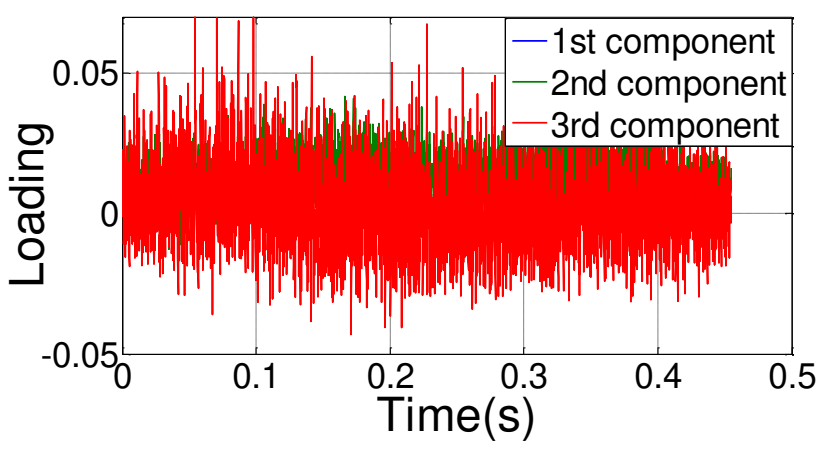

mode 2
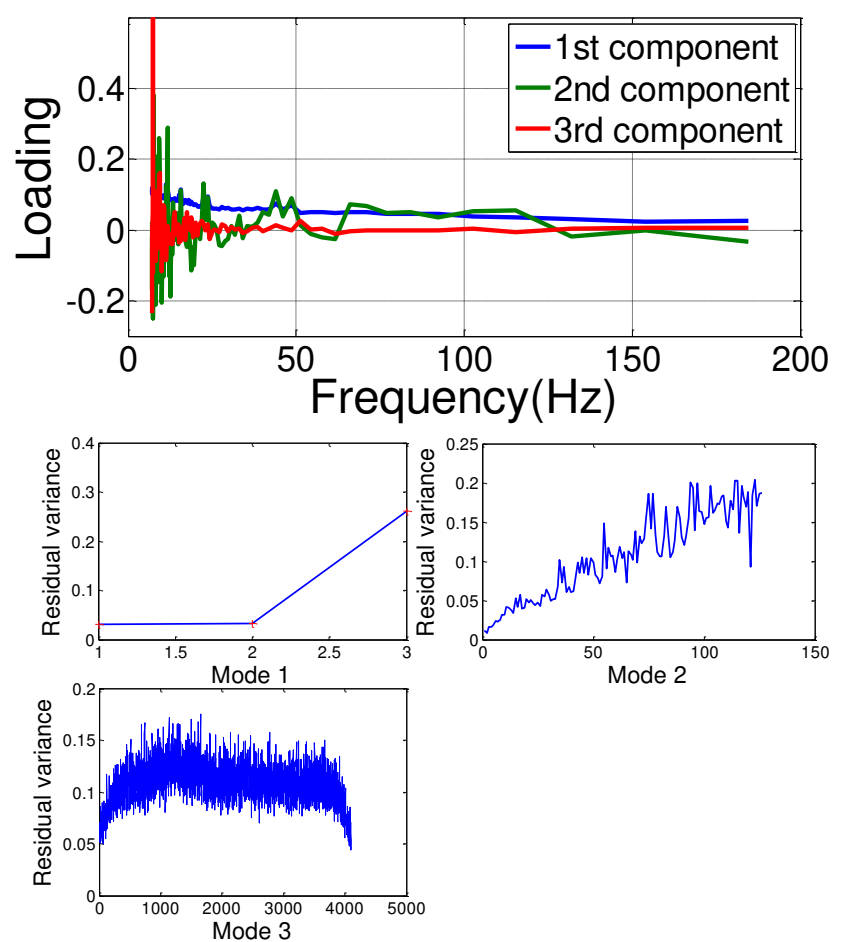

Figure 15. Parallel factor analysis when the centrifugal pump in normal state S1

Figure 14 shows the time-frequency diagram obtained of the vibration signals collected in the $\mathrm{X}$-axis direction of the three vibration signal collection points by continuous wavelet transformation when the slurry pump is in normal operation. Figure 15 shows the result of the parallel factor analysis of vibration signal of slurry pump after continuous wavelet transform in normal state. In this experiment, three-dimensional vibration sensors are set up at three measuring points. We analyze the vibration signals of these three measuring points to explore the three-dimensional spatial distribution and characteristic propagation path of dynamic characteristics on the mechanical structure of the slurry pump. Three groups of original vibration signals are transformed by continuous wavelet to obtain three-dimensional time-frequency signals to construct a third-order matrix. After multi-scale parallel factor analysis for the third-order tensor, the loading values and residual variance of the aisle, time and frequency factors are obtained. 


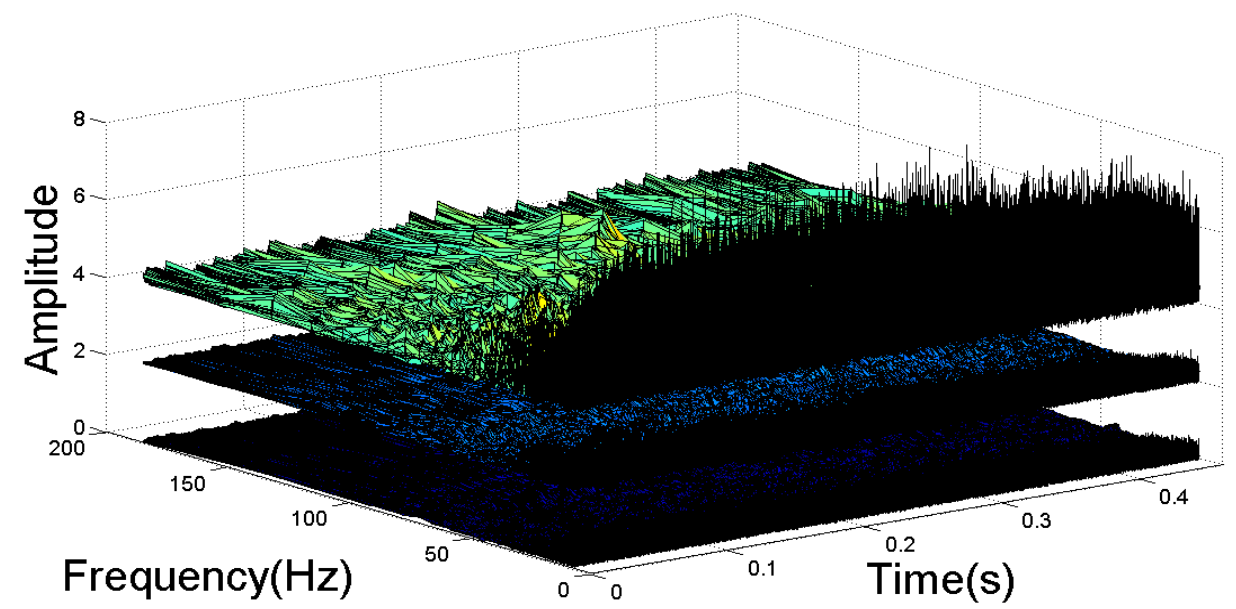

Figure 16. Time-frequency diagrams of 3 vibration signals under fault state S2

mode 1

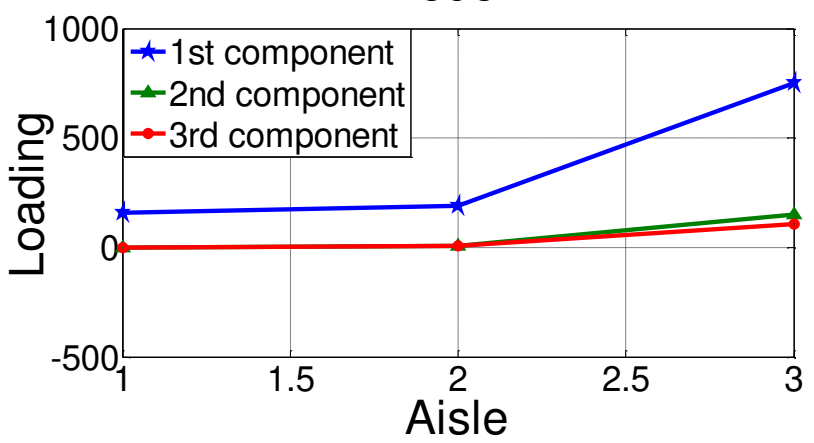

mode 3

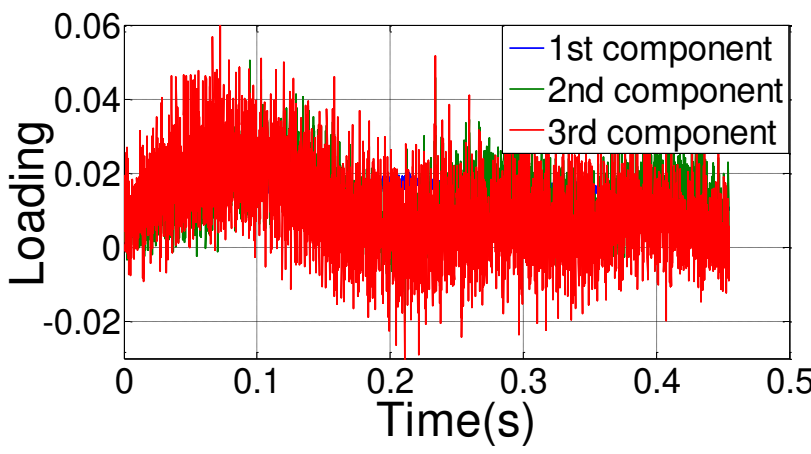

mode 2
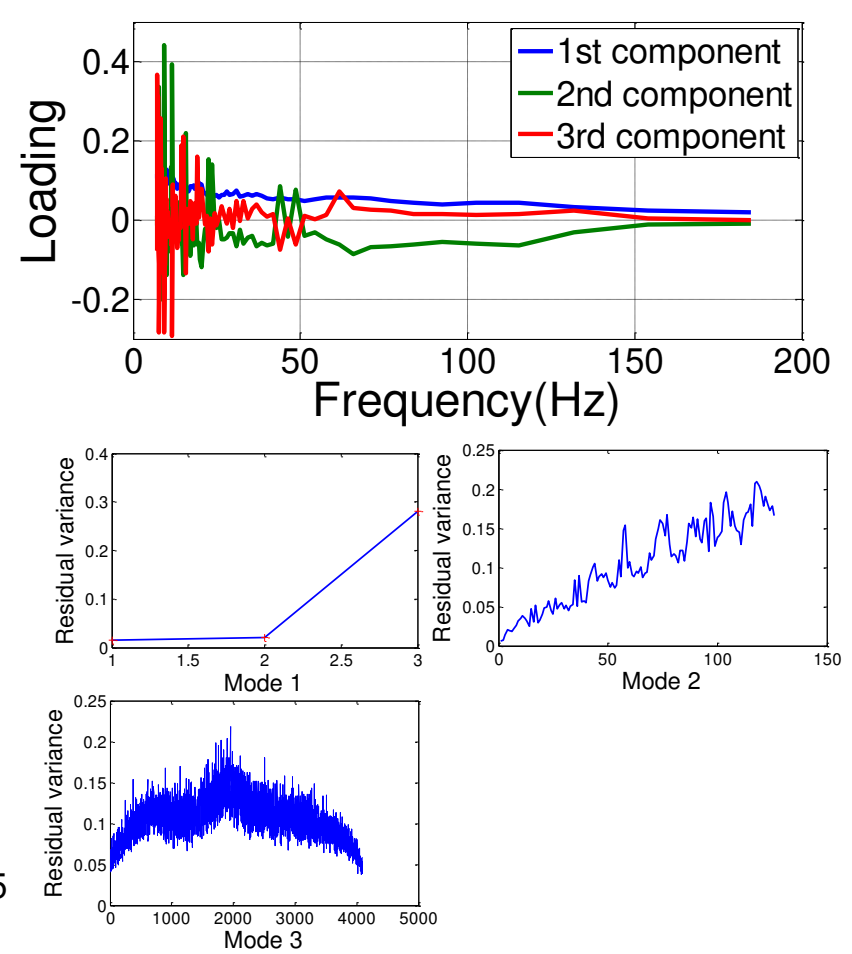

Figure 17. Parallel factor analysis when the centrifugal pump fails state S2

Figure 16 shows the time-frequency diagram of the vibration signals collected in the $\mathrm{X}$-axis direction of the three vibration signal collection points by continuous wavelet transformation when the slurry pump is in S2 impeller perforation. In the S2 state, the third-order tensor of 3*126*4096 is constructed by continuous wavelet transform. Figure 17 indicates the result of the loading values and residual variance of the aisle, time and frequency modes by the parallel factor analysis for the third-order tensor of slurry pump in state $\mathrm{S} 2$. 


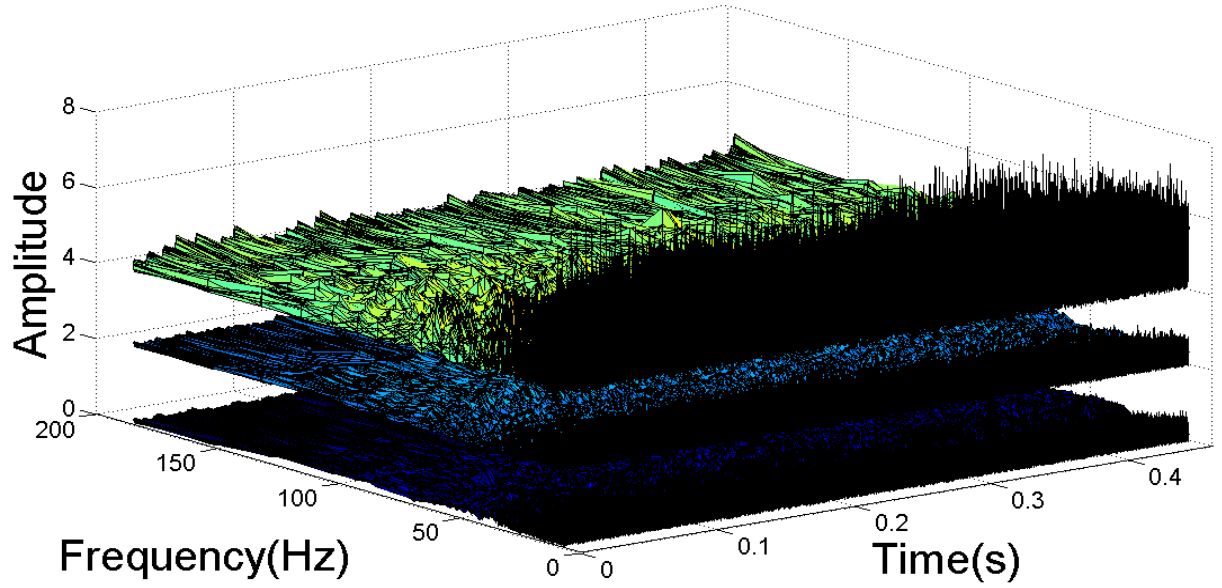

Figure 18. Time-frequency diagrams of 3 vibration signals under fault state $\mathrm{S} 3$

mode 1

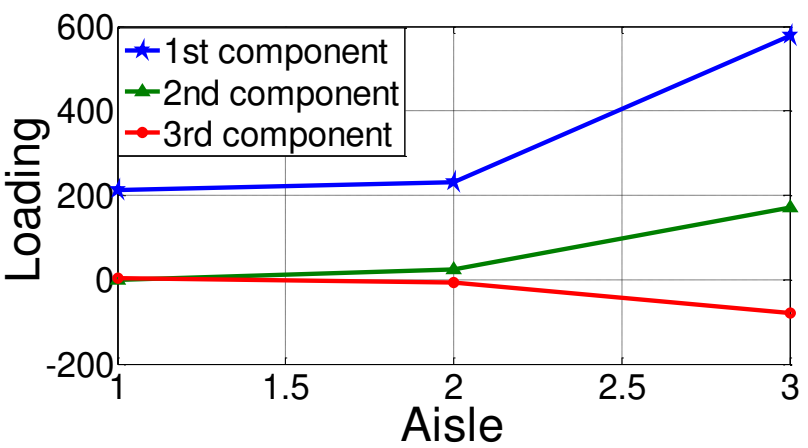

mode 3

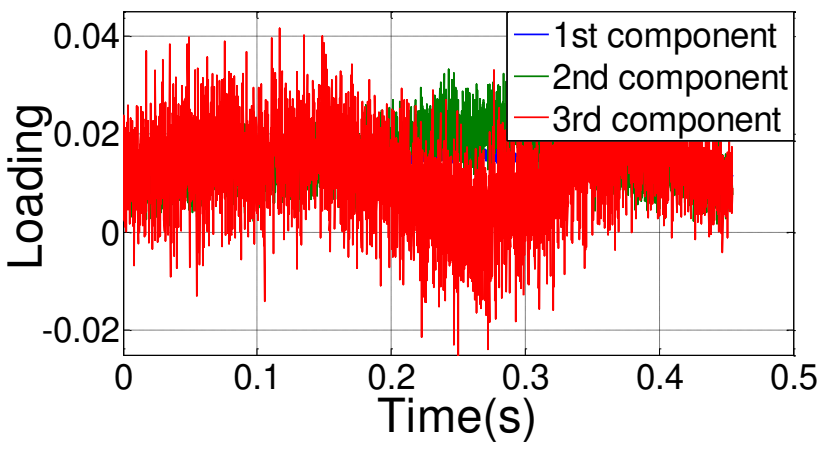

mode 2
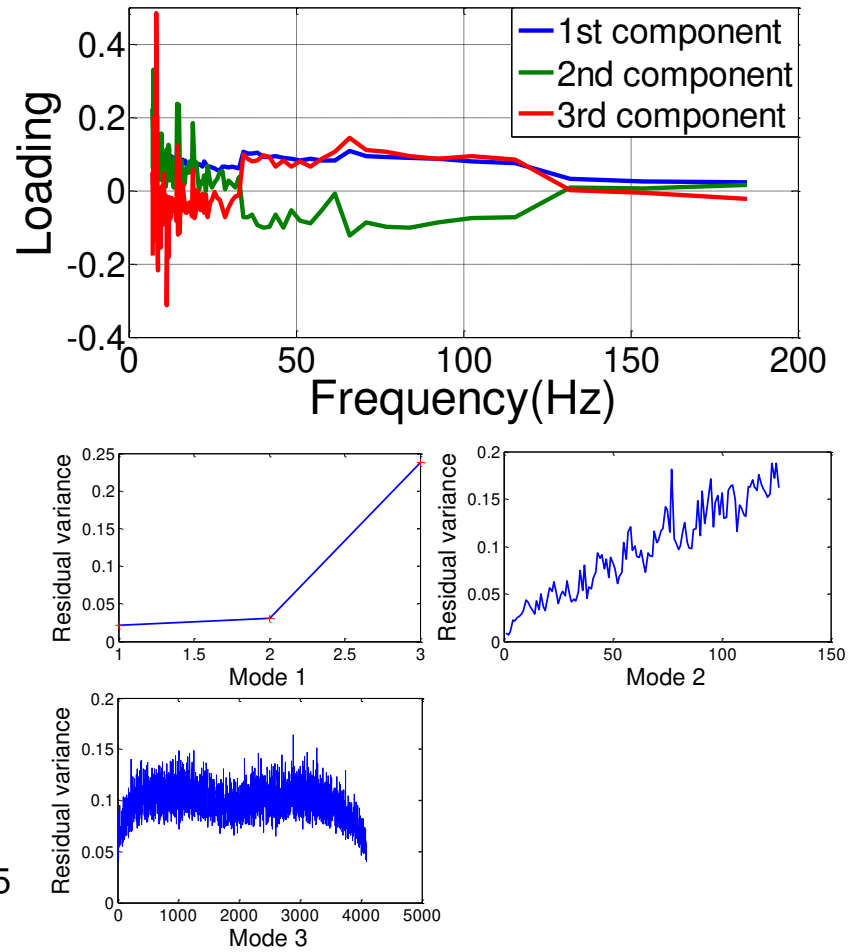

Figure 19. Parallel factor analysis when the centrifugal pump fails state S3

Figure 18 shows the time-frequency diagram of the vibration signals collected in the $\mathrm{X}$-axis direction of the three vibration signal collection points by continuous wavelet transformation when the slurry pump is in S3 impeller edge damage. In the S3 state, the third-order tensor of $3 * 126 * 4096$ is constructed by continuous wavelet transform. Figure 19 indicates the result of the loading values and residual variance of the aisle, time and frequency modes by the parallel factor analysis for the third-order tensor of slurry pump in state S3. 


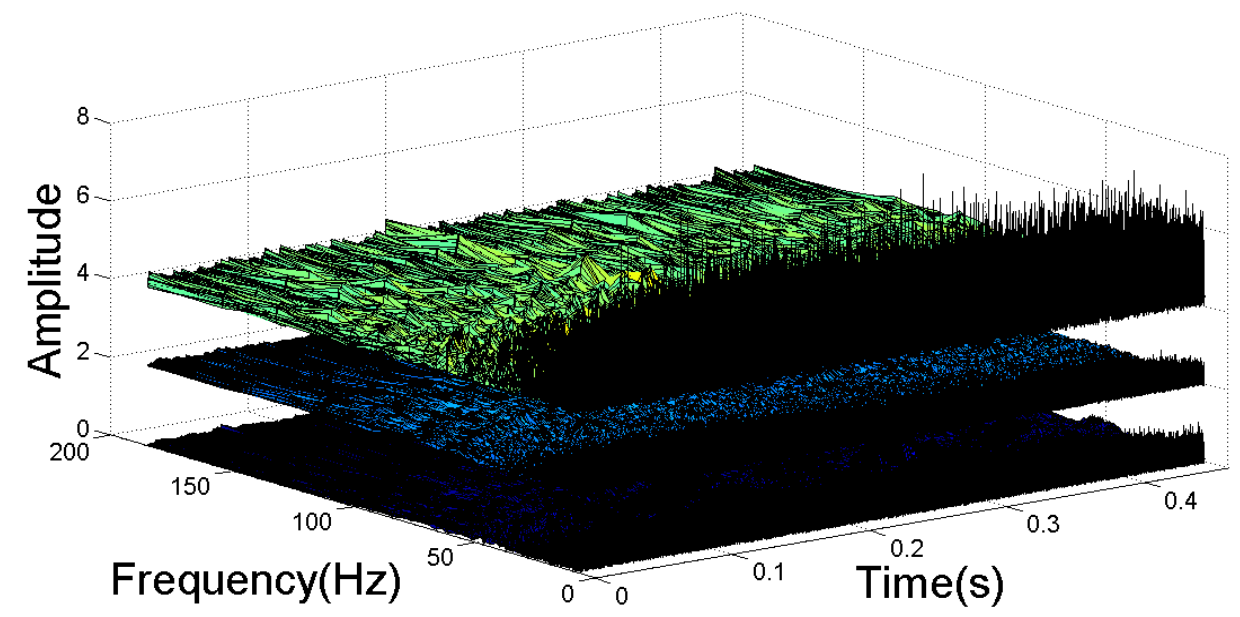

Figure 20. Time-frequency diagrams of 3 vibration signals under fault state S4 mode 1
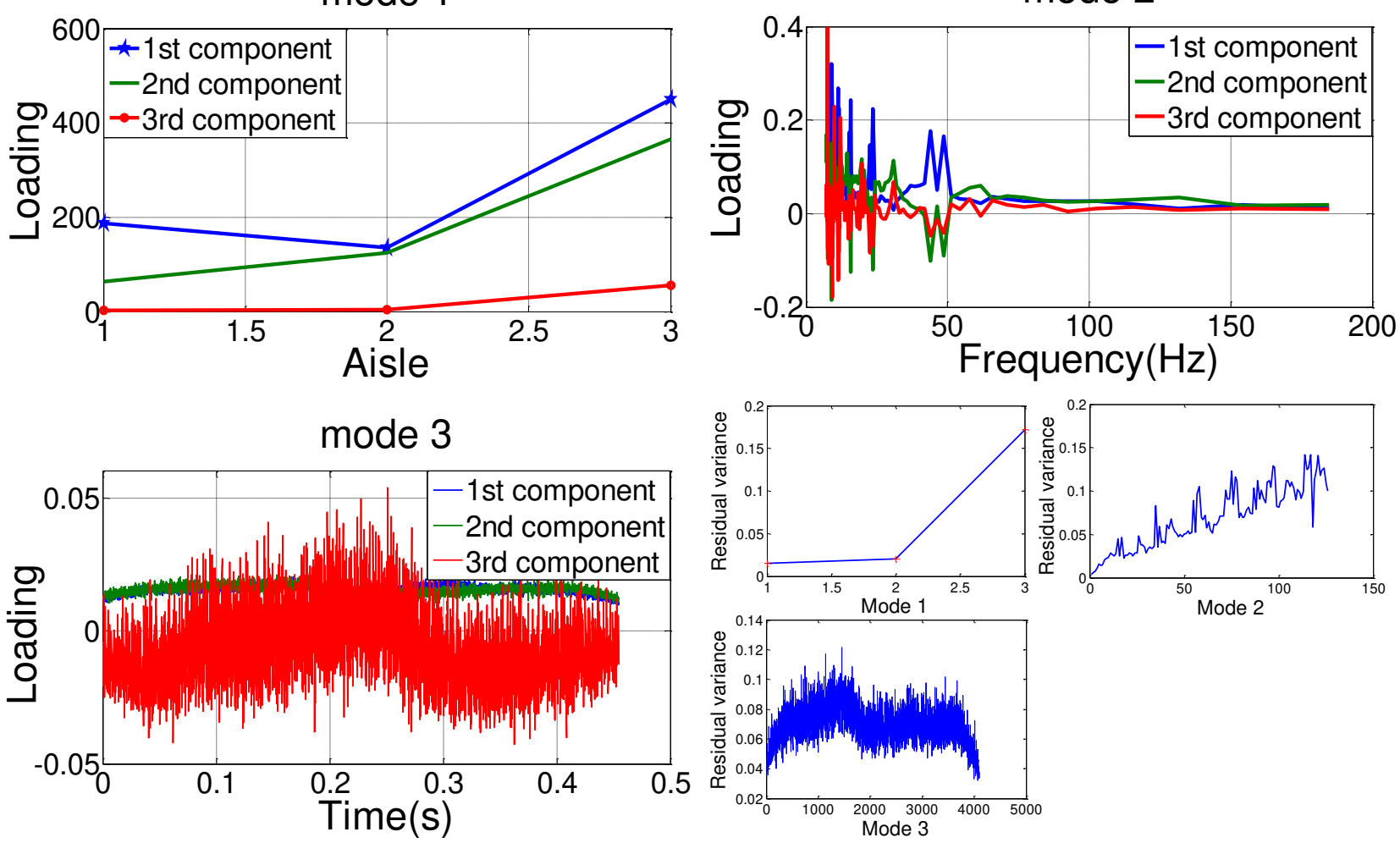

Figure 21. Parallel factor analysis when the centrifugal pump fails state S4

The operating state of the slurry pump system in our experimental device system has normal operation and 3 failure states. The failure states include impeller holes, leading edge damage and propeller blade damage. Similarly, Figure 20 shows the time-frequency diagram when the slurry pump is in S4 propeller blade damage. Figure 21 indicates the result of the loading values and residual variance of the aisle, time and frequency modes by the parallel factor analysis for state S4. We analyze the vibration signals of these three measuring points to discuss the three-dimensional spatial distribution and characteristic propagation path of dynamic characteristics on the mechanical structure of the slurry pump. By comparing the decomposition results of parallel factor analysis in the normal and fault state, there are obvious difference in the time loading factor and frequency loading factor component. Due to the phenomenon of characteristic coupling and aliasing of mechanical multi-source signals, the parallel factor analysis can optimize the independent characteristics of each channel on the surface of the mechanical structure and eliminate the mutual interference, overlap and redundancy of the characteristic signals between the channels. Therefore, the parallel factor analysis are effective in providing a basis for subsequent diagnosis of SPRT and the fault identification can be successfully implemented. 


\subsection{SPRT for the multi-source condition monitoring of centrifugal pump}

The proportions of the standard deviation " $\sigma$ " and mean " $\mu$ " of the test signal sequences have significant influence on the likelihood ratio in the sequential probabilistic ratio test. Therefore, the mean value and standard deviation of the frequency loading value after the parallel factor decomposition should be calculated first for the test signal sequence. Assuming the probability distribution of the frequency load value sequence of one set of signals under the multi-source condition monitoring of the centrifugal pump meets the null hypothesis $H_{i}: \mu=\mu_{i}$, and the probability distribution of the frequency load value sequence of the other set of signals satisfies the alternative hypothesis $H_{j}: \mu=\mu_{j}{ }^{[41]}$. Their corresponding standard deviation $\sigma$ remains unchanged. When the original hypothesis and the alternative hypothesis are both true, the joint probability density functions of these two sets of sequences are shown below.

$$
\begin{gathered}
P_{i k}\left(y_{k}\right)=\frac{1}{\sigma \sqrt{2 \pi}} \exp \left(-\frac{1}{2 \sigma^{2}}\left(y_{k}-\mu_{i}\right)^{2}\right) \\
P_{j k}\left(y_{k}\right)=\frac{1}{\sigma \sqrt{2 \pi}} \exp \left(-\frac{1}{2 \sigma^{2}}\left(y_{k}-\mu_{j}\right)^{2}\right)
\end{gathered}
$$

In formula (33), $P_{i k}\left(y_{k}\right)$ is the probability density function null hypothesis. $P_{j k}\left(y_{k}\right)$ in formula (34) is the probability density function under the alternative hypothesis. The SPRT probability ratio is calculated in formula (35).

$$
\lambda_{i, j}\left(Y_{S m}\right)=\frac{\prod_{k=1}^{n} P_{j k}}{\prod_{k=1}^{n} P_{i k}}=\frac{P_{j 1}\left(y_{1}\right) P_{j 2}\left(y_{2}\right) \Lambda P_{j k}\left(y_{k}\right)}{P_{i 1}\left(y_{1}\right) P_{i 2}\left(y_{2}\right) \Lambda P_{i k}\left(y_{k}\right)} \times \frac{P_{j 0}}{P_{i 0}}
$$

In order to make the calculation easier in practical applications, the likelihood ratio formula is further derived and simplified to obtain the formula (36). Where $Y_{S i}$ and $Y_{S j}$ are the to-be-checked sequences of vibration signals $S i$ and $S j$ respectively, $\Delta_{i, j}\left(Y_{S i}\right)$ and $\Delta_{i, j}\left(Y_{S j}\right)$ are the likelihood ratios of the sequence to be tested $Y_{S i}$ and $Y_{S j}$ respectively.

$$
\Delta_{i, j}\left(Y_{S m}\right)=\ln \lambda_{i, j}\left(Y_{S m}\right)=\ln \frac{\prod_{k=1}^{n} P_{j k}}{\prod_{k=1}^{n} P_{i k}}=\sum_{k=1}^{n} \ln \frac{P_{j k}}{P_{i k}} \quad m=i, j
$$

Referring to the sequential probability ratio test algorithm, we compare the likelihood ratio with the thresholds A and B to identify different forms of failure of the centrifugal pump. The size of A and $\mathrm{B}$ are closely related to the probability $\alpha$ of type I error and the probability $\beta$ of type II error. The variables $\alpha, \beta, \mathrm{A}$ and $\mathrm{B}$ are satisfied with the following relationship:

$$
\begin{aligned}
& a=\ln A=\ln \frac{1-\beta}{\alpha} \\
& b=\ln B=\ln \frac{\beta}{1-\alpha}
\end{aligned}
$$

For S1, S2, S3 and S4 four different impeller fault states of the centrifugal pump experimental system, Figure 22 shows the process of using the sequential probability ratio test algorithm to identify the fault . Vibration signals at three different positions collected under two different impeller fault conditions $(s j)$ and $(s j)$ are decomposed by parallel factors to obtain frequency loading values, which are calculated according to formulas (33)-(36) to obtain the likelihood ratio 
$\Delta_{i, j}$ of the sequential probability ratio test . The process of centrifugal pump fault identification is shown below. (1) If $\Delta_{i, j}=\ln \left(\lambda_{i, j}\right) \in(-\infty, b]$, accept $H_{j}$, the centrifugal pump system is under the condition $(s j)$. (2) If $\Delta_{i, j}=\ln \left(\lambda_{i, j}\right) \in[a, \infty)$, accept $H_{i}$, the centrifugal pump is under the condition $(s i)$. (3) If $\Delta_{\mathrm{i}, \mathrm{j}} \in[a, b]$, The likelihood ratio of sequential probabilistic ratio test continues to be calculated by extracting the next data in the test sequence according to formulas (33)-(36). The likelihood ratio will continue to be compared with the threshold value until the condition (1) or (2) is met or the number of iterations is reached. After the test is stopped and the probability parameters $\lambda_{1,2}\left(Y_{S 1}\right), \lambda_{1,2}\left(Y_{S 2}\right), \lambda_{1,3}\left(Y_{S 1}\right), \lambda_{1,3}\left(Y_{S 3}\right), \lambda_{1,4}\left(Y_{S 1}\right)$ and $\lambda_{1,4}\left(Y_{S 4}\right)$ are obtained, the conditions of centrifugal pump will be distinguished.

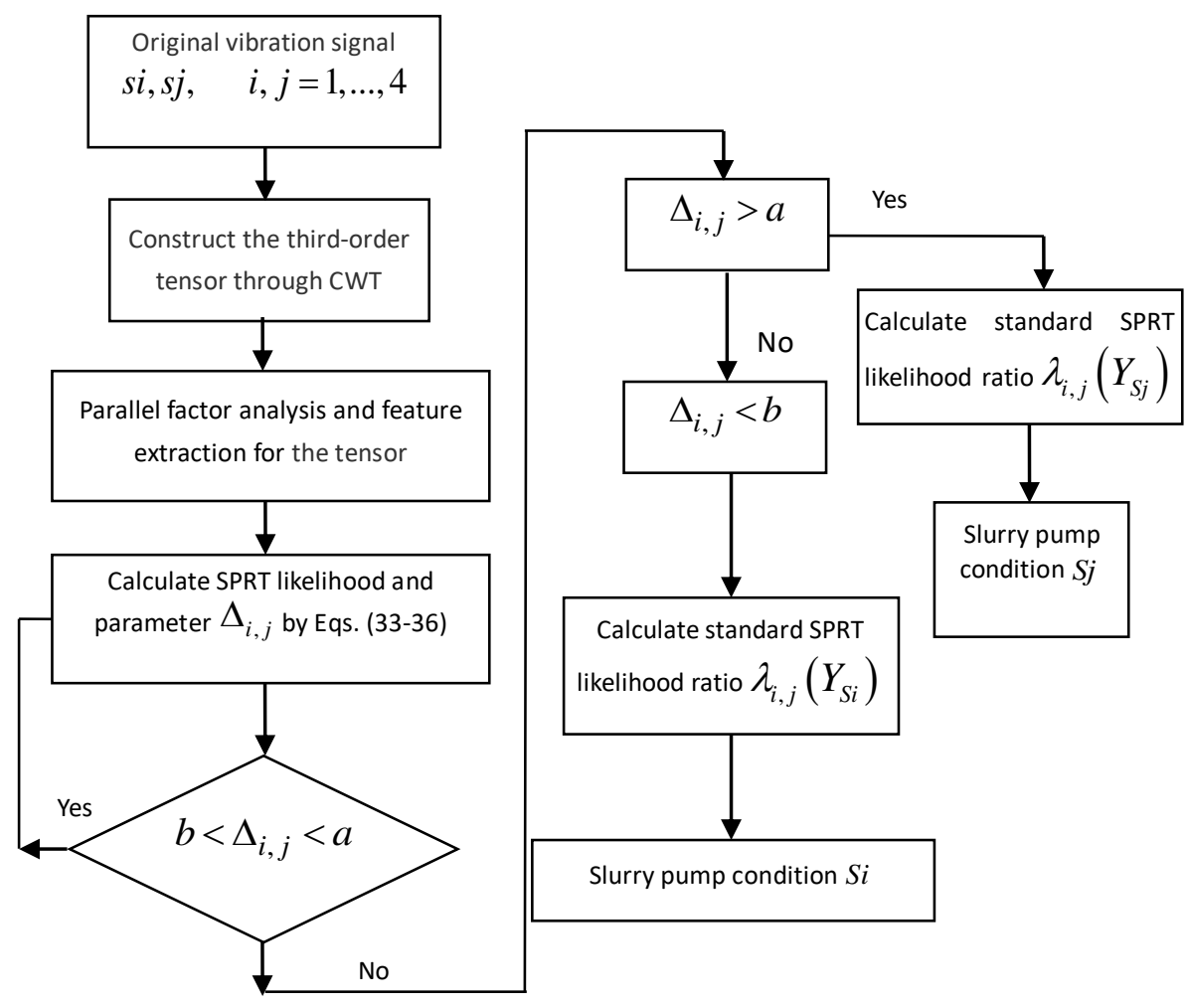

Fig. 22.Diagnostic process based on binary SPRT for centrifugal pump

The means of the signal $S 1, S 2, S 3$ and $S 4$ under the four conditions parameters are $\mu_{1}, \mu_{2}, \mu_{3}, \mu_{4}$. Then, the likelihood ratio is calculated and analyzed according to formulas (33) (36).SPRT probability ratios $\lambda_{i, j}\left(Y_{S i}\right)$ and $\lambda_{i, j}\left(Y_{S j}\right)$ are calculated by importing the testing data $\left(Y_{S i}, Y_{S j}\right)$ of the signal waveform for slurry pump $S i$ and $S j$ conditions to equation (35). Compare the likelihood ratios $\Delta_{i, j}\left(Y_{S i}\right)$ and $\Delta_{i, j}\left(Y_{S j}\right)$ with the threshold to determine the state $\mathrm{Si}$ and $\mathrm{Sj}$ of the centrifugal pump.

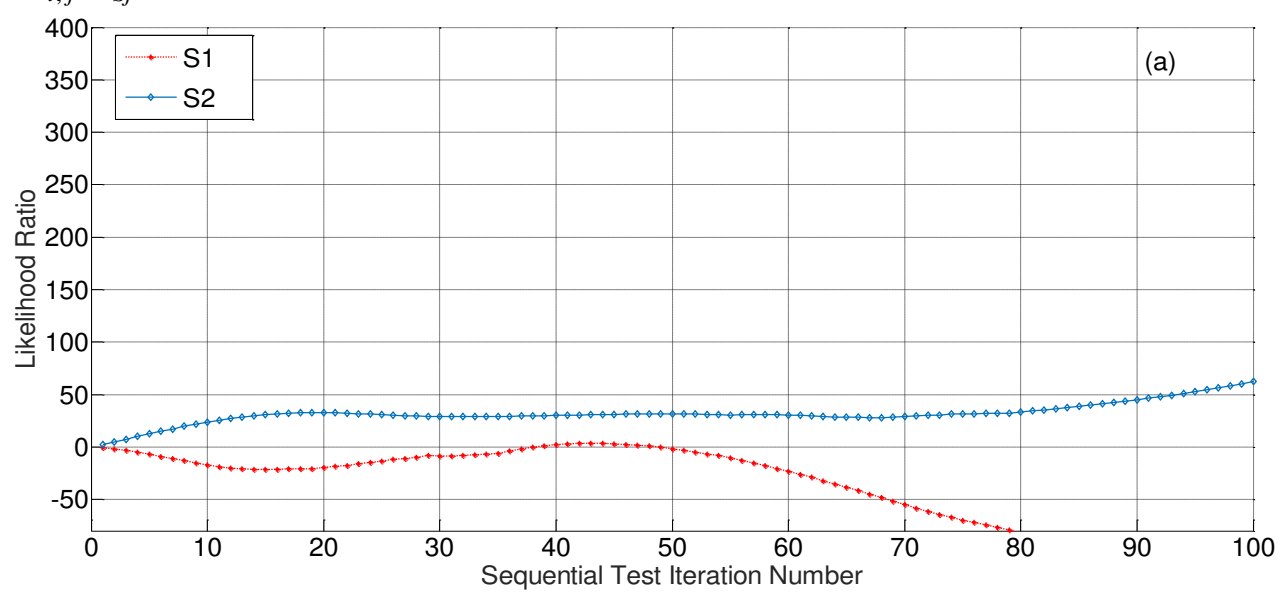



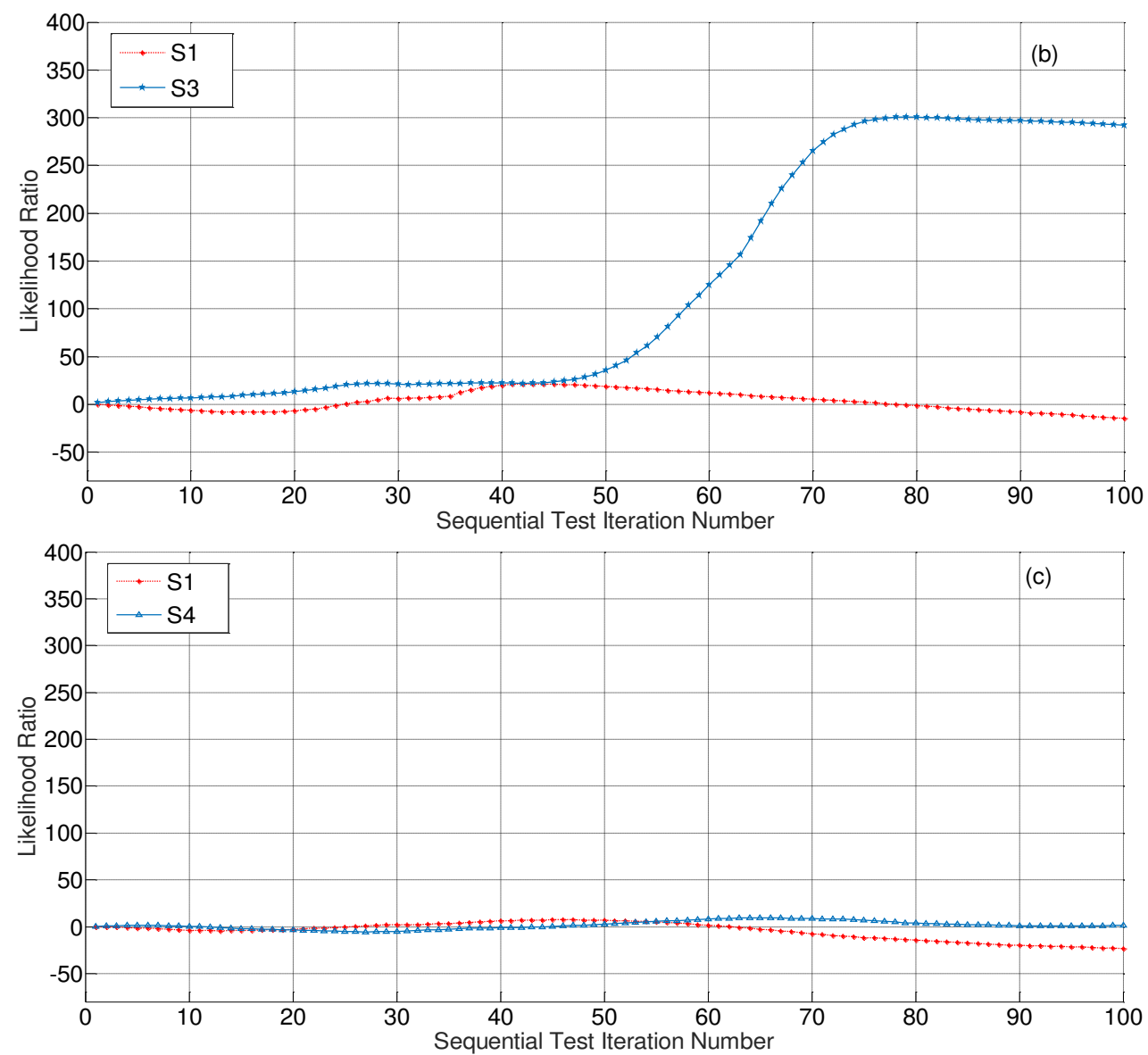

Fig. 23. Result of SPRT in variables $\lambda_{1,2}, \lambda_{1,3}, \lambda_{1,4}$ with calculation iteration

The difference in the variables $\lambda_{i, j}\left(Y_{S i}\right)$ and $\lambda_{i, j}\left(Y_{S j}\right)$ is used to distinguish different condition $(S i, S j)$ of the centrifugal pump. When the iteration periods are determined, the condition $S 1$ is compared with $S 2, S 3, S 4$ in the relation between SPRT probability ratios. Figure 23(a) shows the fluctuation curve between the likelihood ratios $\Delta_{1,2}\left(Y_{S 1}\right)$ and $\Delta_{1,2}\left(Y_{S 2}\right)$ of the signals S1 and S2 during the determined number of iteration. It can be seen from the curve in Figure 23(a) that $\Delta_{1,2}\left(Y_{S 1}\right)<b$ displays that the centrifugal pump is in the normal state of $\mathrm{S} 1 ; \Delta_{1,2}\left(Y_{S 2}\right)>a$ indicates that the centrifugal pump is in a fault state of the S2 impeller perforation. From the curve in Figure 23(b), it can be seen that $\Delta_{1,3}\left(Y_{S 1}\right)<b$ indexes that the centrifugal pump is in the normal state of $\mathrm{S} 1 ; \Delta_{1,3}\left(Y_{S 3}\right)>a$ indicates that the centrifugal pump is in the fault state of S3 impeller edge damage. In Fig. 23(c), the inequality $\left(\Delta_{1,4}\left(Y_{S 1}\right)\right)<_{b}$ is satisfied, the condition is $\mathrm{S} 1$. The inequality $\left(\Delta_{1,4}\left(Y_{S 4}\right)\right)>a$ is satisfied, the condition of the centrifugal pump is S4 impeller blade damage. The SPRT parameters $\Delta_{1, m}, m=2,3,4$ in Fig. 23(a-c) are adopted to distinguish that the normal condition (S1) of the centrifugal pump from the conditions(S2,S3,S4). 

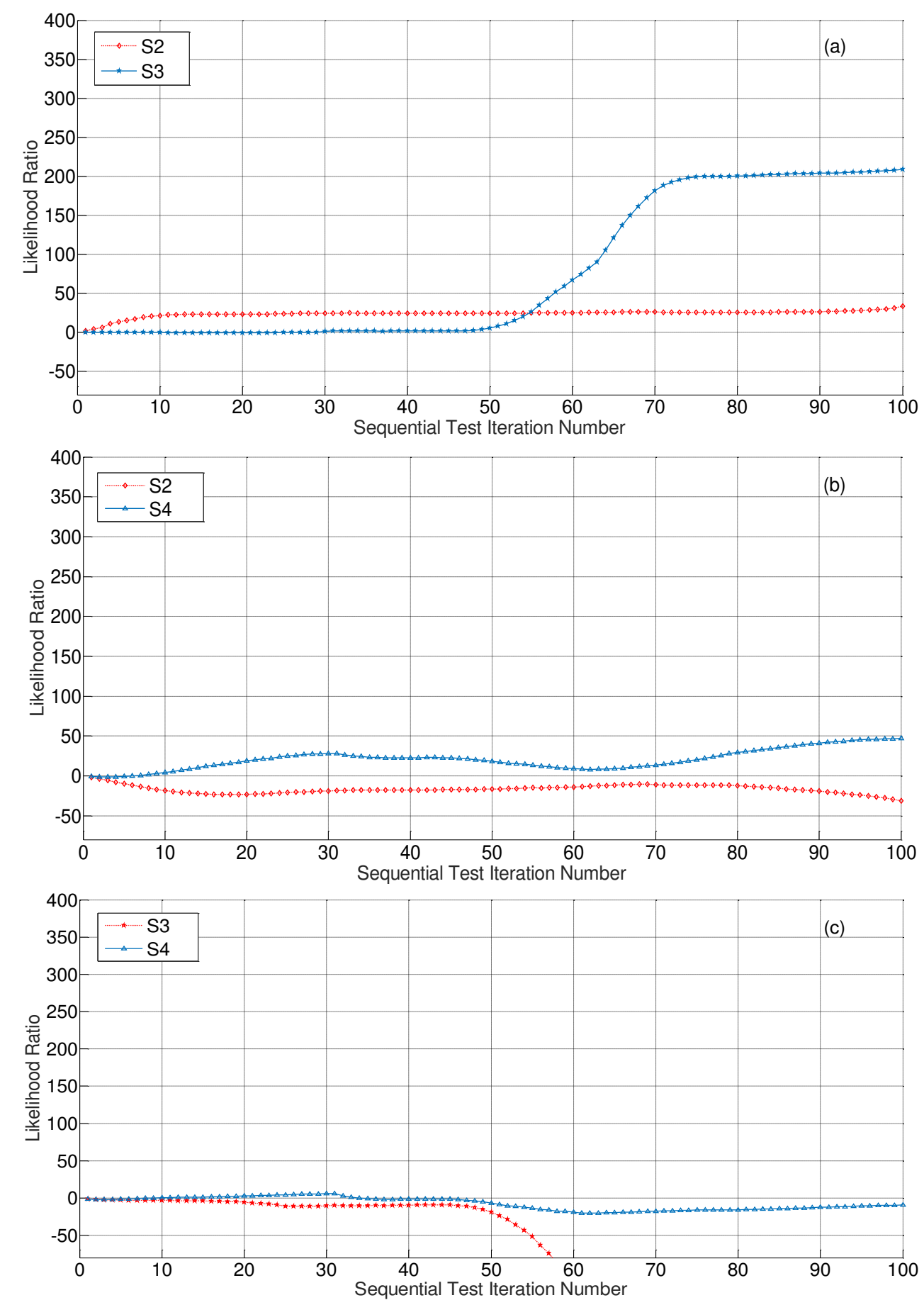

Fig. 24. Result of SPRT in variables $\lambda_{2,3}, \lambda_{2,4}, \lambda_{3,4}$ with calculation cycles

We found that the different fault states of the centrifugal pump in the experiment can also be distinguished by this method of SPRT. Fig. 24(a) indicates the SPRT parameters $\lambda_{2,3}$ in Eqs. (33-36) of the testing sequences $Y_{S 2}$ and $Y_{S 3}$. The SPRT inequality $\left(\Delta_{2,3}\left(Y_{S 2}\right)\right)<b$ is satisfied, then the condition of the centrifugal pump is $\mathrm{S} 2$. The inequality $\left(\Delta_{2,3}\left(Y_{S 3}\right)\right)>a$ is satisfied, then the condition of the slurry pump is S3(impeller edge damage). It can be seen from the curve in Figure 24(b) that when $\left(\Delta_{2,4}\left(Y_{S 2}\right)\right)<b$ is satisfied, the centrifugal pump is fault $\mathrm{S} 2$ (impeller perforation); when $\left(\Delta_{2,4}\left(Y_{S 4}\right)\right)>a$ is satisfied, the centrifugal pump is fault S4 (impeller blade damage). When the inequality $\left(\Delta_{3,4}\left(Y_{S 3}\right)\right)<b$ is satisfied in Fig. 24(c), the condition is S3 (impeller edge damage). When $\left(\Delta_{3,4}\left(Y_{S 4}\right)\right)>a$ is satisfied, the condition of the centrifugal pump is $\mathrm{S} 4$ (impeller blade damage). The 
parameters $\left(\lambda_{i, j}\left(Y_{S i}\right), \lambda_{i, j}\left(Y_{S j}\right)\right)$ are effective indicator to monitor the different conditions of the multi-fault and multi-source centrifugal pump.

\section{Conclusion}

This multi-scale parallel factorization method for the extraction of characteristic signals in nonlinear multi-source and multi-fault modes is proposed in the article. Parallel factorization can not only perform high-dimensional data processing, but also has the uniqueness of the decomposition. This property makes the results of parallel factorization more realistic and has specific physical meanings. First, we introduced the basic mathematical properties related to parallel factor analysis: matrix essential equalization, recognizability and uniqueness of parallel factorization, parallel factor model and its solution algorithm of trilinear alternating least squares (TALS), etc. Then, through numerical simulation, the parallel factorization is used to explore the non-linear correspondence relationship of multi-source signal characteristic factors in time, frequency and space under different simulation states. By adjusting the different frequency, time, phase and amplitude of the analog signal, the loading values of the three modes are captured after parallel factorization to research the corresponding relationship between it and the analog signal. Finally, the parallel factor analysis is applied to the centrifugal pump fault diagnosis experimental system to analyze the state characteristics under multiple fault modes. The nonlinear multi-dimensional actional fault characteristic parameter of the impellers with different faults of the centrifugal pump were triumphantly acquired and the corresponding fluctuation regularities of the homologous fault mode characteristics in the multi-source signals were displayed.

The analysis of the comprehensive result graph shows that the centrifugal pump fault diagnosis methodology based on Parallel Factor Analysis of multiple scales and Sequential Probability Ratio Test is effective and reliable. This method first analyzes the collected vibration signals through parallel factor analysis and then conducts sequential probability ratio testing. It identifies different failure modes by comparing likelihood ratios and thresholds. Not only normal conditions and fault conditions can be identified, but also different fault conditions can be distinguished. Therefore, the methodology proposed in this contents of article is very suitable for nonlinear multi-source and multi-fault signal analysis and processing.

\section{Acknowledgements}

The Reliability Research Lab in the Department of Mechanical Engineering at the University of Alberta in Canada provided the original experimental data. The National Natural Science Foundation of China (Grant No. 51775390, 51901164, 51805378), the Natural Science Foundation of Hubei Province (Grant No. 2018CFB394), and the Foundation of Wuhan Science and Technology Bureau (Grant No. 2019010701011417) provides the financial support for paper research.

\section{Abbreviations}

PARAFAC: Parallel factor analysis; SPRT: Sequential probability ratio test; CNN: Convolutional neural network; EMD: Empirical mode decomposition; VMD: Variational mode decomposition; EEG: Electroencephalogram; ANN-MLP: artificial neural network-multilayer perceptron; TALS: Trilinear alternate least squares; LS: Least squares; PDF: Probability density function.

\section{Authors' contributions}

The algorithms proposed in this paper have been conceived by all authors. Hanxin Chen designed and performed the experiments and then analyzed the results. Liu Yang performed the experiments and analyzed the simulation results. Liu Yang wrote the paper. All authors read and approved the final manuscript.

\section{Funding}

This research was funded by the National Natural Science Foundation of China (Grant No. 51775390, 51901164, 51805378), the Natural Science Foundation of Hubei Province (Grant No. 2018CFB394), and the Foundation of Wuhan Science and Technology Bureau (Grant No. 2019010701011417). 


\section{Availability of data and materials}

All data generated or analyzed during this study are included in this published article.

\section{Ethics approval and consent to participate}

Not applicable.

\section{Consent for publication}

Not applicable.

\section{Competing interests}

The authors declare that they have no competing interests.

\section{References}

[1] Azizi R, Attaran B, Hajnayeb A, Ghanbarzadeh A, Changizian M. Improving accuracy of cavitation severity detection in centrifugal pumps using a hybrid feature selection technique. Measurement, 2017; 108: 9-17.

[2] Shankar, V. K. Arun; Subramaniam, Umashankar; Padmanaban, Sanjeevikumar; et al. Experimental Investigation of Power Signatures for Cavitation and Water Hammer in an Industrial Parallel Pumping System. ENERGIES, 2019, 12(7).

[3] Hanxin Chen, Dongliang Fan, Jinmin Huang, Wenjian Huang, Guangyu Zhang, and Lang Huang,Finite element analysis model on ultrasonic phased array technique for material defect time of flight diffraction detection. Science of advanced material, 2020, 12(5), 665-675.

[4] Jiang, Fei; Ding, Kang; He, Guolin; et al, Sparse dictionary design based on edited cepstrum and its application in rolling bearing fault diagnosis. JOURNAL OF SOUND AND VIBRATION, 2021, Vol.490.

[5] Janssens, Olivier; Slavkovikj, Viktor; Vervisch, Bram; et al, Convolutional Neural Network Based Fault Detection for Rotating Machinery. JOURNAL OF SOUND AND VIBRATION, 2016, Vol.377:331-345.

[6] Ben Ali, Jaouher; Fnaiech, Nader; Saidi, Lotfi; et al,. Application of empirical mode decomposition and artificial neural network for automatic bearing fault diagnosis based on vibration signals. APPLIED ACOUSTICS, 2015, Vol.89: 16-27.

[7] Mousmoulis G, Karlsen-Davies N, Aggidis G, Anagnostopoulos I, Papantonis D. Experimental analysis of cavitation in a centrifugal pump using acoustic emission, vibration measurements and flow visualization. Eur J Mech B Fluids 2019;75:300-11.

[8] Zhang M, Jiang Z, Feng K. Research on variational mode decomposition in rolling bearings fault diagnosis of the multistage centrifugal pump. Mech Syst Sig Process 2017;93:460-93.

[9] Hanxin Chen,Dong Liang Fan, Lu Fang, Wenjian Huang, Jinmin Huang, Chenghao Cao, Liu Yang, Yibin He and Li Zeng, Particle swarm optimization algorithm with mutation operator for particle filter noise reduction in mechanical fault diagnosis, International journal of pattern recognition and artificial intelligence, https://doi.org/10.1142/S0218001420580124

[10] Chen, H., Shang, Y., \& Sun, K. (2013). Multiple fault condition recognition of gearbox with sequential hypothesis test. Mechanical Systems and Signal Processing, 40(2), 469-482.

[11] Hanxin Chen, Wenjian Huang, Jinmin Huang, Chenghao Cao, Liu Yang, Yibin He and Li Zeng,Multi-fault condition monitoring of slurry pump with principle component analysis and sequential hypothesis test. International journal of pattern recognition and artificial intelligence, 2020, 34(7). 
[12] Kumar, A., Gandhi, C. P., Zhou, Y., Kumar, R., \& Xiang, J. (2020). Improved deep convolution neural network (CNN) for the identification of defects in the centrifugal pump using acoustic images. Applied Acoustics, 167, 107399. doi:10.1016/j. apacoust.2020.107399 .

[13] Azizi R, Attaran B, Hajnayeb A, Ghanbarzadeh A, Changizian M. Improving accuracy of cavitation severity detection in centrifugal pumps using a hybrid feature selection technique. Measurement 2017; 108:9-17.

[14] Liu Yang, Hanxin Chen, Fault diagnosis of gearbox based on RBF-PF and particle swarm optimization wavelet neural network. Neural computing and applications, 2019, 31(9), 4463-4478.

[15] Zhang M, Jiang Z, Feng K. Research on variational mode decomposition in rolling bearings fault diagnosis of the multistage centrifugal pump. Mech Syst Sig Process 2017; 93:460-93.

[16] Lu C, Wang S, Zhang C. Fault diagnosis of hydraulic piston pumps based on a two-step EMD method and fuzzy C-means clustering. Proc Instit Mech Eng,Part C 2016;230:2913-28.

[17]Hanxin Chen, Lu Fang, Dong Liang Fan, Wenjian Huang, Jinmin Huang, Chenghao Cao, Liu Yang, Yibin He and Li Zeng, Particle Swarm Optimization Algorithm with Mutation Operator for Particle Filter Noise Reduction in Mechanical Fault diagnosis, International Journal of Pattern Recognition and Artificial Intelligence, https://doi.org/10.1142/S0218001420580124.

[18] Hanxin Chen, Yongting Chen, Liu Yang, Intelligent early structural health prognosis with nonlinear system identification for RFID signal analysis.COMPUTER COMMUNICATIONS, 2020, 157:150-161.

[19] Chen,Hanxin; Huang Lang; Yang Liu; Chen Yongting; Huang Jinmin. Model-base method with nonlinear ultrasonic system identification for mechanical structural health. Transactions On Emerging Telecommunications Technologies, 2020, DOI:10.1002/ett.3955.

[20] Chen, Hanxin; Zhang, Guangyu; Fan, Dongliang; Fang, Lu; Huang, Lang. Nonlinear Lamb wave analysis for microdefect identification in mechanical structural health assessment. MEASUREMENT, 2020, 164.

[21] Carroll, J. D., \& Chang, J.-J. (1970). Analysis of individual differences in multidimensional scaling via an n-way generalization of "Eckart-Young" decomposition. Psychometrika, 35(3), 283-319.

[22] Harshman, R.A., 1970. Foundations of the PARAFAC procedure: models and conditions for an 'explanatory' multi-modal factor analysis. UCLA Work. Pap. Phon. 16, $1-84$.

[23] Chen Hanxin, Lu Yanjun, Tu Ling, Fault identification of gearbox degradation with optimized wavelet neural network, Shock and vibration, 2013, 20(2), 247-262.

[24] Zhao, Rui; Yan, Ruqiang; Chen, Zhenghua; et al, Deep learning and its applications to machine health monitoring. MECHANICAL SYSTEMS AND SIGNAL PROCESSING, 2019, Vol.115:213-237.

[25] Li Y, Cheng G, Liu C, Chen X. Study on planetary gear fault diagnosis based on variational mode decomposition and deep neural networks.Measurement 2018;130:94-104.

[26] Schmitz, S. Katharina; Hasselbach, Philipp P.; Ebisch, Boris; et al. Application of Parallel Factor Analysis (PARAFAC) to electrophysiological data. FRONTIERS IN NEUROINFORMATICS, 2015, 8.

[27] Miwakeichi, Fumikazu; Martinez-Montes, Eduardo,et al. Decomposing EEG data into space-time-frequency components using Parallel Factor Analysis.NEUROIMAGE, $2004,22(3), 1035-1045$. 
[28] Rost'akova, Zuzana; Rosipa, Roman; Seifpour, Saman, et al. A Comparison of Non-negative Tucker Decomposition and Parallel Factor Analysis for Identification and Measurement of Human EEG Rhythms . MEASUREMENT SCIENCE REVIEW, 2020, 20(3), 126-138.

[29] Choi, Ji Yeh;Hwang, Heungsun; Timmerman, Marieke E. Functional Parallel Factor An alysis for Functions of One- and Two-dimensional Arguments .PSYCHOMETRIKA, 20 $18,83(1), 1-20$.

[30] Wang, Jing; Wang, Juan ; Wan, You ;Li, Xiaoli. The Frontal area with higher Frequency response is the Principal Feature of laser-evoked Potentials in rats with chronic inflammatory Pain: a Parallel Factor analysis study. FRONTIERS IN NEUROLOGY, 2017, 8.

[31] Liu Yang,Hanxin Chen, Yao Ke, Lang Huang, Qi Wang, Yuzhuo Miao, Li Zeng. A novel time-frequency-space method with parallel factor theory for big data analysis in condition monitoring of complex system. International Journal of Advanced Robotic Systems, 2020, 17(2).

[32] Yang, Cheng;Jia, Minping.A novel weak fault signal detection approach for a rolling bearing using variational mode decomposition and phase space parallel factor analysis. MEASUREMENT SCIENCE AND TECHNOLOGY, 2019, 30(11).

[33]Luo, Lijia; Bao, Shiyi; Mao, Jianfeng; et al. Monitoring Batch Processes Using Sparse Parallel Factor Decomposition. Industrial \& Engineering Chemistry Research, 2017, 56(44), 12682-12692.

[34] Gu, Haiyang ; Liu, Kaiqi; Huang, Xingyi; et al. Feasibility study for the analysis of coconut water using fluorescence spectroscopy coupled with PARAFAC and SVM methods. BRITISH FOOD JOURNAL, 2020, DOI: 10.1108/BFJ-12-2019-0941.

[35] Han, Xiu; Yu, Huibin; Song, Yonghui; et al. Characterizing humic substances from a large-scale lake with irrigation return flows using 3DEEM-PARAFAC with CART and 2D-COS. JOURNAL OF SOILS AND SEDIMENTS,2020,20(9),3514-3523.

[36] Chetouani, Yahya. A sequential probability ratio test (SPRT) to detect changes and pro cess safety monitoring. PROCESS SAFETY AND ENVIRONMENTAL PROTECTION, 2014, 92(3), 206-214.

[37]Guo, Peng; Infield, David. Wind Turbine Power Curve Modeling and Monitoring With Gaussian Process and SPRT.IEEE TRANSACTIONS ON SUSTAINABLE ENERGY, 20 20, 11(1), 107-115.

[38] Wang, Rong; Xiong, Zhi; Liu, Jianye; et al. Chi-Square and SPRT Combined Fault Detection for Multisensor Navigation. IEEE TRANSACTIONS ON AEROSPACE AND ELECTRONIC SYSTEMS, 2016, 52(3), 1352-1365.

[39] Kasai, Hiroyuki. Fast online low-rank tensor subspace tracking by CP decomposition u sing recursive least squares from incomplete observations. NEUROCOMPUTING, 2019, $347,177-190$.

[40]Abraham Wald. Sequential Analysis.Professor of Mathematical Statistics Columbia University, 1984:37-42.

[41] Parras, Juan;Zazo, Santiago. Using one class SVM to counter intelligent attacks against an SPRT defense mechanism. AD HOC NETWORKS, 2019, 94. 
Figures
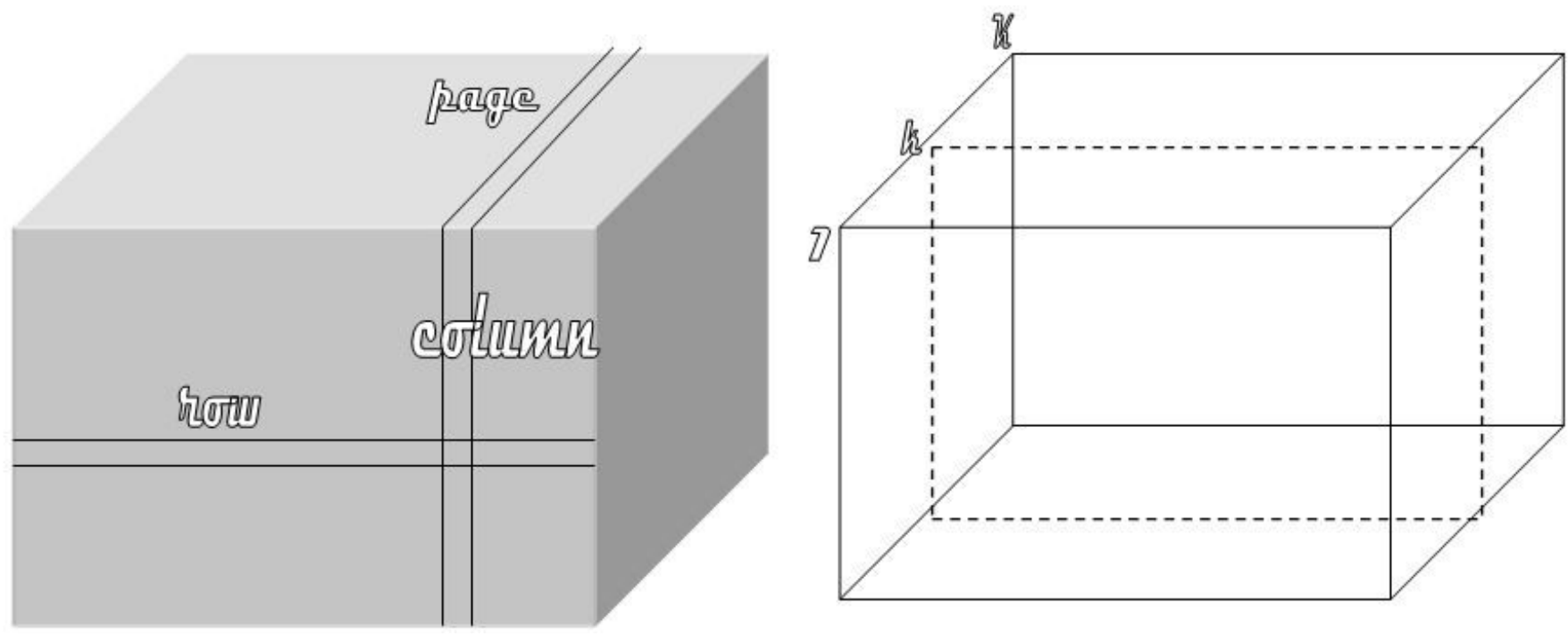

Figure 1

Three-dimensional matrix and its sub-matrix

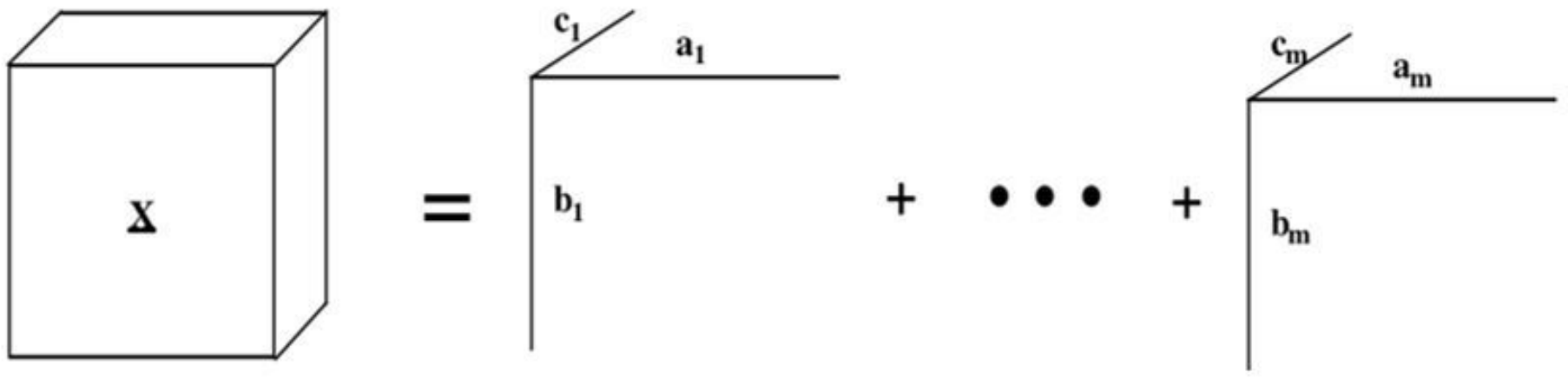

Figure 2

The general form of the parallel factorization model

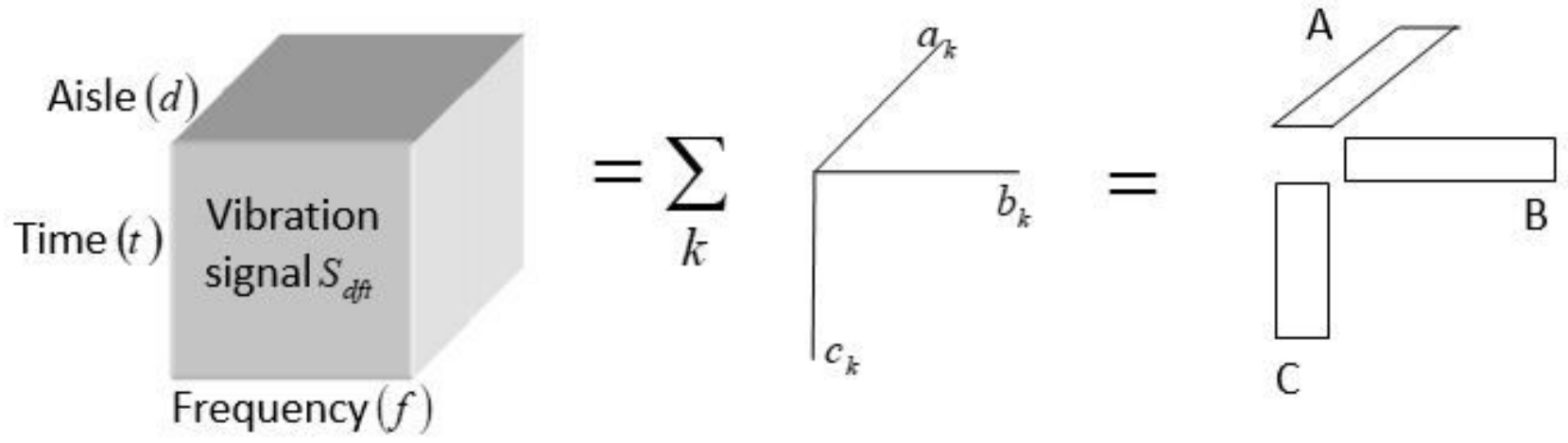

\section{Figure 3}

The model of multi-scale parallel factor decomposition for fault diagnosis 


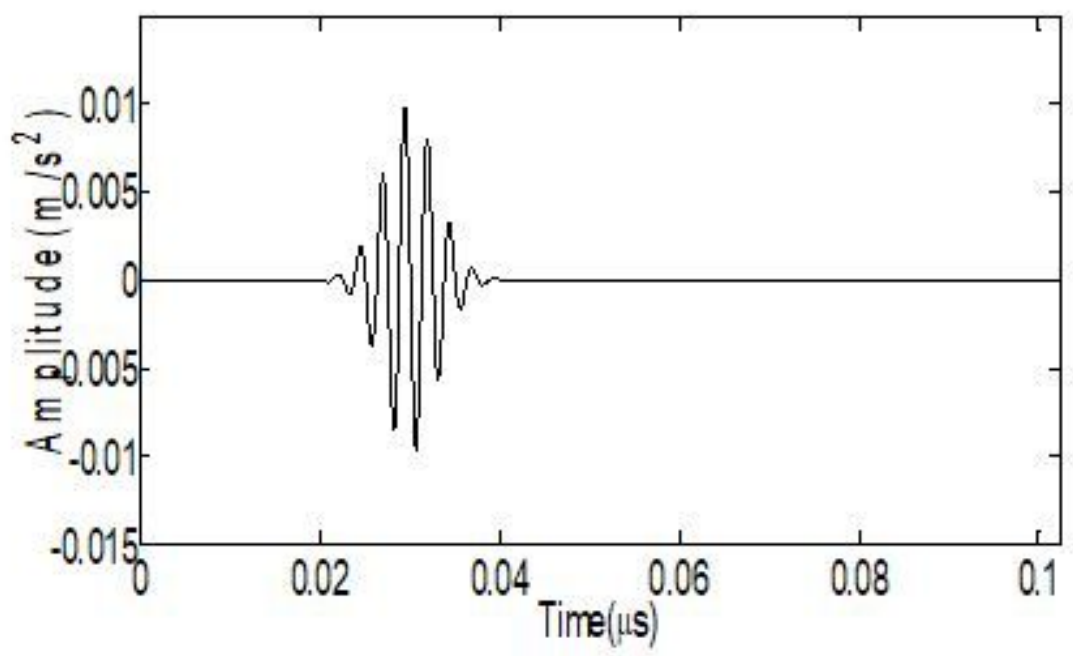

Figure 4

Time-domain diagram of the original simulated signal

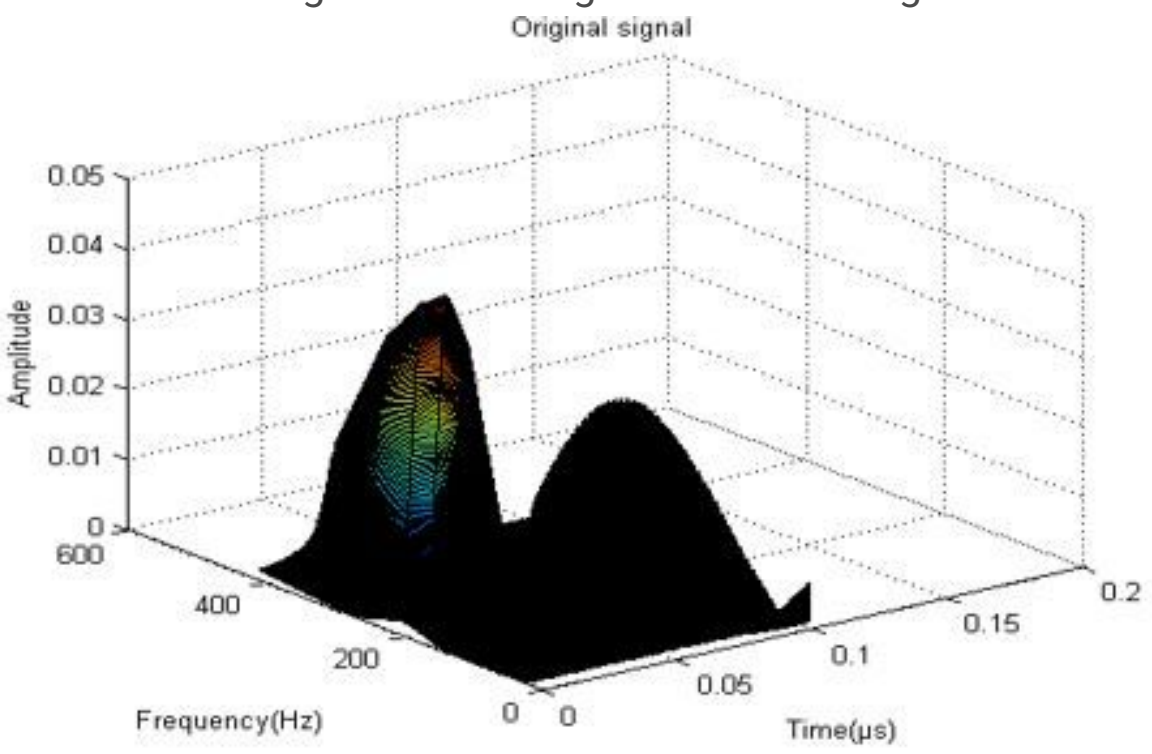

Figure 5

Time-frequency diagram of original simulation signal 

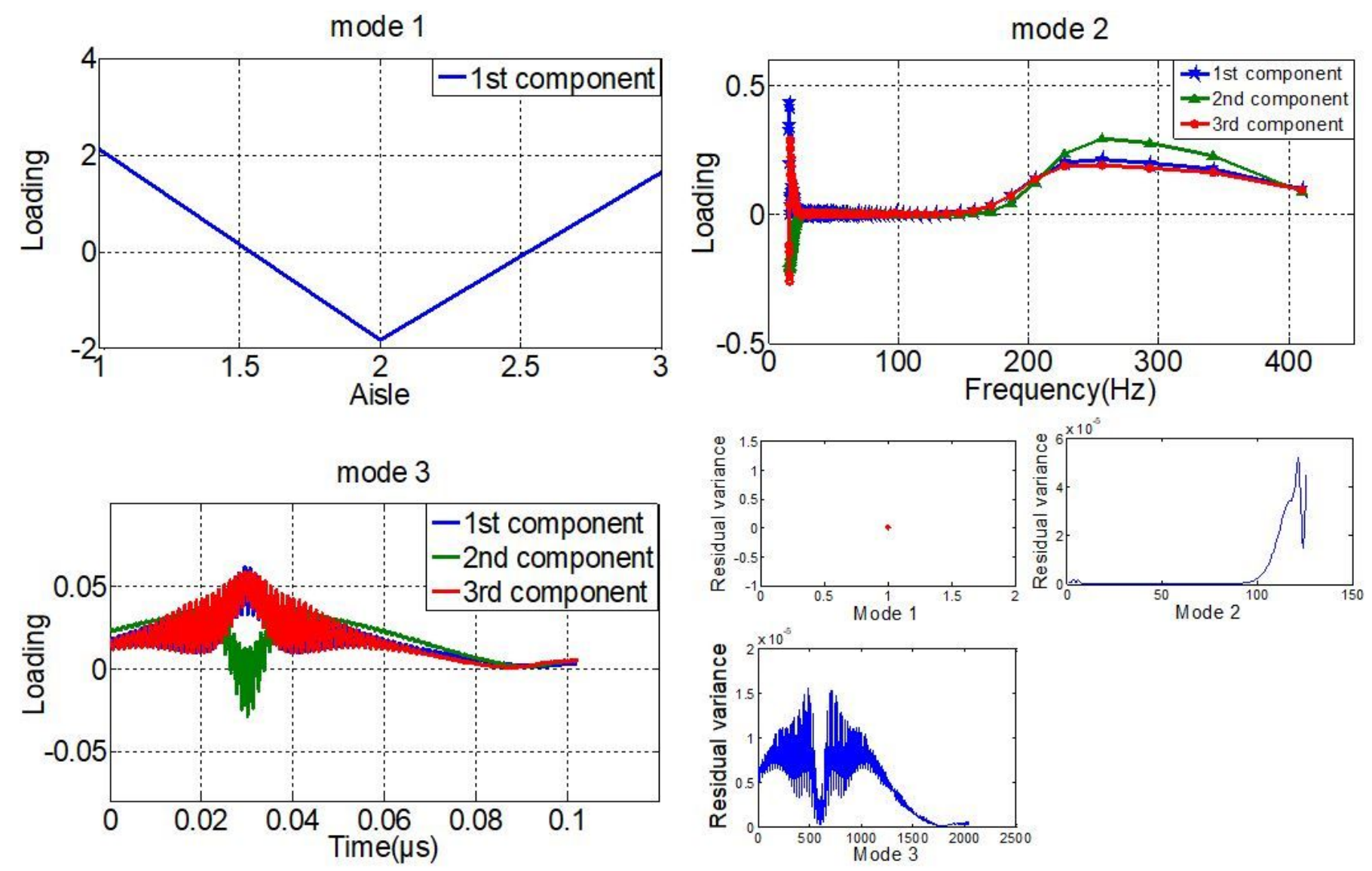

Figure 6

Parallel factor analysis of the original simulation signal

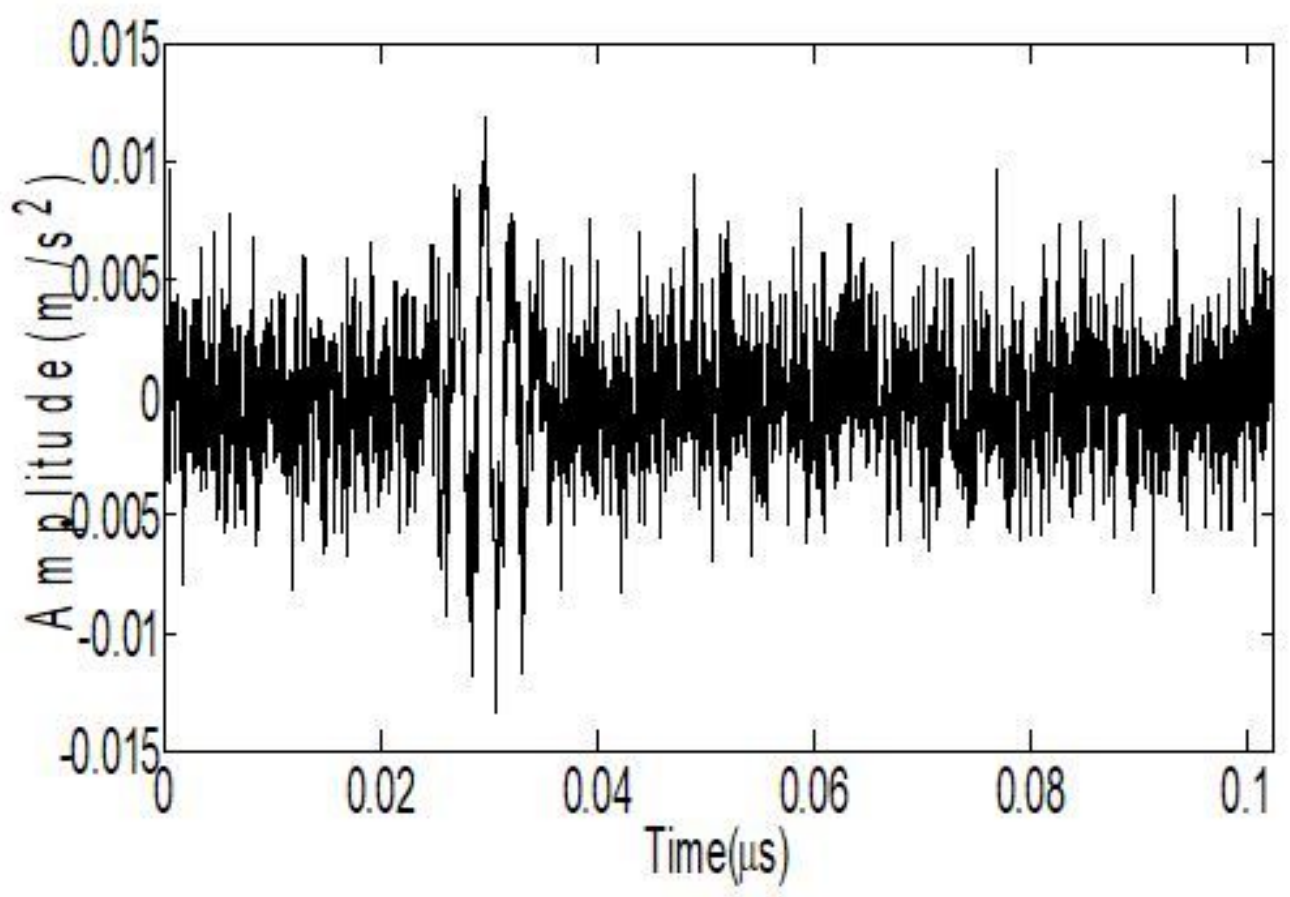

Figure 7 
Time-domain diagram of noise-added simulation signal

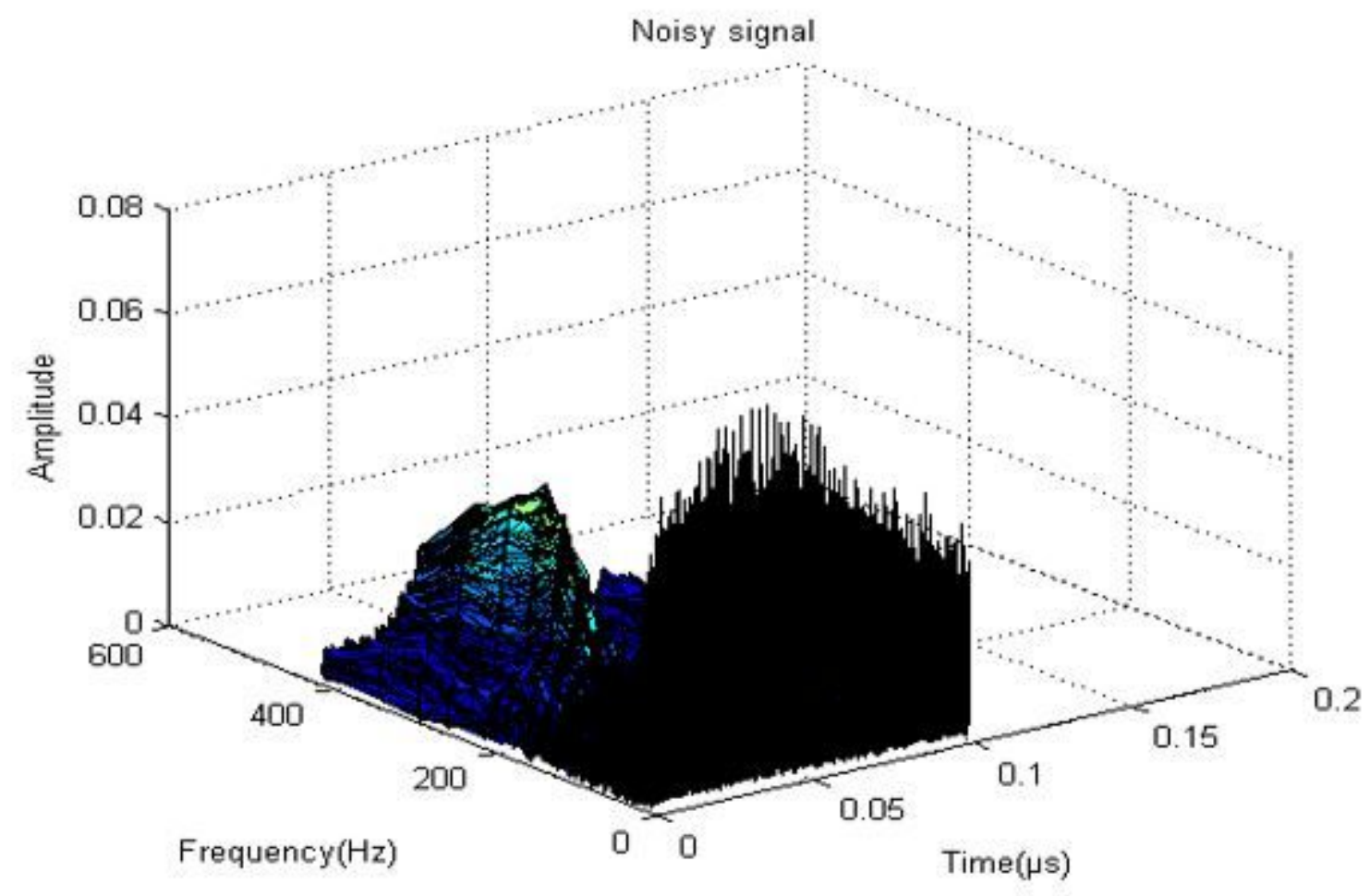

Figure 8

Time-frequency diagram of noise-added simulation signal 

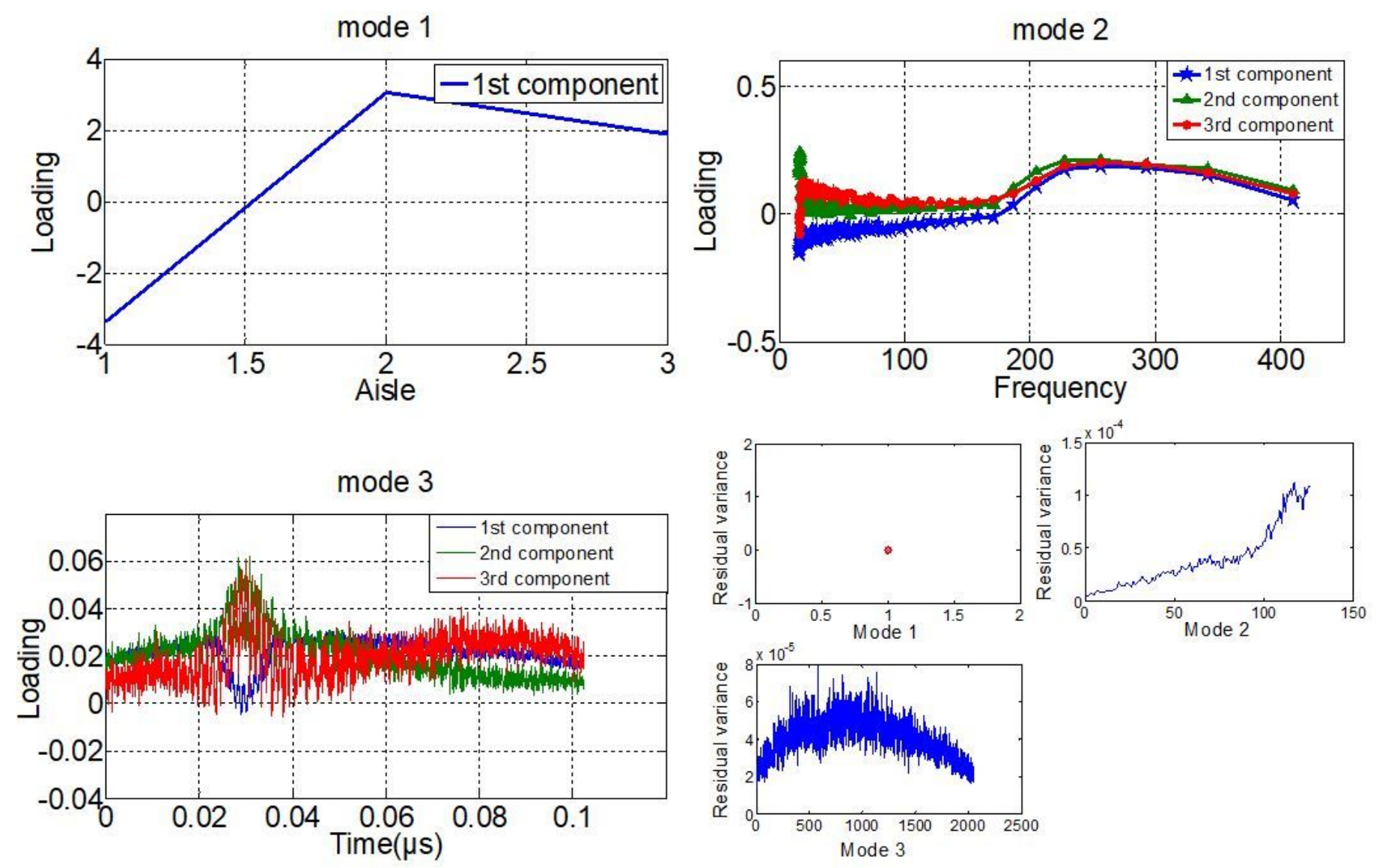

\section{Figure 9}

Parallel factor analysis of noise added simulation signal

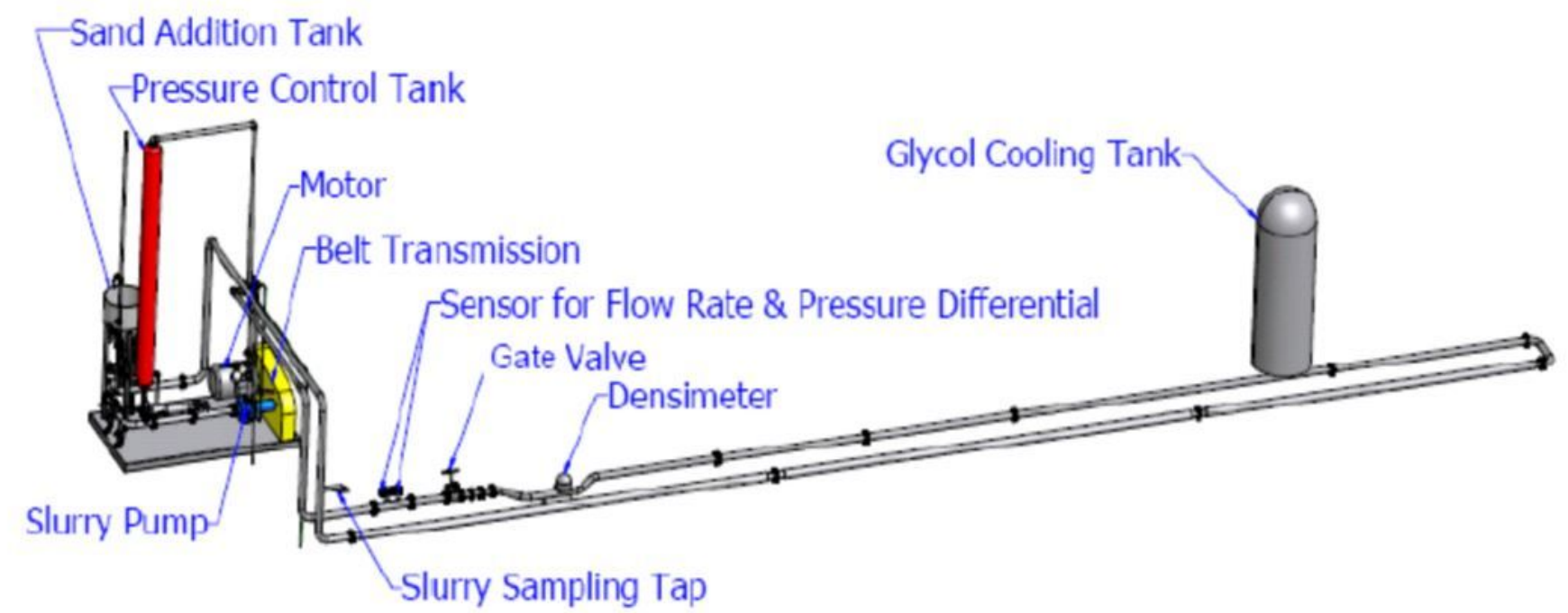

Figure 10

Schematic diagram of slurry pump fault diagnosis test system 


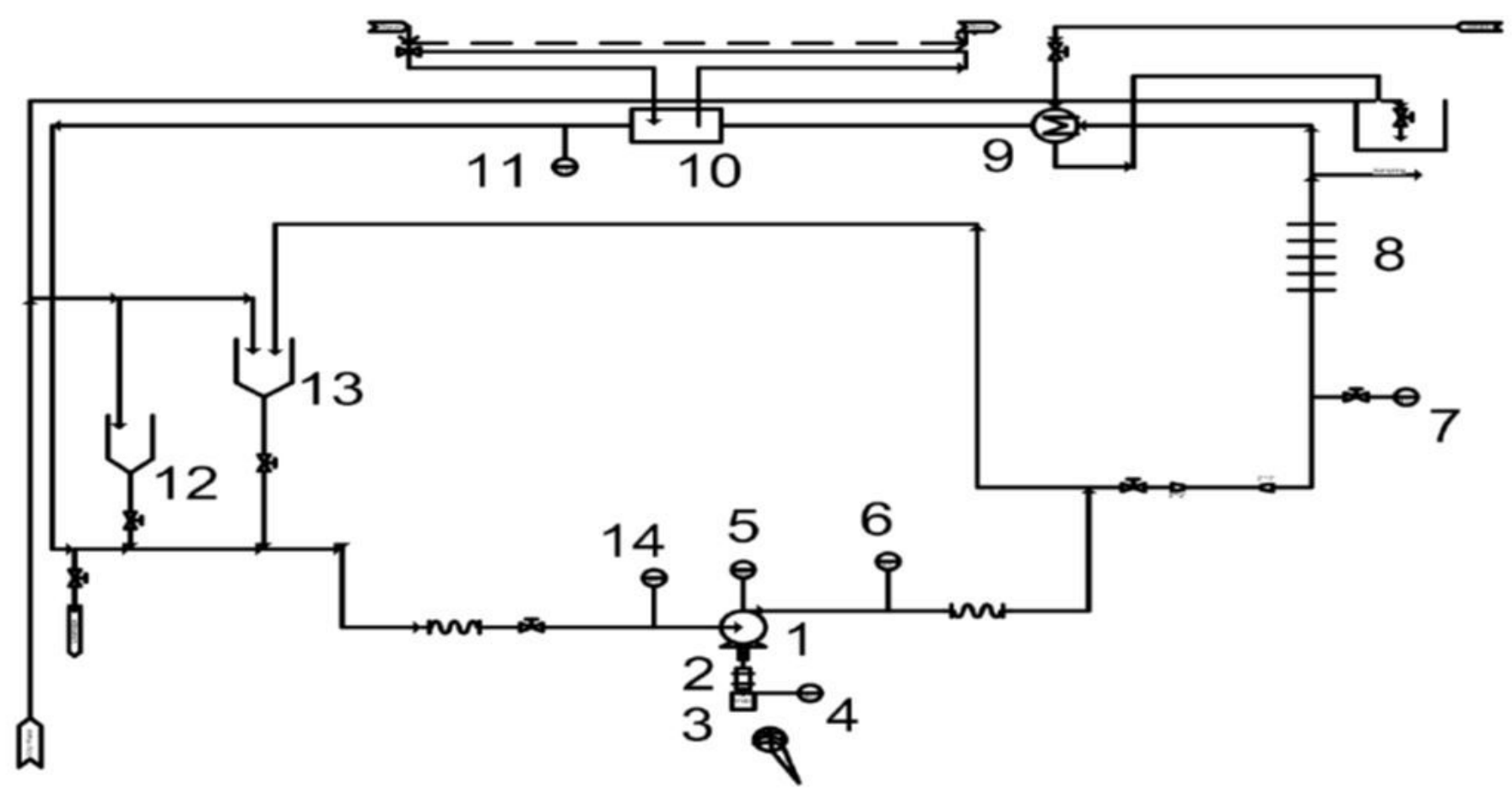

Figure 11

Process flow chart of fault diagnosis test system for slurry pump 


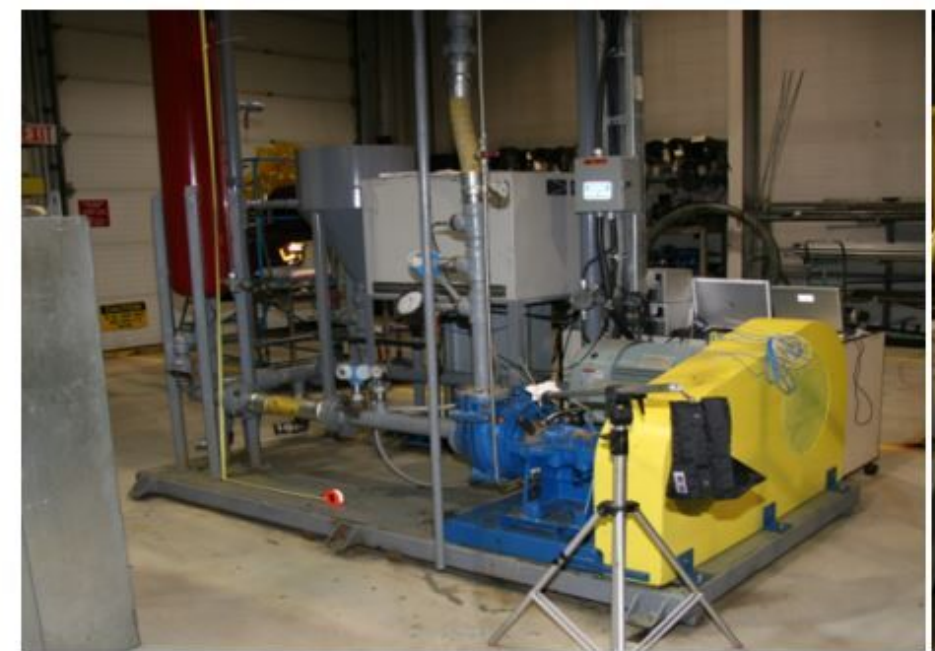

a. Centrifugal pump experimental equipment

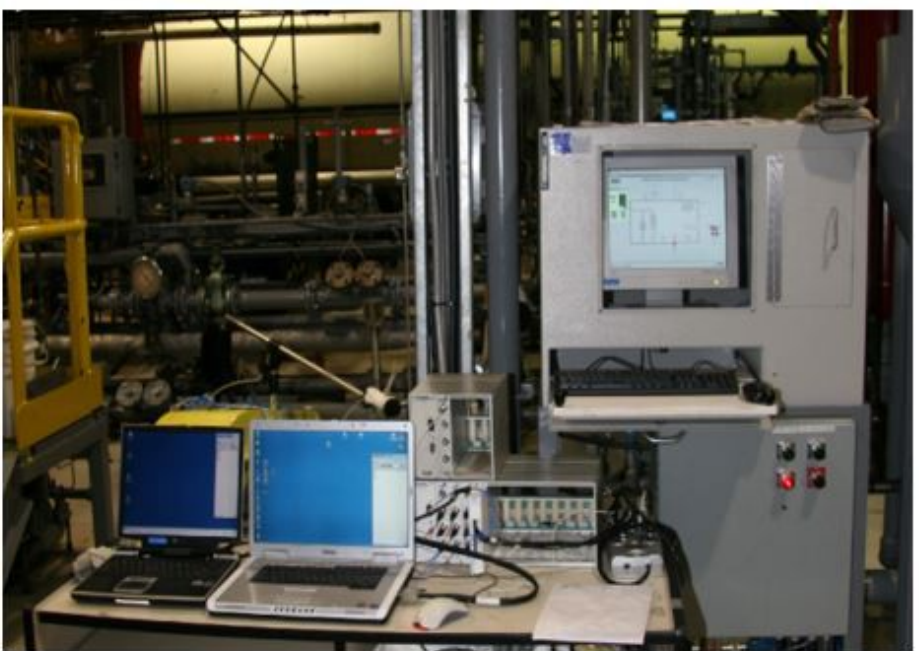

b. Signal acquisition system

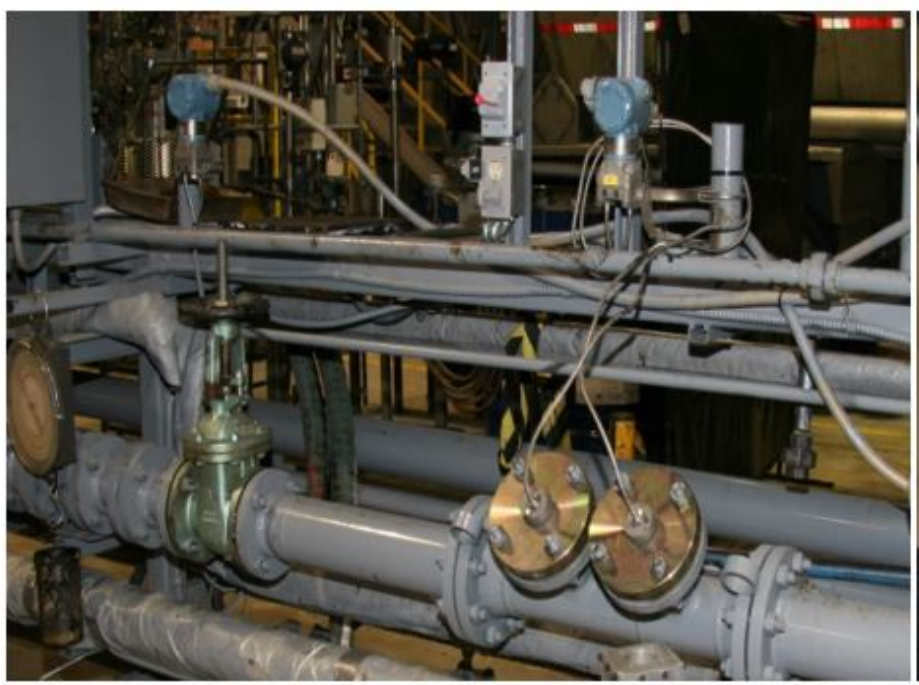

c. Sensor for flow rate

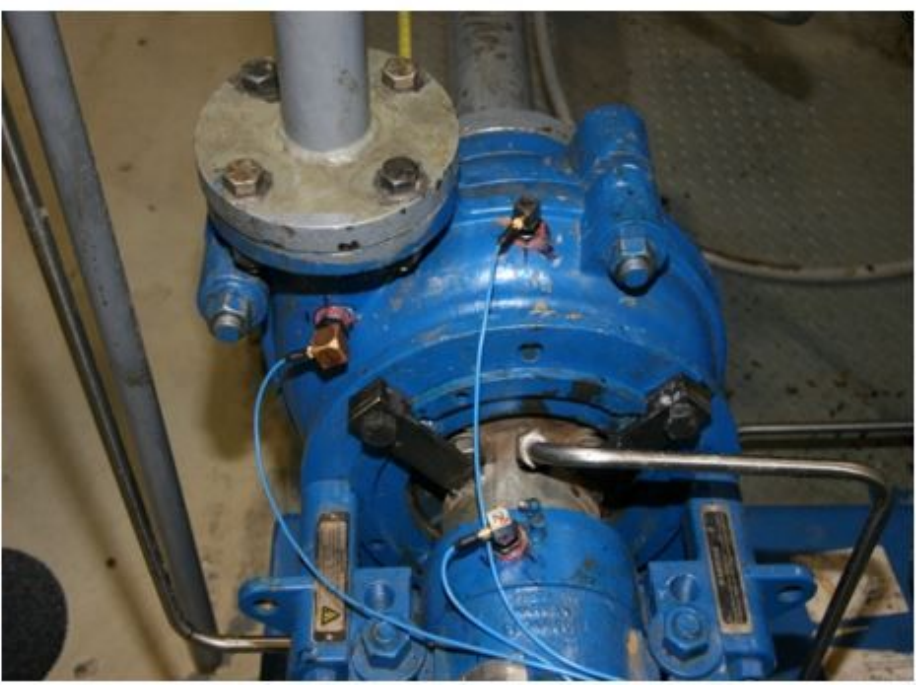

d. Installation diagram of acceleration sensor

Figure 12

Experiment system of slurry pump 


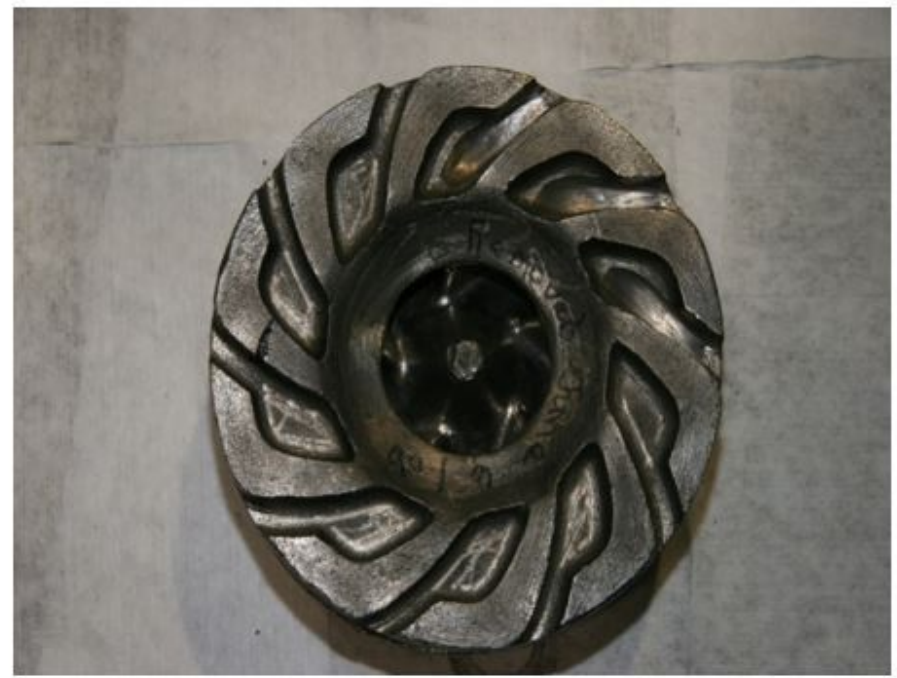

(a) S1 normal impeller

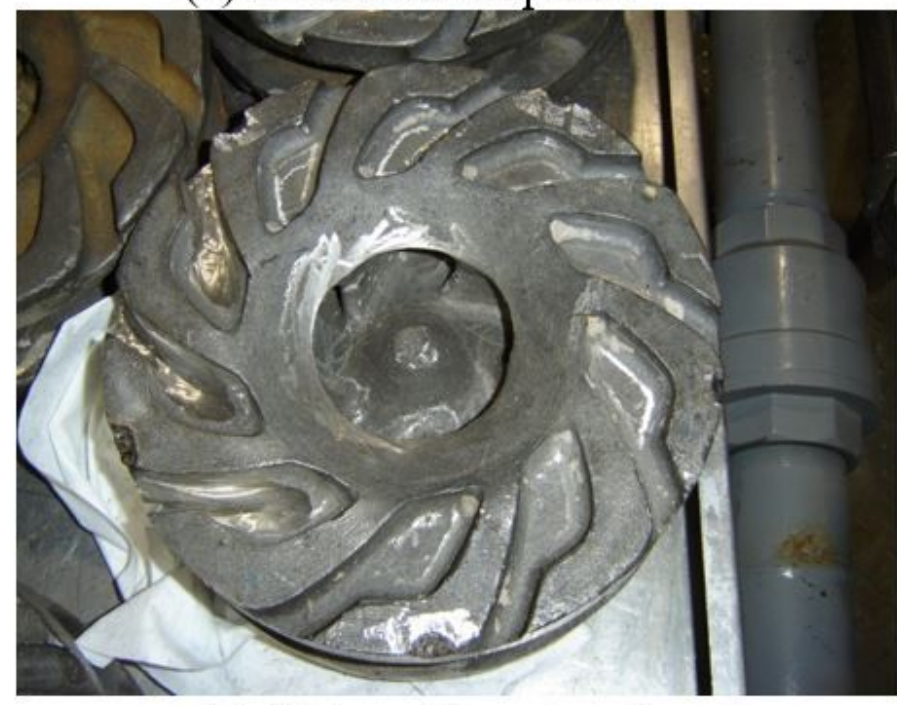

(c) S3 impeller edge damage

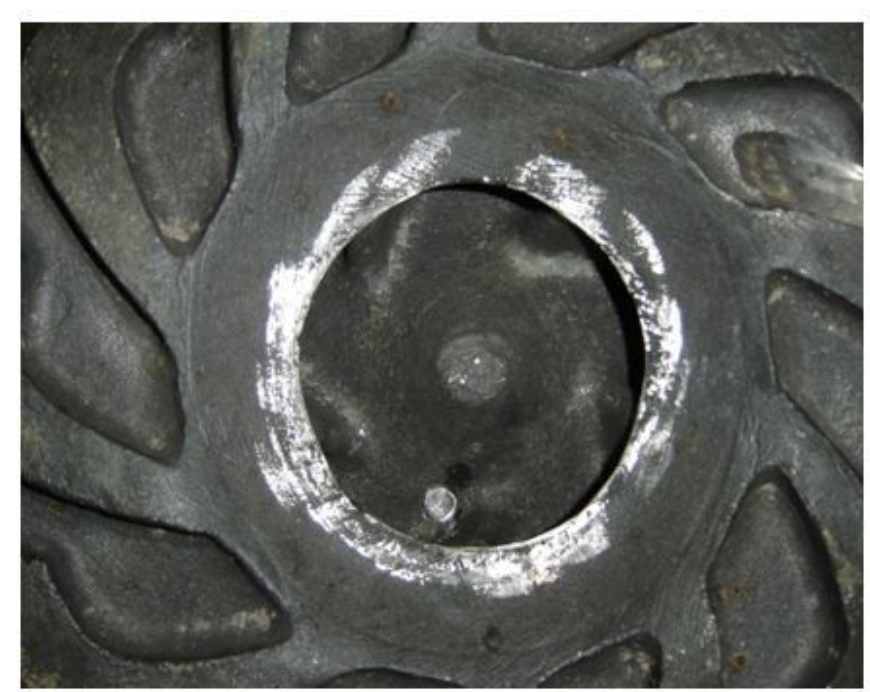

(b) S2 impeller perforation

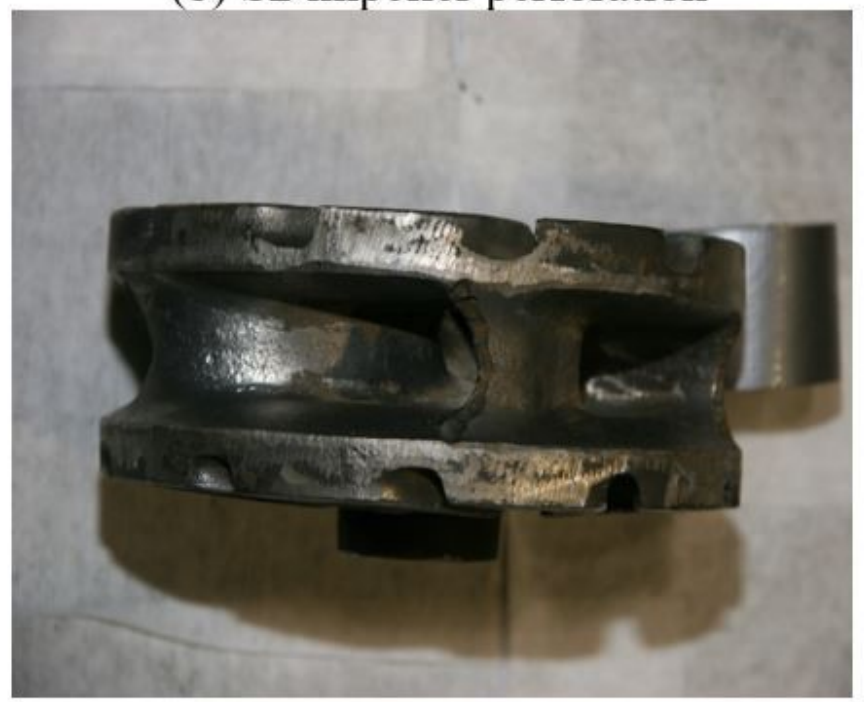

(d) S4 blade damage

Figure 13

Four failure modes of the impeller in the experiment 


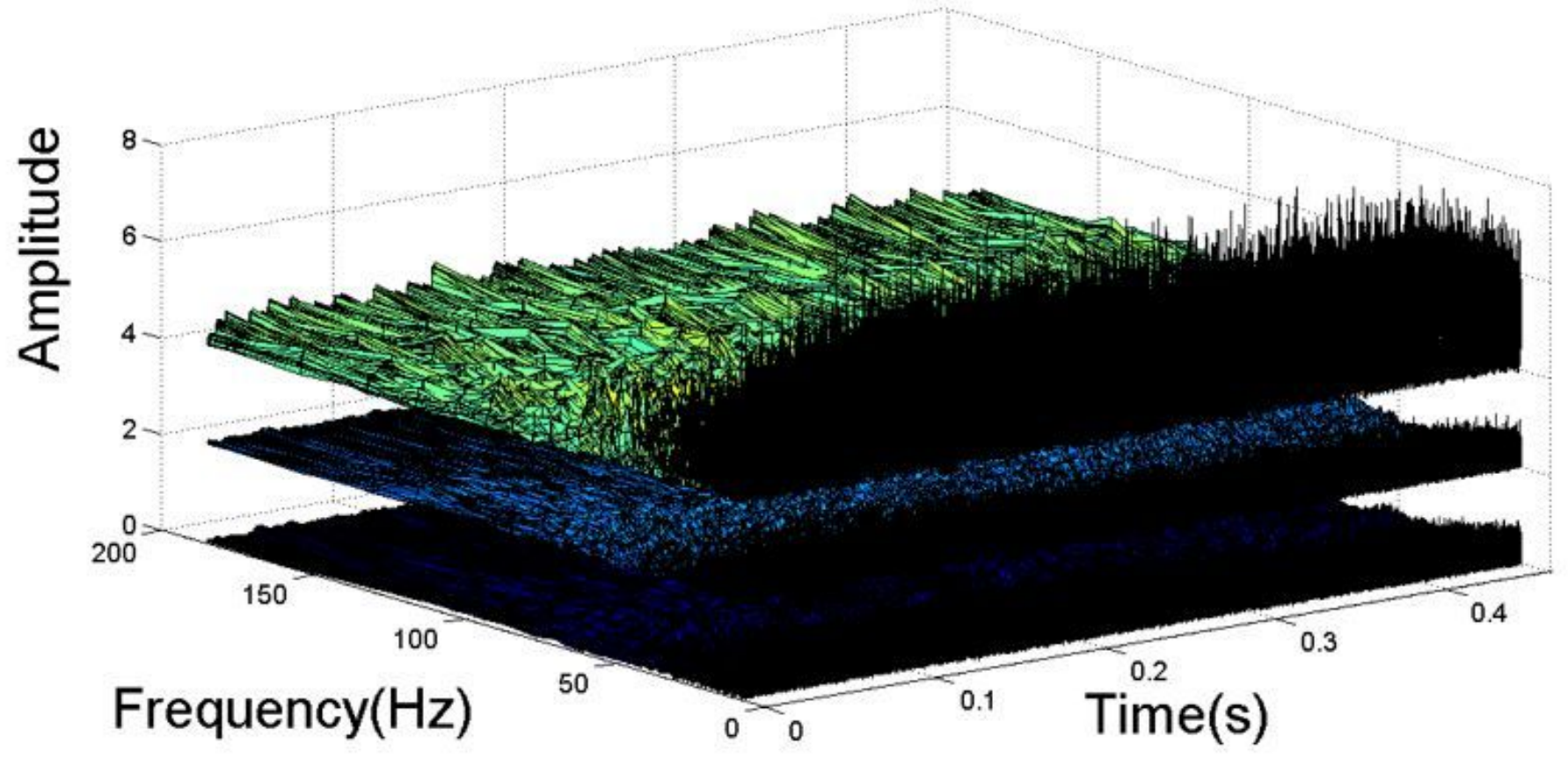

Figure 14

Time-frequency diagram of 3 vibration signals in normal state S1

mode 1

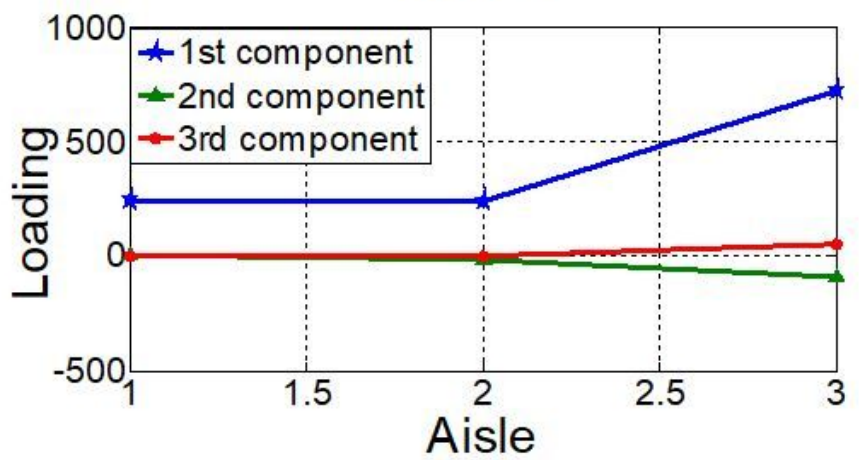

mode 3

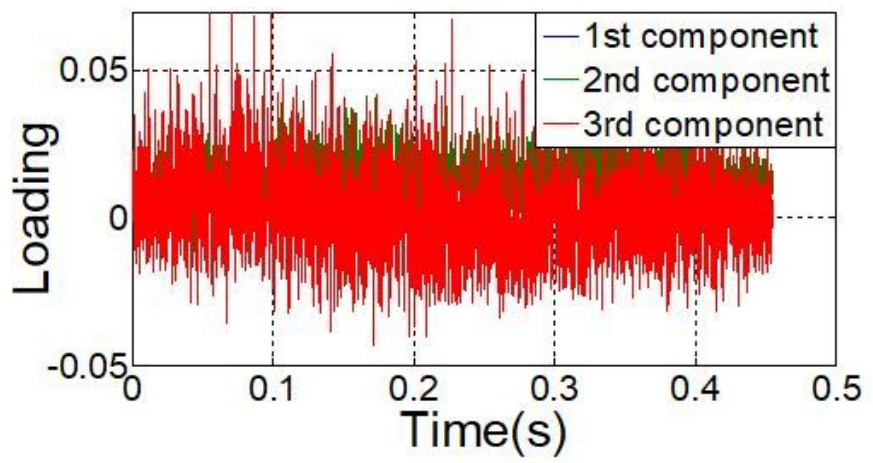

mode 2
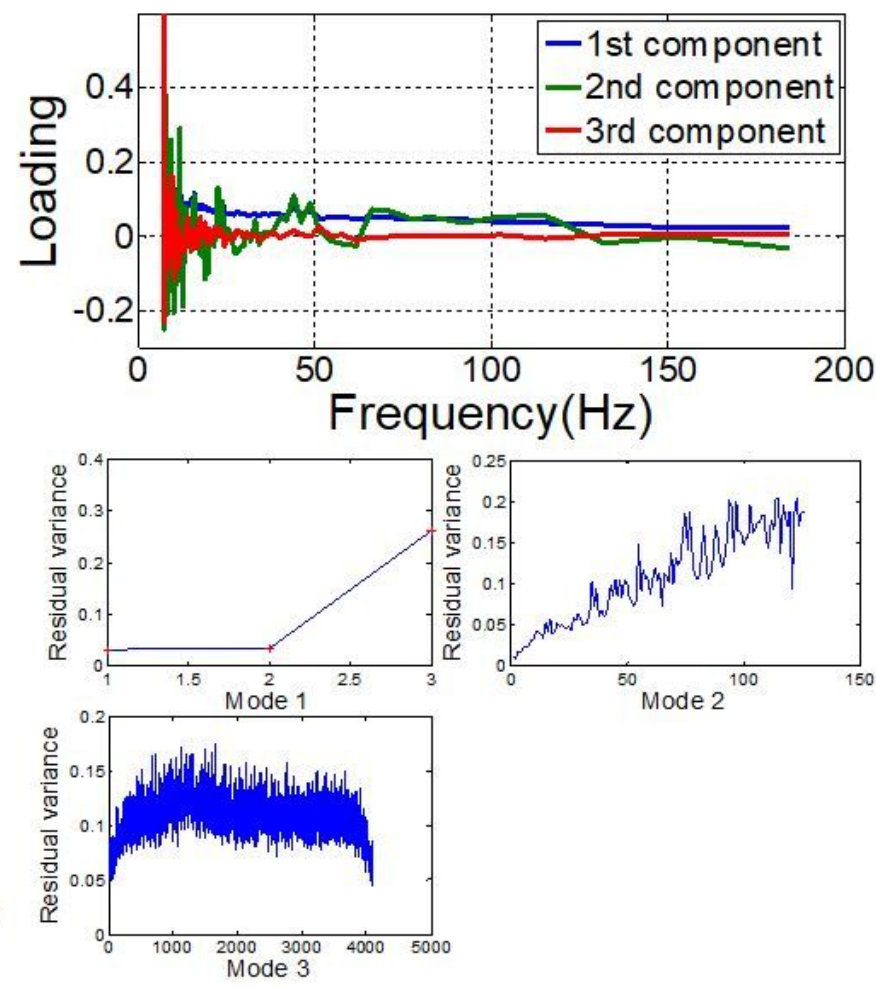

Figure 15

Parallel factor analysis when the centrifugal pump in normal state S1 


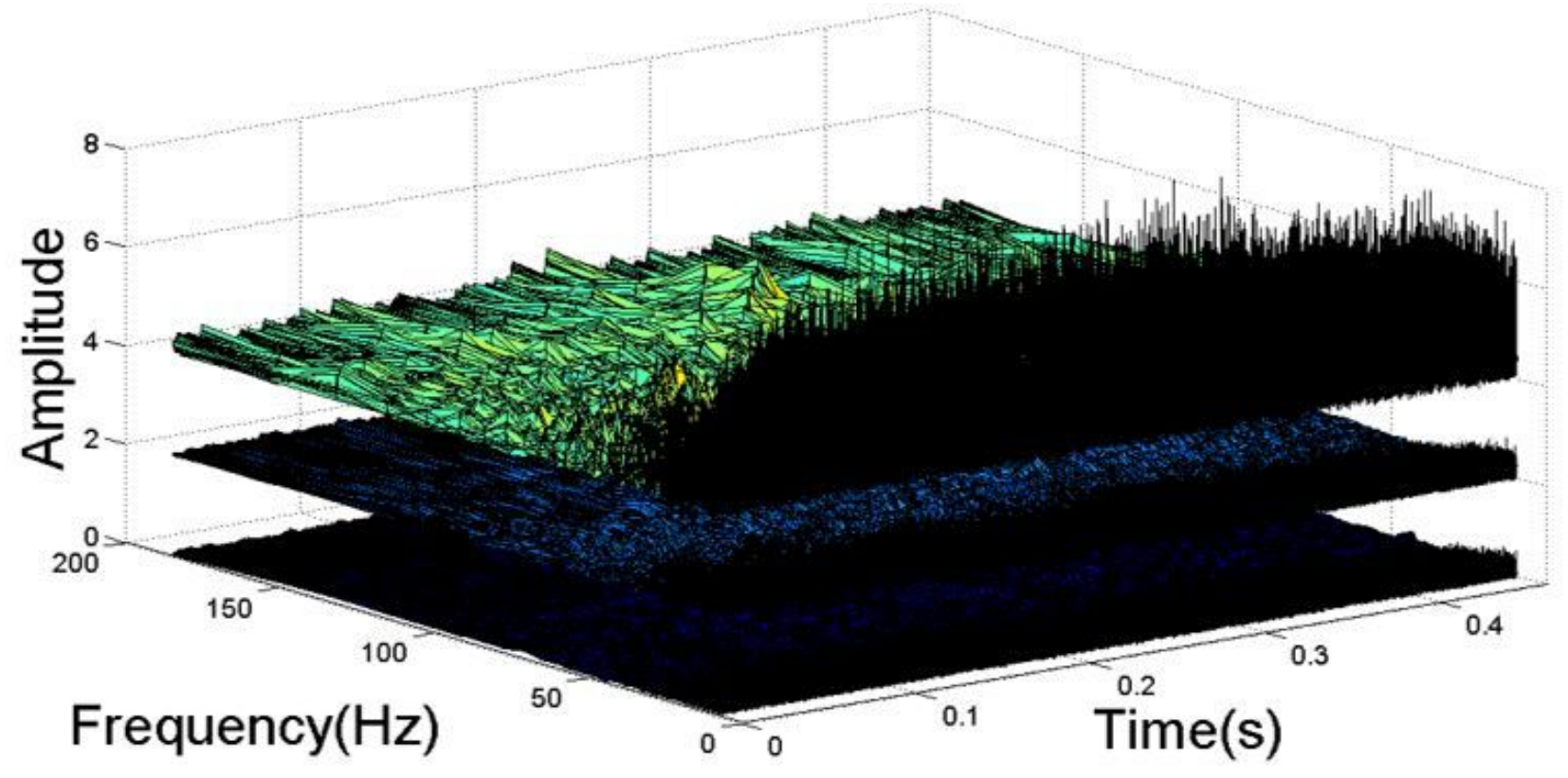

Figure 16

Time-frequency diagrams of 3 vibration signals under fault state S2

mode 1

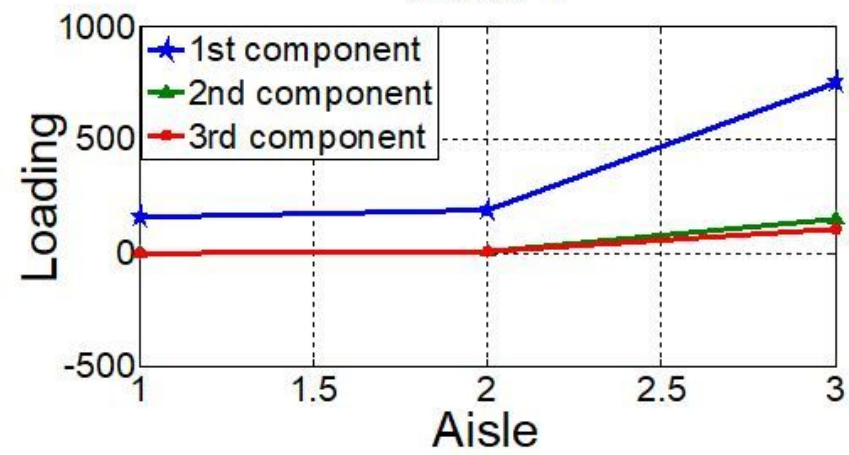

mode 3

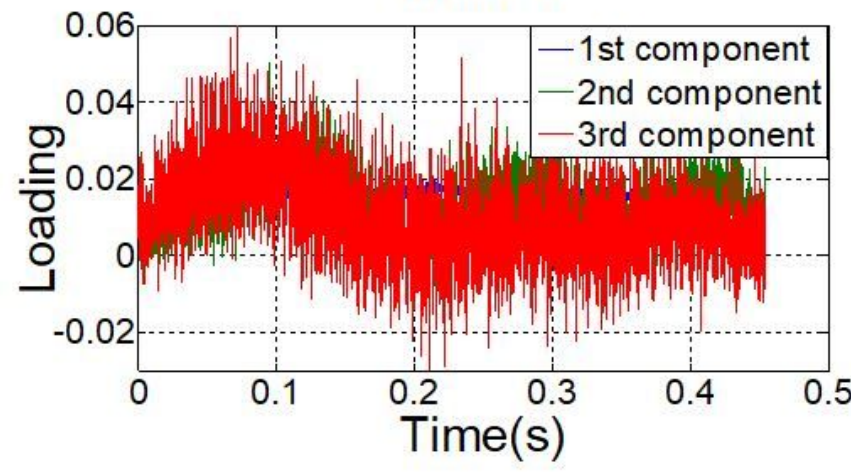

mode 2
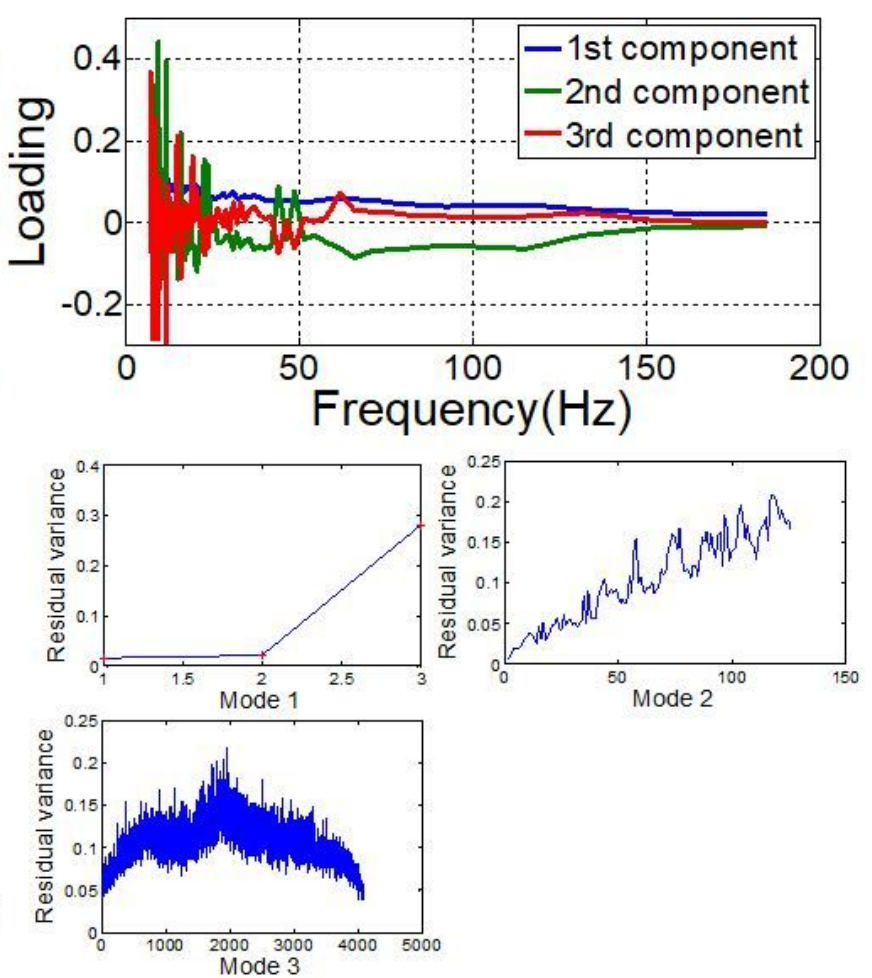

Figure 17

Parallel factor analysis when the centrifugal pump fails state $\mathrm{S} 2$ 


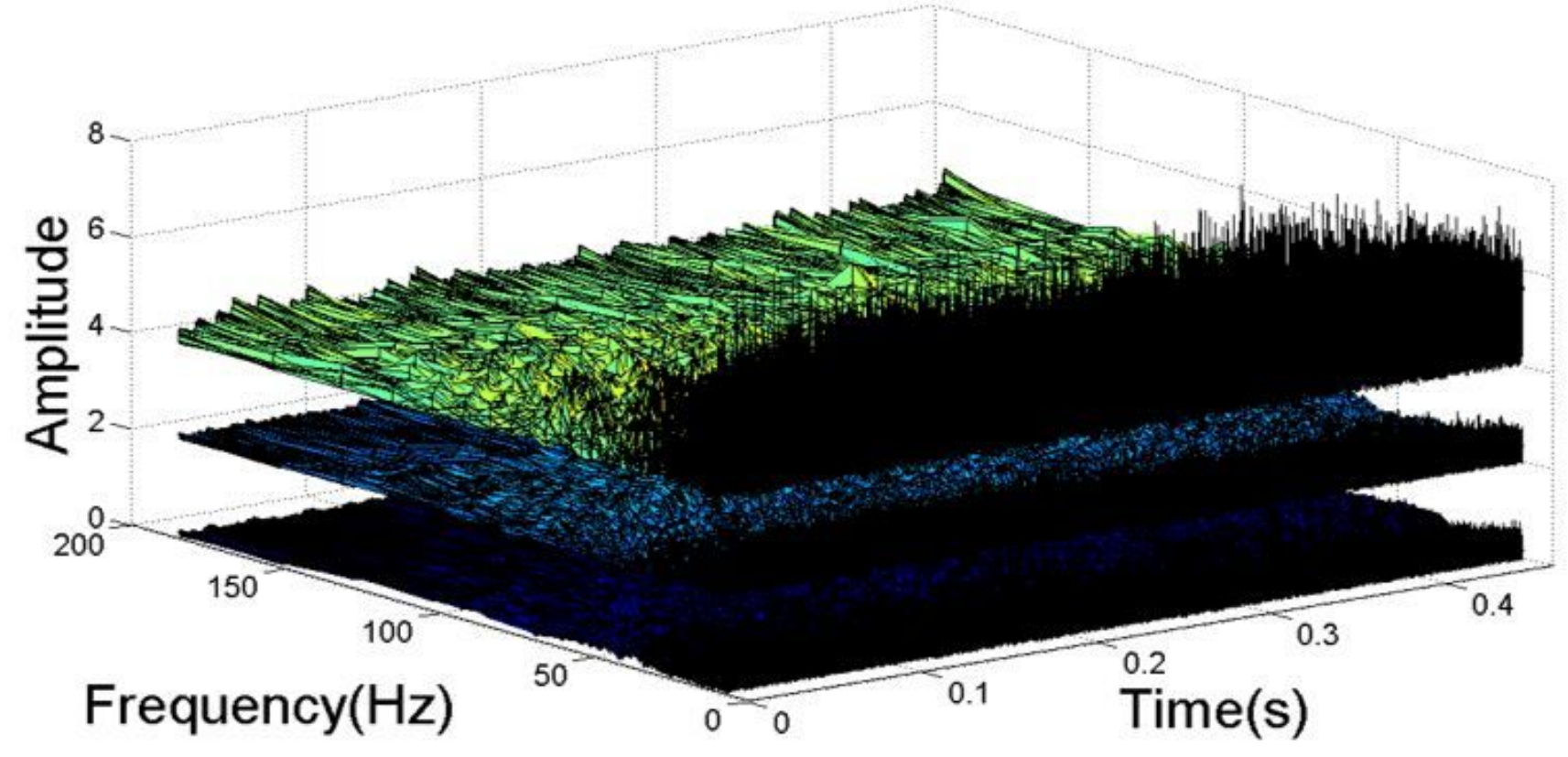

Figure 18

Time-frequency diagrams of 3 vibration signals under fault state $S 3$

mode 1

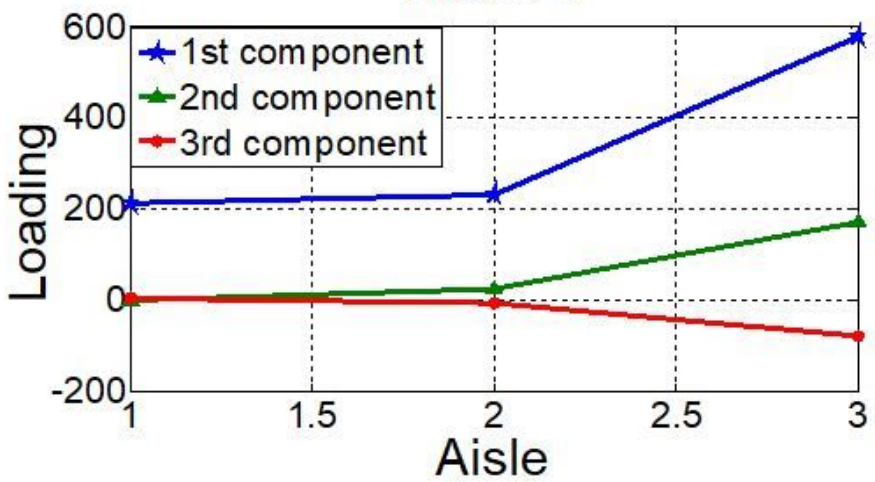

mode 3

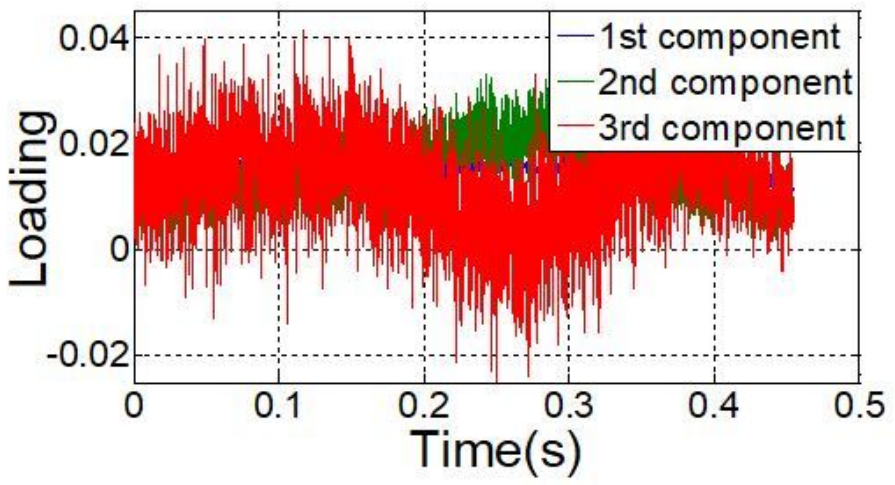

mode 2
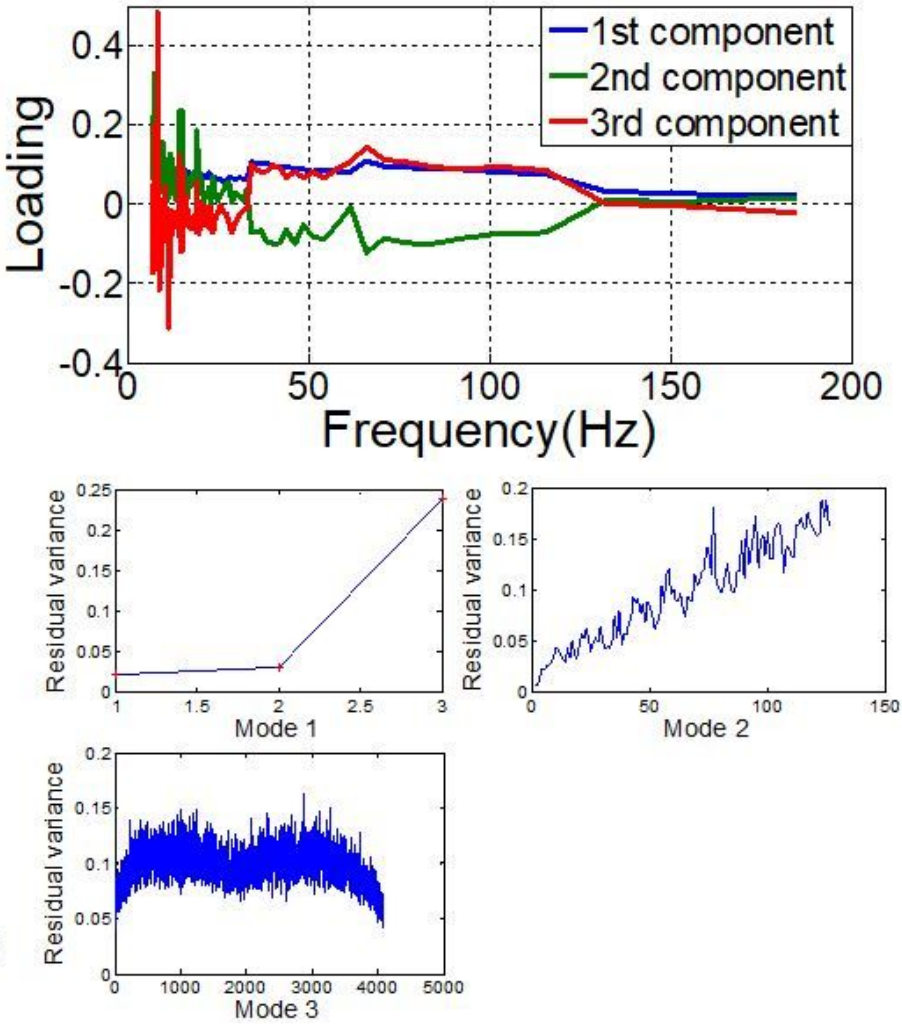

Figure 19

Parallel factor analysis when the centrifugal pump fails state S3 


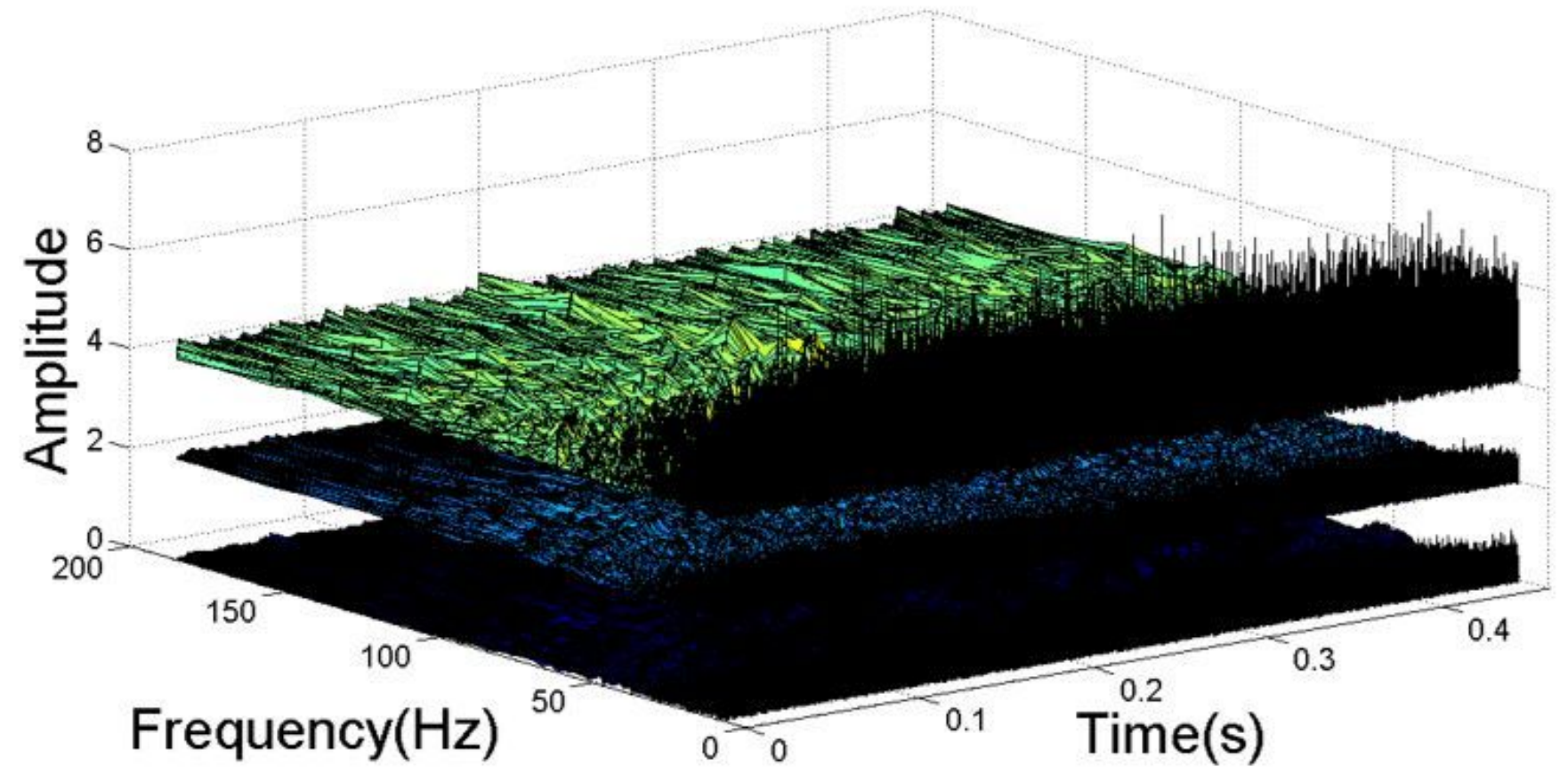

Figure 20

Time-frequency diagrams of 3 vibration signals under fault state $\mathrm{S} 4$
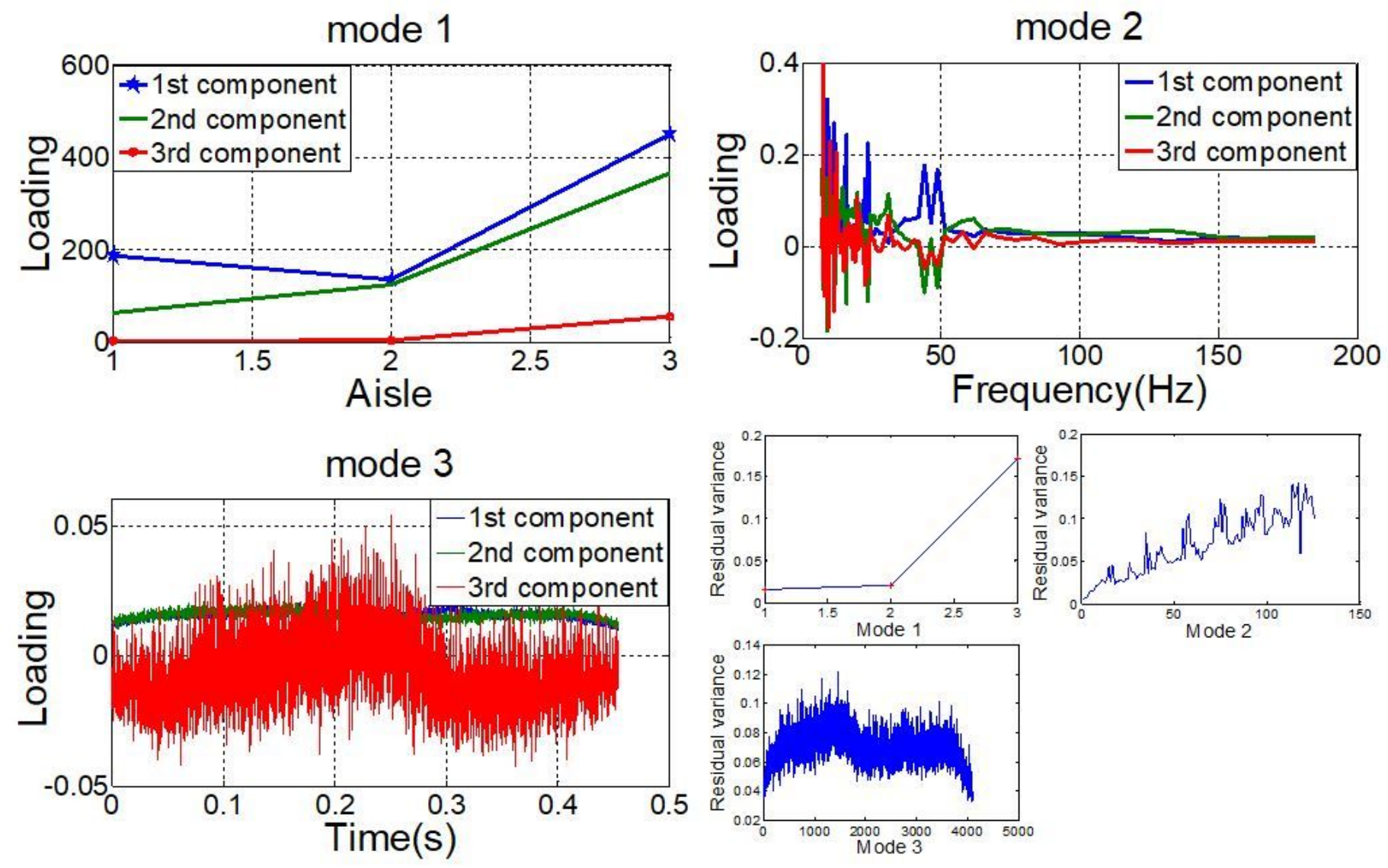

Figure 21 


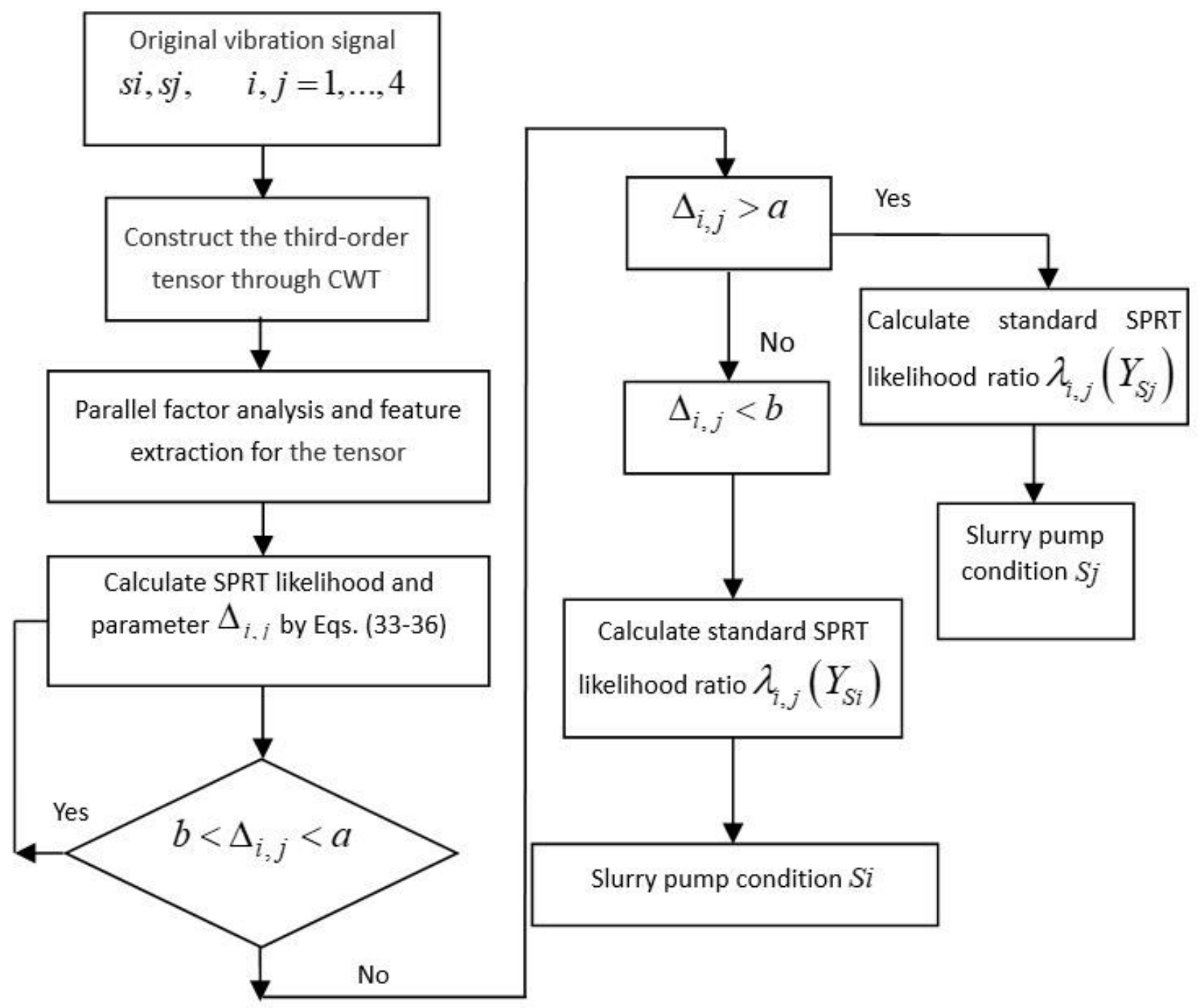

Figure 22

Diagnostic process based on binary SPRT for centrifugal pump 

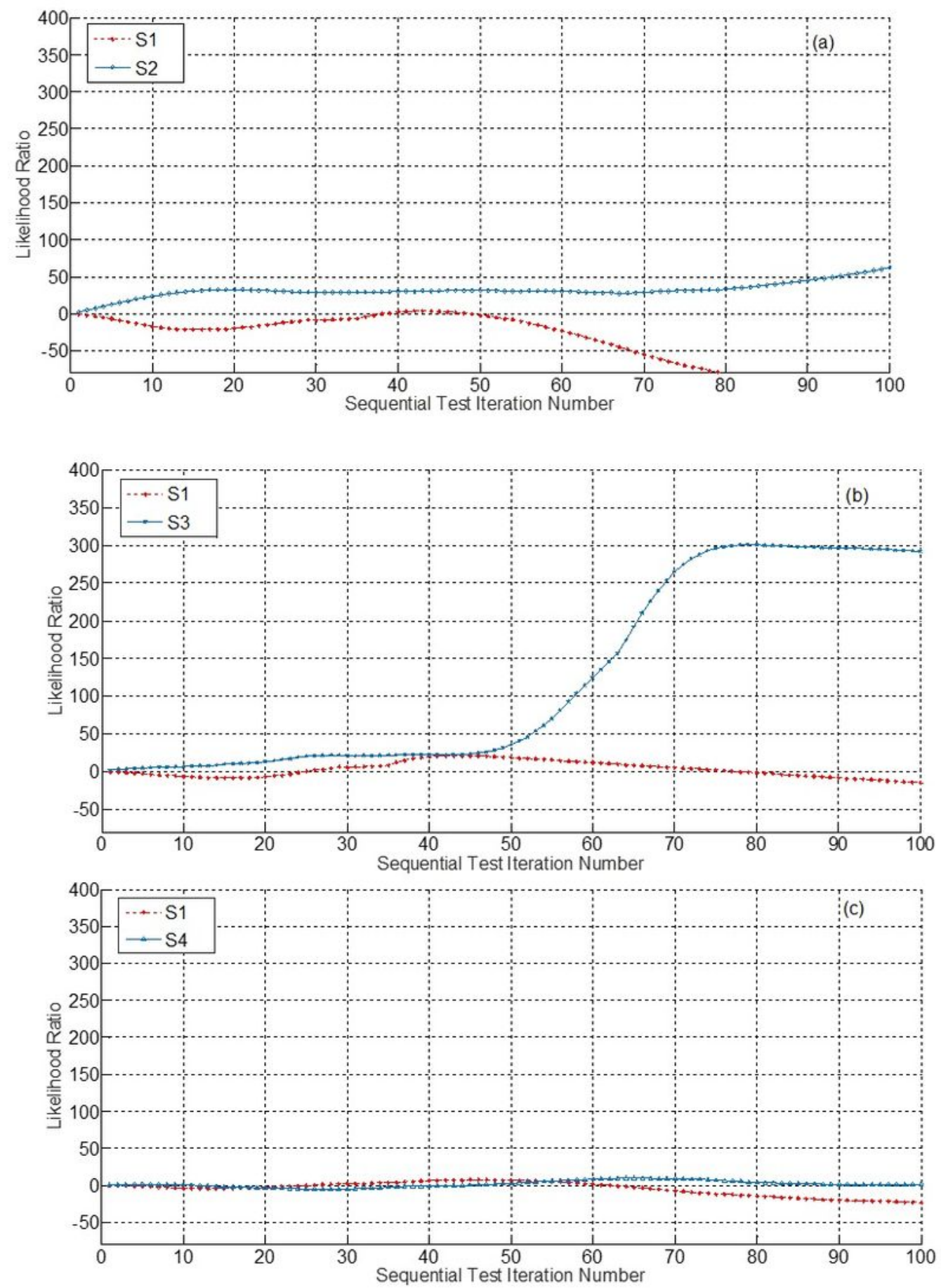

Figure 23

Result of SPRT in variables $\lambda_{-} \_1,2, \lambda_{-} 1,3, \lambda_{-} \_1,4$ with calculation iteration 

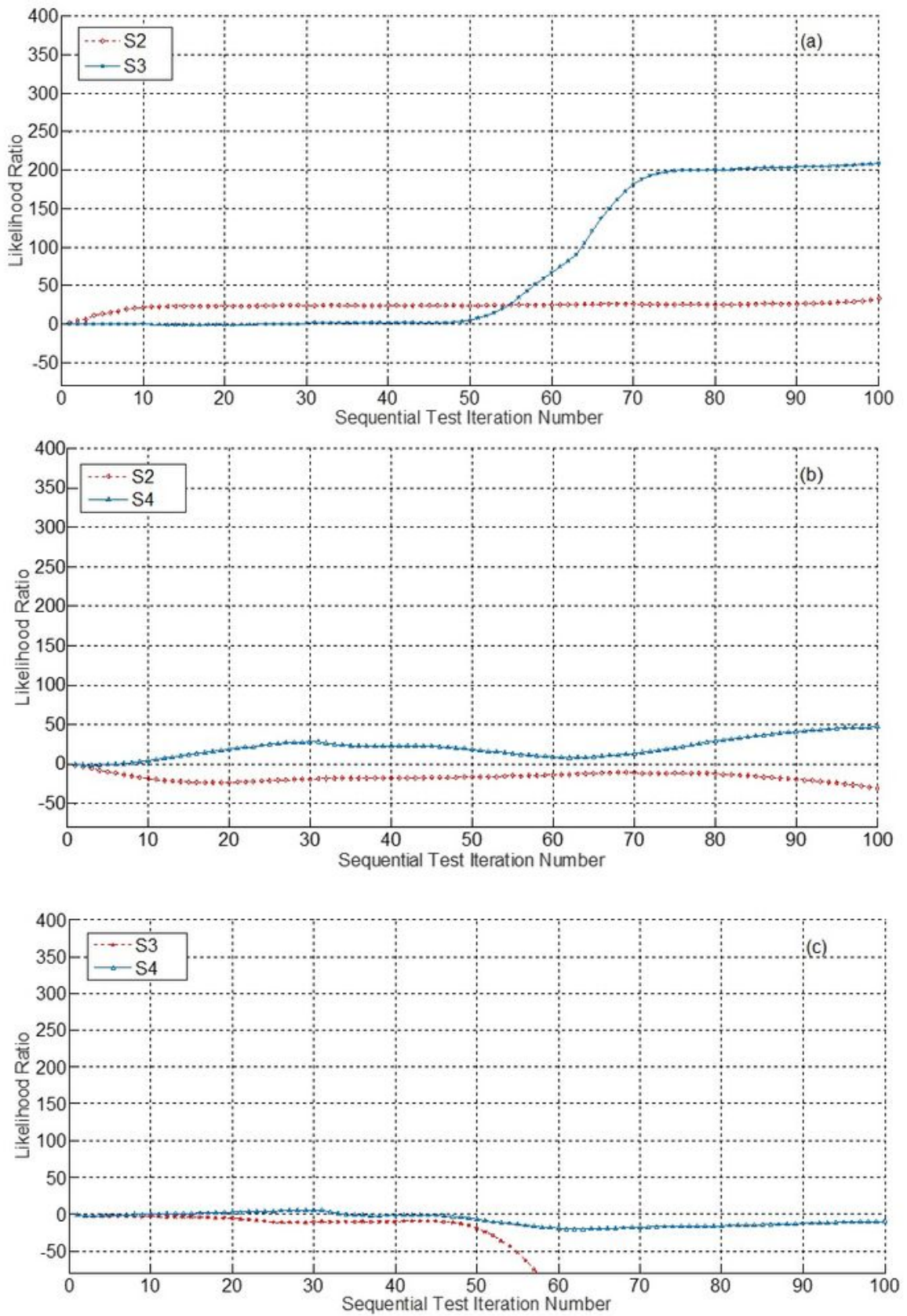

Figure 24

Result of SPRT in variables $\lambda \_2,3, \lambda \_2,4, \lambda \_3,4$ with calculation cycles 